Supporting Information

\title{
Lycibarbarspermidines A-O, New Dicaffeoylspermidine Derivatives from Wolfberry, with Activities against Alzheimer's Disease and Oxidation
}

Zheng-Qun Zhou ${ }^{\dagger, \#}$, Hong-Xia Fan ${ }^{\dagger, \#}$, Rong-Rong $\mathrm{He}^{\dagger}$, Jia Xiao ${ }^{\S}$, Bun Tsoi ${ }^{\dagger}$, Kang-Hua Lan ${ }^{\dagger}$, Hiroshi Kurihara ${ }^{\dagger}$, Kwok-Fai So ${ }^{\ddagger}$, Xin-Sheng Yao ${ }^{\dagger, *}$, Hao Gao ${ }^{\dagger, *}$

${ }^{\dagger}$ Institute of Traditional Chinese Medicine and Natural Products, College of Pharmacy, Jinan University, Guangzhou 510632, People's Republic of China

${ }^{\S}$ Department of Immunobiology, Institute of Tissue Transplantation and Immunology, College of Life Science and Technology, Jinan University, Guangzhou 510632, People's Republic of China

Guangdong Medical Key Laboratory of Brain Function and Diseases, GMH Institute of Central Nervous System Regeneration, Jinan University, Guangzhou 510632, People's Republic of China

\#These authors contributed equally to this work

*Corresponding Authors. Phone/fax: +86-20-85221559. E-mail: tghao@jnu.edu.cn (H. Gao); tyaoxs@jnu.edu.cn (X.-S. Yao). 


\section{List of Supporting Information}

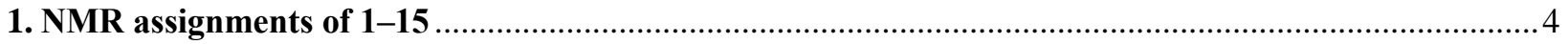

Table S1. NMR (600 MHz for ${ }^{1} \mathrm{H}$ NMR, $150 \mathrm{MHz}$ for ${ }^{13} \mathrm{C}$ NMR, DMSO- $d_{6}$ ) assignments of $\mathbf{1} \ldots \ldots \ldots \ldots \ldots . . . . . . . .4$

Table S2. NMR ( $600 \mathrm{MHz}$ for ${ }^{1} \mathrm{H}$ NMR, $150 \mathrm{MHz}$ for ${ }^{13} \mathrm{C}$ NMR, DMSO- $d_{6}$ ) assignments of 2................. 6

Table S3. NMR (600 MHz for ${ }^{1} \mathrm{H}$ NMR, $150 \mathrm{MHz}$ for ${ }^{13} \mathrm{C}$ NMR, DMSO- $d_{6}$ ) assignments of 3................. 8

Table S4. NMR (600 MHz for ${ }^{1} \mathrm{H}$ NMR, $150 \mathrm{MHz}$ for ${ }^{13} \mathrm{C}$ NMR, DMSO- $\left.d_{6}\right)$ assignments of 4............... 10

Table S5. NMR (600 MHz for ${ }^{1} \mathrm{H}$ NMR, $150 \mathrm{MHz}$ for ${ }^{13} \mathrm{C}$ NMR, DMSO- $d_{6}$ ) assignments of 5................ 12

Table S6. NMR (600 MHz for ${ }^{1} \mathrm{H}$ NMR, $150 \mathrm{MHz}$ for ${ }^{13} \mathrm{C}$ NMR, DMSO- $d_{6}$ ) assignments of $6 \ldots \ldots \ldots \ldots \ldots . . . . . . .14$

Table S7. NMR (300 MHz for ${ }^{1} \mathrm{H}$ NMR, $75 \mathrm{MHz}$ for ${ }^{13} \mathrm{C}$ NMR, DMSO- $d_{6}$ ) assignments of 7.................. 16

Table S8. NMR (600 MHz for ${ }^{1} \mathrm{H}$ NMR, $150 \mathrm{MHz}$ for ${ }^{13} \mathrm{C}$ NMR, DMSO- $d_{6}$ ) assignments of 8................ 18

Table S9. NMR (600 MHz for ${ }^{1} \mathrm{H}$ NMR, $150 \mathrm{MHz}$ for ${ }^{13} \mathrm{C}$ NMR, DMSO- $d_{6}$ ) assignments of 9................20

Table S10. NMR (300 MHz for ${ }^{1} \mathrm{H}$ NMR, $75 \mathrm{MHz}$ for ${ }^{13} \mathrm{C}$ NMR, $\mathrm{CD}_{3} \mathrm{OD}$ ) assignments of 10..................22

Table S11. NMR (600 MHz for ${ }^{1} \mathrm{H}$ NMR, $100 \mathrm{MHz}$ for ${ }^{13} \mathrm{C}$ NMR, DMSO- $d_{6}$ ) assignments of 11..............24

Table S12. NMR (600 MHz for ${ }^{1} \mathrm{H}$ NMR, $100 \mathrm{MHz}$ for ${ }^{13} \mathrm{C}$ NMR, DMSO- $d_{6}$ ) assignments of 12.............26

Table S13. NMR (600 MHz for ${ }^{1} \mathrm{H}$ NMR, $150 \mathrm{MHz}$ for ${ }^{13} \mathrm{C}$ NMR, DMSO- $d_{6}$ ) assignments of 13............28

Table S14. NMR (600 MHz for ${ }^{1} \mathrm{H}$ NMR, $150 \mathrm{MHz}$ for ${ }^{13} \mathrm{C}$ NMR, DMSO- $d_{6}$ ) assignments of 14.............30

Table S15. NMR (600 MHz for ${ }^{1} \mathrm{H}$ NMR, $150 \mathrm{MHz}$ for ${ }^{13} \mathrm{C}$ NMR, DMSO- $d_{6}$ ) assignments of 15............. 32

2. The result of short-term memory assay on the transgenic fly AD model of 1-15 .............................. 34

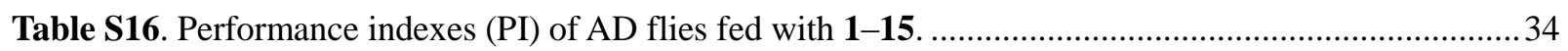

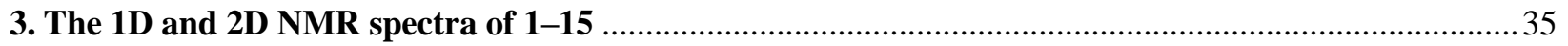

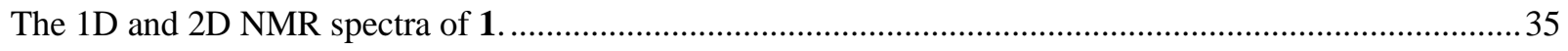

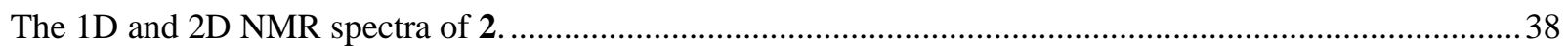

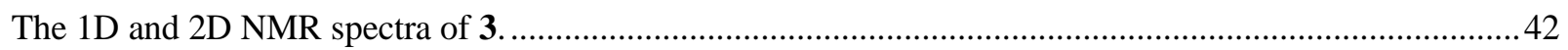

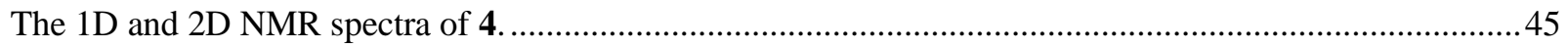

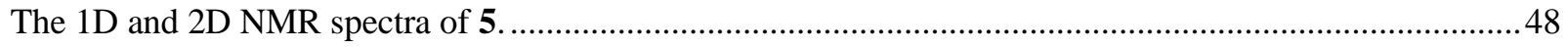

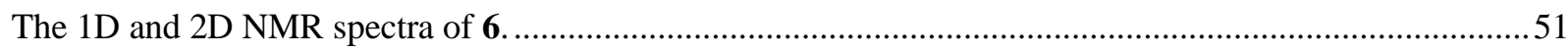

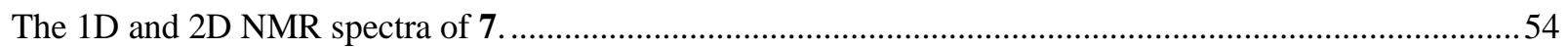




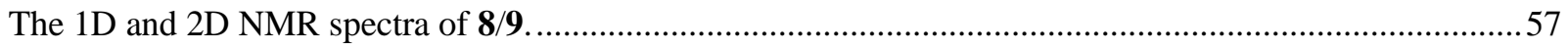

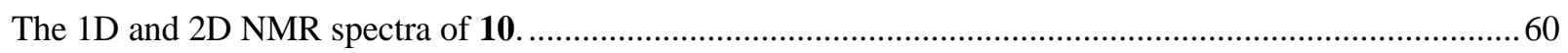

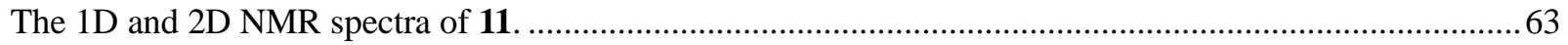

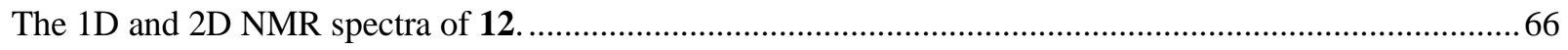

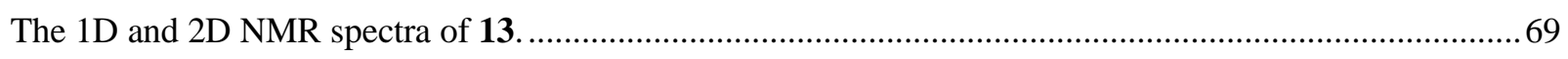

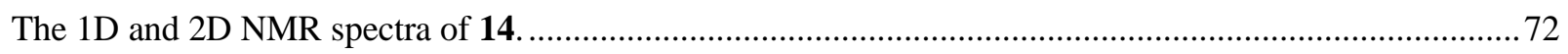

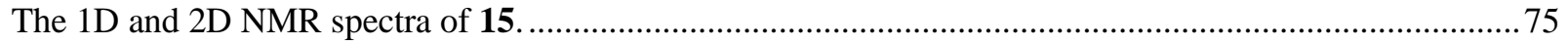




\section{NMR assignments of $1-15$}

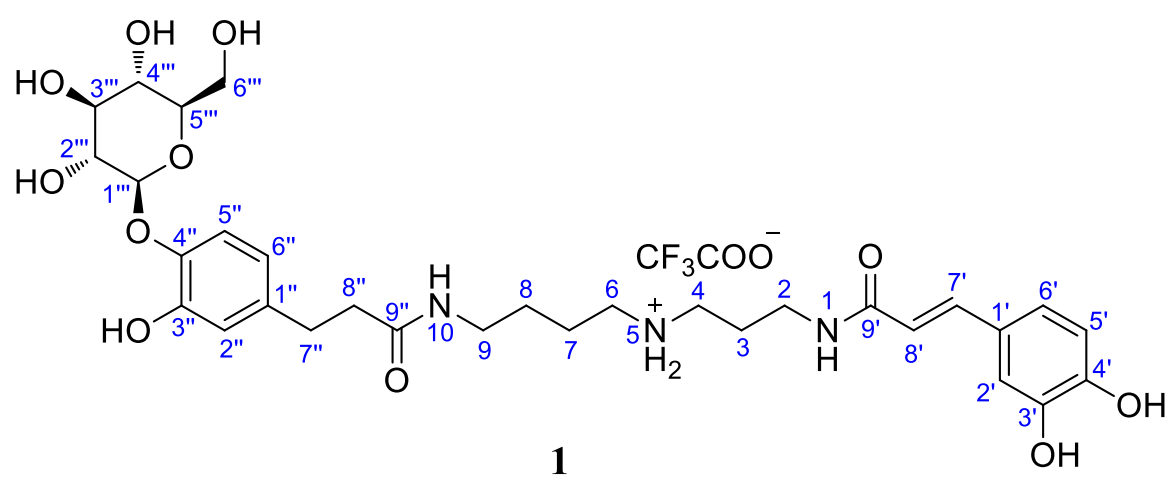

Table S1. NMR (600 MHz for ${ }^{1} \mathrm{H}$ NMR, $150 \mathrm{MHz}$ for ${ }^{13} \mathrm{C}$ NMR, DMSO- $d_{6}$ ) assignments of 1.

\begin{tabular}{|c|c|c|c|c|c|}
\hline No. & $\delta_{\mathrm{C}}$, type & $\delta_{\mathrm{H}}(J$ in $\mathrm{Hz})$ & ${ }^{1} \mathrm{H}-{ }^{1} \mathrm{H} \operatorname{COSY}$ & HMBC & NOESY \\
\hline 1 & & $8.21, \mathrm{t}(5.7)$ & 2 & $2,8^{\prime}, 9^{\prime}$ & $3,8^{\prime}$ \\
\hline 2 & $35.8, \mathrm{CH}_{2}$ & $3.23, q(6.4)$ & 1,3 & $3,4,9^{\prime}$ & \\
\hline 3 & $26.2, \mathrm{CH}_{2}$ & 1.78 , quint (6.7) & 2,4 & 2,4 & 1,5 \\
\hline 4 & $44.8, \mathrm{CH}_{2}$ & $2.90, \mathrm{~m}$ & 3,5 & $2,3,6$ & \\
\hline 5 & & 8.46 , br s & 4,6 & & 3,7 \\
\hline 6 & 46.6, $\mathrm{CH}_{2}$ & $2.88, \mathrm{~m}$ & 5,7 & $4,7,8$ & 8 \\
\hline 7 & $23.1, \mathrm{CH}_{2}$ & 1.53 , quint (7.1) & 6,8 & $6,8,9$ & 5 \\
\hline 8 & $26.3, \mathrm{CH}_{2}$ & 1.42 , quint (6.8) & 7,9 & $6,7,9$ & 6,10 \\
\hline 9 & $37.8, \mathrm{CH}_{2}$ & $3.04, q(6.5)$ & 8,10 & $7,8,9^{\prime \prime}$ & \\
\hline 10 & & $7.86, \mathrm{t}(5.6)$ & 9 & $8,9,9^{\prime \prime}$ & $8,8^{\prime \prime}$ \\
\hline $1^{\prime}$ & 126.3, C & & & & \\
\hline $2^{\prime}$ & $113.9, \mathrm{CH}$ & $6.95, \mathrm{~d}(1.9)$ & 6 & $3^{\prime}, 4^{\prime}, 5^{\prime}, 6^{\prime}, 7^{\prime}$ & $7^{\prime}, 8^{\prime}$ \\
\hline $3^{\prime}$ & 145.6, C & & & & \\
\hline $4^{\prime}$ & $147.5, \mathrm{C}$ & & & & \\
\hline $5^{\prime}$ & $115.8, \mathrm{CH}$ & $6.75, \mathrm{~d}(8.1)$ & $6^{\prime}$ & $1^{\prime}, 2^{\prime}, 3^{\prime}, 4^{\prime}$ & \\
\hline $6^{\prime}$ & $120.5, \mathrm{CH}$ & $6.84, \mathrm{dd}(8.2,1.9)$ & $2^{\prime}, 5^{\prime}$ & $2^{\prime}, 3^{\prime}, 4^{\prime}, 7^{\prime}$ & $7^{\prime}, 8^{\prime}$ \\
\hline $7^{\prime}$ & $139.5, \mathrm{CH}$ & $7.26, \mathrm{~d}(15.7)$ & $8^{\prime}$ & $1^{\prime}, 2^{\prime}, 6^{\prime}, 8^{\prime}, 9^{\prime}$ & $2^{\prime}, 6^{\prime}$ \\
\hline $8^{\prime}$ & $118.0, \mathrm{CH}$ & $6.32, \mathrm{~d}(15.7)$ & $7^{\prime}$ & $1^{\prime}, 7^{\prime}, 9^{\prime}$ & $1,2^{\prime}, 6^{\prime}$ \\
\hline $9^{\prime}$ & $166.0, \mathrm{C}$ & & & & \\
\hline $1 "$ & 136.3, C & & & & \\
\hline $2 "$ & $115.8, \mathrm{CH}$ & $6.65, \mathrm{~d}(2.0)$ & $6 "$ & 3", 4", 6", 7" & 7", 8" \\
\hline $3 "$ & 146.7, C & & & & \\
\hline $4 "$ & 143.6, C & & & & \\
\hline $5^{\prime \prime}$ & 117.0, $\mathrm{CH}$ & $6.99, \mathrm{~d}(8.2)$ & $6 "$ & 1", 2", 3", 4" & $1 " \prime$ \\
\hline $6 "$ & $118.9, \mathrm{CH}$ & $6.54, \mathrm{dd}(8.3,1.9)$ & $2 ", 5^{\prime \prime}$ & 2", 3", 4", 7" & 7" \\
\hline 7" & $30.6, \mathrm{CH}_{2}$ & $2.68, \mathrm{t}(7.4)$ & $8 "$ & 1", 2", 6", 8", 9" & $2 ", 6 "$ \\
\hline
\end{tabular}




\begin{tabular}{|c|c|c|c|c|c|}
\hline $8 "$ & $37.2, \mathrm{CH}_{2}$ & $2.30, \mathrm{t}(7.5)$ & $7 "$ & 1", 7", 9" & $10,2^{\prime \prime}$ \\
\hline $9 "$ & $171.4, \mathrm{C}$ & & & & \\
\hline $1 " '$ & $102.8, \mathrm{CH}$ & $4.59, \mathrm{~d}(7.3)$ & $2^{\prime \prime \prime}$ & 4", 3"', 4"', 5"' & $5 ", 3 " ', 5 "$ \\
\hline $2 " \prime$ & $73.4, \mathrm{CH}$ & $3.27, \mathrm{~m}$ & $1^{\prime \prime \prime}, 3^{\prime \prime \prime}$ & & \\
\hline $3 "$ & $75.9, \mathrm{CH}$ & $3.27, \mathrm{~m}$ & $2^{\prime \prime \prime}, 4 " '$ & & $1 " \prime$ \\
\hline $4 " '$ & $69.9, \mathrm{CH}$ & $3.16, \mathrm{~m}$ & $3^{\prime \prime \prime}, 5^{\prime \prime \prime}$ & & \\
\hline $5^{\prime \prime \prime}$ & $77.2, \mathrm{CH}$ & $3.28, \mathrm{~m}$ & 4"', 6"'-Ha, 6"'-Hb & & $1^{\prime \prime \prime}$ \\
\hline \multirow[t]{2}{*}{ 6"' } & $60.8, \mathrm{CH}_{2}$ & $3.71, \mathrm{dd}(11.8,1.7), \mathrm{Ha}$ & 5"', 6"'-Hb & $4 " '$ & \\
\hline & & 3.47 , dd $(11.9,5.9), \mathrm{Hb}$ & 5'", 6"'-Ha & $4 " ', 5$ "' & \\
\hline
\end{tabular}




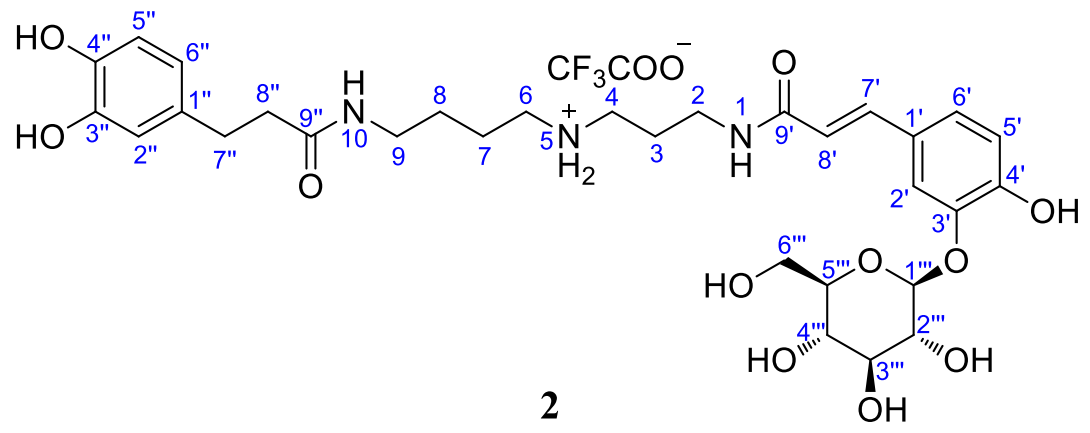

Table S2. NMR (600 MHz for ${ }^{1} \mathrm{H}$ NMR, $150 \mathrm{MHz}$ for ${ }^{13} \mathrm{C}$ NMR, DMSO- $\left.d_{6}\right)$ assignments of 2.

\begin{tabular}{|c|c|c|c|c|c|c|c|}
\hline No. & $\delta_{\mathrm{C}}$, type & $\delta_{\mathrm{H}}(J$ in $\mathrm{Hz})$ & ${ }^{1} \mathrm{H}-{ }^{1} \mathrm{H}$ COSY & HMBC & NOESY & $\delta_{\mathrm{C}}$, type $^{a}$ & $\delta_{\mathrm{H}}(J \text { in } \mathrm{Hz})^{a}$ \\
\hline 1 & & $8.14, \mathrm{t}(5.9)$ & 2 & $2,9^{\prime}$ & $8^{\prime}$ & & \\
\hline 2 & $35.8, \mathrm{CH}_{2}$ & $3.24, \mathrm{q}(6.5)$ & 1,3 & $3,4,9^{\prime}$ & & 37.0, $\mathrm{CH}_{2}$ & $3.39, \mathrm{~m}$ \\
\hline 3 & $26.2, \mathrm{CH}_{2}$ & 1.78 , quint (6.8) & 2,4 & 2,4 & & 27.7, $\mathrm{CH}_{2}$ & 1.91, quint (6.6) \\
\hline 4 & 44.7, $\mathrm{CH}_{2}$ & $2.89, \mathrm{~m}$ & 3,5 & & & $46.2, \mathrm{CH}_{2}$ & $2.98, \mathrm{t}(6.9)$ \\
\hline 5 & & 8.47 , br s & 4,6 & & & & \\
\hline 6 & $46.5, \mathrm{CH}_{2}$ & $2.88, \mathrm{~m}$ & 5,7 & & & $48.5, \mathrm{CH}_{2}$ & $2.92, \mathrm{t}(7.3)$ \\
\hline 7 & $23.1, \mathrm{CH}_{2}$ & 1.53 , quint (7.1) & 6,8 & 6 & & $24.4, \mathrm{CH}_{2}$ & $1.53, \mathrm{~m}$ \\
\hline 8 & $26.2, \mathrm{CH}_{2}$ & 1.41 , quint (7.3) & 7,9 & $6,7,9$ & & 27.3, $\mathrm{CH}_{2}$ & $1.48, \mathrm{~m}$ \\
\hline 9 & $37.7, \mathrm{CH}_{2}$ & $3.04, \mathrm{q}(6.1)$ & 8,10 & $7,8,9^{\prime \prime}$ & & $39.2, \mathrm{CH}_{2}$ & $3.14, \mathrm{t}(6.1)$ \\
\hline 10 & & $7.83, \mathrm{t}(5.6)$ & 9 & $9,9^{\prime \prime}$ & & & \\
\hline $1^{\prime}$ & $126.4, \mathrm{C}$ & & & & & 128.2, C & \\
\hline $2^{\prime}$ & $115.8, \mathrm{CH}$ & $7.35, \mathrm{~d}(1.7)$ & $6^{\prime}$ & $3^{\prime}, 4^{\prime}, 6^{\prime}, 7^{\prime}$ & $8^{\prime}, 1 " '$ & $117.2, \mathrm{CH}$ & $7.45, \mathrm{~d}(1.5)$ \\
\hline $3^{\prime}$ & $145.5, \mathrm{C}$ & & & & & 147.0, C & \\
\hline $4^{\prime}$ & $148.5, \mathrm{C}$ & & & & & $150.0, \mathrm{C}$ & \\
\hline $5^{\prime}$ & $116.2, \mathrm{CH}$ & $6.84, \mathrm{~d}(8.2)$ & $6^{\prime}$ & $1^{\prime}, 3^{\prime}, 4^{\prime}$ & & $117.3, \mathrm{CH}$ & $6.84, \mathrm{~d}(8.3)$ \\
\hline $6^{\prime}$ & $123.0, \mathrm{CH}$ & $7.10, \mathrm{dd}(8.2,1.6)$ & $2^{\prime}, 5^{\prime}$ & $2^{\prime}, 4^{\prime}, 7^{\prime}$ & $7^{\prime}, 8^{\prime}$ & $125.7, \mathrm{CH}$ & $7.11, \mathrm{dd}(8.3,1.5)$ \\
\hline $7^{\prime}$ & $139.2, \mathrm{CH}$ & 7.32, d (15.7) & $8^{\prime}$ & $1^{\prime}, 2^{\prime}, 6^{\prime}, 8^{\prime}, 9^{\prime}$ & $6^{\prime}$ & $142.4, \mathrm{CH}$ & 7.46, d (15.6) \\
\hline $8^{\prime}$ & $118.8, \mathrm{CH}$ & $6.41, \mathrm{~d}(15.8)$ & $7^{\prime}$ & $1^{\prime}, 9^{\prime}$ & $1,2^{\prime}, 6^{\prime}$ & $118.4, \mathrm{CH}$ & $6.42, \mathrm{~d}(15.7)$ \\
\hline $9^{\prime}$ & 165.9, C & & & & & 170.0, C & \\
\hline $1 "$ & 132.1, C & & & & & 133.6, C & \\
\hline $2 "$ & 115.6, $\mathrm{CH}$ & $6.56, \mathrm{~d}(1.9)$ & $6 "$ & 3", 4", 6", 7" & & $116.8, \mathrm{CH}$ & $6.62, \mathrm{~d}(1.8)$ \\
\hline $3 "$ & 145.0, C & & & & & 145.9, C & \\
\hline $4 "$ & 143.3, C & & & & & $144.4, \mathrm{C}$ & \\
\hline $5^{\prime \prime}$ & $115.4, \mathrm{CH}$ & $6.61, \mathrm{~d}(8.0)$ & $6 "$ & 1", 3", 4" & & $116.4, \mathrm{CH}$ & $6.66, \mathrm{~d}(8.0)$ \\
\hline 6" & $118.7, \mathrm{CH}$ & $6.41, \mathrm{~m}$ & $2 ", 5 "$ & 2", 4", 7" & & $120.8, \mathrm{CH}$ & $6.50, \mathrm{dd}(8.0,1.8)$ \\
\hline 7" & $30.6, \mathrm{CH}_{2}$ & $2.61, \mathrm{t}(7.5)$ & $8 "$ & 1", 2", 6", 8", 9" & & $32.3, \mathrm{CH}_{2}$ & $2.73, \mathrm{t}(7.4)$ \\
\hline $8 "$ & 37.6, $\mathrm{CH}_{2}$ & $2.26, \mathrm{t}(7.4)$ & 7" & 1", 7", 9" & & $39.2, \mathrm{CH}_{2}$ & $2.39, \mathrm{t}(7.2)$ \\
\hline $9^{\prime \prime}$ & $171.5, \mathrm{C}$ & & & & & 175.6, C & \\
\hline
\end{tabular}




\begin{tabular}{|c|c|c|c|c|c|c|c|}
\hline $1 " '$ & $102.1, \mathrm{CH}$ & $4.74, \mathrm{~d}(7.3)$ & $2^{\prime \prime \prime}$ & $3^{\prime}$ & 2', 3"', 5"' & $104.0, \mathrm{CH}$ & $4.80, \mathrm{~d}(7.1)$ \\
\hline 2"' & 73.3, $\mathrm{CH}$ & $3.31, \mathrm{~m}$ & $1 " ', 3^{\prime \prime \prime}$ & 1'", 3 "' & & $74.8, \mathrm{CH}$ & $3.51, \mathrm{~m}$ \\
\hline 3"' & $76.0, \mathrm{CH}$ & $3.29, \mathrm{~m}$ & 2"', 4"' & $2 " '$ & $1 " '$ & $77.5, \mathrm{CH}$ & $3.49, \mathrm{~m}$ \\
\hline 4"' & $69.9, \mathrm{CH}$ & $3.18, \mathrm{~m}$ & 3"', $5^{\prime \prime \prime}$ & $3^{\prime \prime \prime}$ & & $71.4, \mathrm{CH}$ & $3.39, \mathrm{~m}$ \\
\hline 5"' & $77.3, \mathrm{CH}$ & $3.36, \mathrm{~m}$ & 4"', 6"'-Ha, 6"'-Hb & 6"' & $1 " '$ & $78.4, \mathrm{CH}$ & $3.49, \mathrm{~m}$ \\
\hline \multirow[t]{2}{*}{ 6"' } & $60.7, \mathrm{CH}_{2}$ & 3.76 , dd $(11.8,1.6), \mathrm{Ha}$ & 5"', 6"'-Hb & & & $62.5, \mathrm{CH}_{2}$ & 3.94, br d (11.0), Ha \\
\hline & & $3.49, \mathrm{dd}(11.9,6.1), \mathrm{Hb}$ & 5"', 6"'-Ha & $5^{\prime \prime \prime}$ & & & $3.71, \mathrm{dd}(12.1,5.9), \mathrm{Hb}$ \\
\hline
\end{tabular}

${ }^{a}$ The data were Measured in $\mathrm{CD}_{3} \mathrm{OD}\left({ }^{1} \mathrm{H}\right.$ NMR for $300 \mathrm{MHz},{ }^{13} \mathrm{C}$ NMR for $\left.75 \mathrm{MHz}\right)$. 


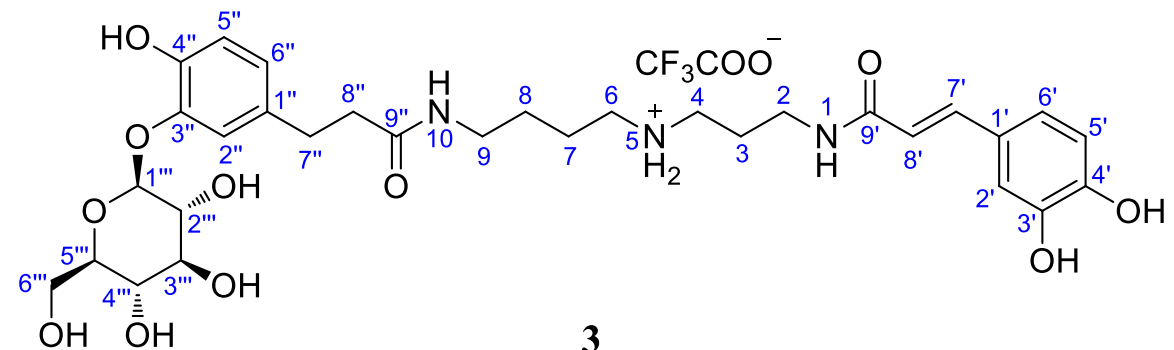

Table S3. NMR (600 MHz for ${ }^{1} \mathrm{H}$ NMR, $150 \mathrm{MHz}$ for ${ }^{13} \mathrm{C}$ NMR, DMSO- $\left.d_{6}\right)$ assignments of 3.

\begin{tabular}{|c|c|c|c|c|c|}
\hline No. & $\delta_{\mathrm{C}}$, type & $\delta_{\mathrm{H}}(J$ in $\mathrm{Hz})$ & ${ }^{1} \mathrm{H}-{ }^{1} \mathrm{H}$ COSY & HMBC & NOESY \\
\hline 1 & & $8.17, \mathrm{t}(5.3)$ & 2 & $2,9^{\prime}$ & $8^{\prime}$ \\
\hline 2 & $35.7, \mathrm{CH}_{2}$ & $3.23, q(6.4)$ & 1,3 & $3,4,9^{\prime}$ & \\
\hline 3 & $26.2, \mathrm{CH}_{2}$ & 1.77 , quint (7.1) & 2,4 & 2,4 & \\
\hline 4 & $44.7, \mathrm{CH}_{2}$ & $2.89, \mathrm{~m}$ & 3 & $2,3,6$ & \\
\hline 5 & & 8.34 , br s & & & \\
\hline 6 & $46.5, \mathrm{CH}_{2}$ & $2.89, \mathrm{~m}$ & 7 & $4,7,8$ & \\
\hline 7 & $23.1, \mathrm{CH}_{2}$ & 1.51 , quint (6.8) & 6,8 & $6,8,9$ & \\
\hline 8 & $26.2, \mathrm{CH}_{2}$ & 1.41, quint (7.4) & 7,9 & $6,7,9$ & \\
\hline 9 & 37.7, $\mathrm{CH}_{2}$ & $3.04, q(6.1)$ & 8,10 & $7,8,9^{\prime \prime}$ & \\
\hline 10 & & $7.81, \mathrm{t}(5.5)$ & 9 & $9,9^{\prime \prime}$ & $8 "$ \\
\hline $1^{\prime}$ & 126.2, C & & & & \\
\hline $2^{\prime}$ & $113.8, \mathrm{CH}$ & $6.94, \mathrm{~m}$ & $6^{\prime}$ & $3^{\prime}, 4^{\prime}, 5^{\prime}, 6^{\prime}, 7^{\prime}$ & $8^{\prime}$ \\
\hline $3^{\prime}$ & $145.5, \mathrm{C}$ & & & & \\
\hline $4^{\prime}$ & $147.4, \mathrm{C}$ & & & & \\
\hline $5^{\prime}$ & $115.7, \mathrm{CH}$ & $6.75, \mathrm{~d}(8.1)$ & $6^{\prime}$ & $1^{\prime}, 2^{\prime}, 3^{\prime}, 4^{\prime}, 6^{\prime}$ & \\
\hline $6^{\prime}$ & $120.5, \mathrm{CH}$ & $6.84, \mathrm{dd}(8.2,1.9)$ & $2^{\prime}, 5^{\prime}$ & $2^{\prime}, 3^{\prime}, 4^{\prime}, 5^{\prime}, 7^{\prime}$ & $8^{\prime}$ \\
\hline $7^{\prime}$ & $139.5, \mathrm{CH}$ & $7.26, \mathrm{~d}(15.6)$ & $8^{\prime}$ & $1^{\prime}, 2^{\prime}, 6^{\prime}, 8^{\prime}, 9^{\prime}$ & \\
\hline $8^{\prime}$ & $118.0, \mathrm{CH}$ & $6.31, \mathrm{~d}(15.7)$ & $7^{\prime}$ & $1^{\prime}, 7^{\prime}, 9^{\prime}$ & $1,2^{\prime}, 6^{\prime}$ \\
\hline $9^{\prime}$ & 166.0, C & & & & \\
\hline $1^{\prime \prime}$ & $132.2, \mathrm{C}$ & & & & \\
\hline $2^{\prime \prime}$ & $116.8, \mathrm{CH}$ & $6.95, \mathrm{~m}$ & 6", 7" & 1", 3", 4", 6", 7" & 1"', 7", 8" \\
\hline $3^{\prime \prime}$ & 145.0, C & & & & \\
\hline $4 "$ & $144.9, \mathrm{C}$ & & & & \\
\hline $5^{\prime \prime}$ & $115.6, \mathrm{CH}$ & $6.70, d(8.1)$ & $6 "$ & 1", 2", 3", 4" & \\
\hline $6^{\prime \prime}$ & $122.4, \mathrm{CH}$ & $6.68, \operatorname{dd}(8.2,1.6)$ & $2 ", 5 "$ & 1", 2", 3", 4", 7" & \\
\hline $7 "$ & $30.5, \mathrm{CH}_{2}$ & $2.68, \operatorname{td}(7.5,2.9)$ & $2 ", 8 "$ & 1", 2", 6", 8", 9" & $2 "$ \\
\hline $8^{\prime \prime}$ & $37.1, \mathrm{CH}_{2}$ & $2.31, \mathrm{t}(7.8)$ & 7" & 1", 7", 9" & $10,2^{\prime \prime}$ \\
\hline $9^{\prime \prime}$ & $171.4, \mathrm{C}$ & & & & \\
\hline $1 " '$ & $102.4, \mathrm{CH}$ & $4.62, \mathrm{~d}(7.3)$ & $2^{\prime \prime \prime}$ & 3", 3"', 5"' & 2", $3 " ', 5^{\prime \prime \prime}$ \\
\hline $2^{\prime \prime \prime}$ & $73.3, \mathrm{CH}$ & $3.28, \mathrm{~m}$ & 1"', 3 "', 2 "'-OH & & \\
\hline 3"' & $75.9, \mathrm{CH}$ & $3.27, \mathrm{~m}$ & 2"', 4"', 3 "'-OH & & $1 " '$ \\
\hline $4^{\prime \prime \prime}$ & $69.8, \mathrm{CH}$ & $3.16, \mathrm{~m}$ & 3"', 5"', 4"'-OH & & \\
\hline
\end{tabular}




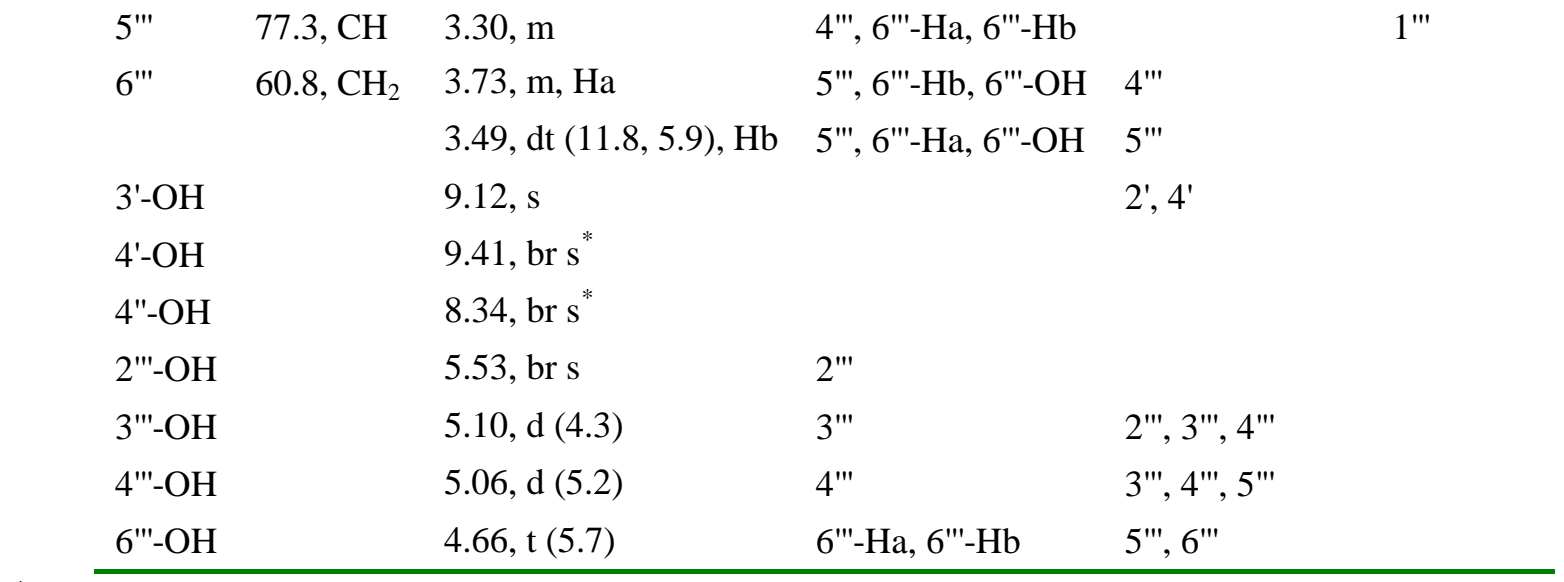

*Assignment may be interchanged. 


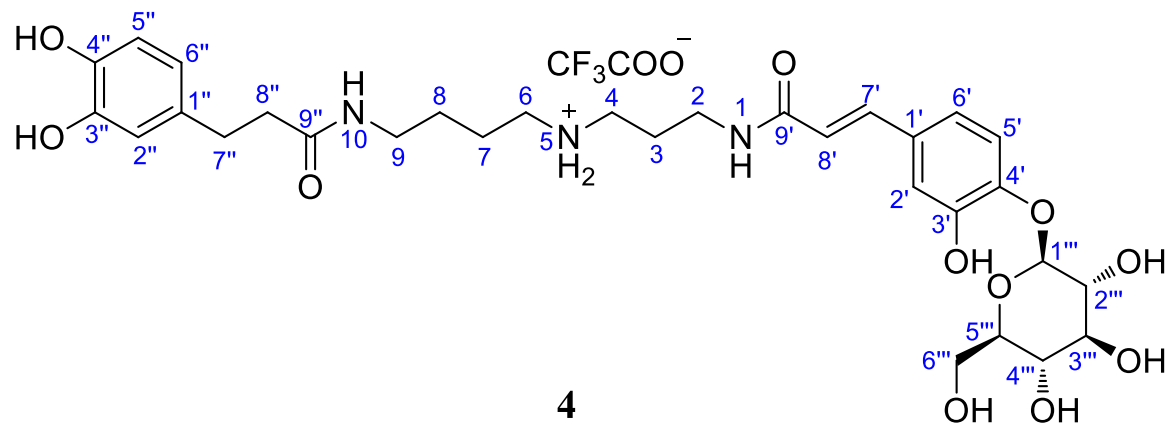

Table S4. NMR (600 MHz for ${ }^{1} \mathrm{H}$ NMR, $150 \mathrm{MHz}$ for ${ }^{13} \mathrm{C}$ NMR, DMSO- $d_{6}$ ) assignments of 4.

\begin{tabular}{|c|c|c|c|c|c|}
\hline No. & $\delta_{\mathrm{C}}$, type & $\delta_{\mathrm{H}}(J$ in $\mathrm{Hz})$ & ${ }^{1} \mathrm{H}-{ }^{1} \mathrm{H}$ COSY & HMBC & NOESY \\
\hline 1 & & 8.22 , br s & 2 & $2,9^{\prime}$ & $3,8^{\prime}$ \\
\hline 2 & $35.8, \mathrm{CH}_{2}$ & $3.24, q(5.7)$ & 1,3 & $3,4,9^{\prime}$ & \\
\hline 3 & $26.2, \mathrm{CH}_{2}$ & 1.77 , quint (6.8) & 2,4 & 2,4 & 1 \\
\hline 4 & 44.7, $\mathrm{CH}_{2}$ & $2.90, \mathrm{~m}$ & 3 & $2,3,6$ & \\
\hline 5 & & 8.34, br s & & & \\
\hline 6 & $46.5, \mathrm{CH}_{2}$ & $2.89, \mathrm{~m}$ & 7 & $4,7,8$ & \\
\hline 7 & 23.1, $\mathrm{CH}_{2}$ & 1.53 , quint (6.7) & 6,8 & $6,8,9$ & \\
\hline 8 & $26.2, \mathrm{CH}_{2}$ & 1.42 , quint (6.7) & 7,9 & $6,7,9$ & \\
\hline 9 & $37.7, \mathrm{CH}_{2}$ & $3.04, q(6.5)$ & 8,10 & $7,8,9^{\prime \prime}$ & \\
\hline 10 & & $7.82, \mathrm{t}(5.5)$ & 9 & $9,9^{\prime \prime}$ & \\
\hline $1^{\prime}$ & $129.4, \mathrm{C}$ & & & & \\
\hline $2^{\prime}$ & $114.1, \mathrm{CH}$ & 7.02, d (1.6) & $6^{\prime}$ & $3^{\prime}, 4^{\prime}, 6^{\prime}, 7^{\prime}$ & $7^{\prime}, 8^{\prime}$ \\
\hline $3^{\prime}$ & 146.9, C & & & & \\
\hline $4^{\prime}$ & 146.7, C & & & & \\
\hline $5^{\prime}$ & 116.3, $\mathrm{CH}$ & $7.11, \mathrm{~d}(8.4)$ & $6^{\prime}$ & $1^{\prime}, 2^{\prime}, 3^{\prime}, 4^{\prime}$ & $1 " '$ \\
\hline $6^{\prime}$ & $119.9, \mathrm{CH}$ & $6.96, \mathrm{dd}(8.5,1.8)$ & $2^{\prime}, 5^{\prime}$ & $2^{\prime}, 3^{\prime}, 4^{\prime}, 5^{\prime}, 7^{\prime}$ & $7^{\prime}, 8^{\prime}$ \\
\hline $7^{\prime}$ & $138.9, \mathrm{CH}$ & 7.31, d (15.7) & $8^{\prime}$ & $1^{\prime}, 2^{\prime}, 6^{\prime}, 9^{\prime}$ & $2^{\prime}, 6^{\prime}$ \\
\hline $8^{\prime}$ & $119.9, \mathrm{CH}$ & $6.43, \mathrm{~d}(15.5)$ & $7^{\prime}$ & $1^{\prime}, 7^{\prime}, 9^{\prime}$ & $1,2^{\prime}, 6^{\prime}$ \\
\hline $9^{\prime}$ & 165.7, C & & & & \\
\hline $1 "$ & 132.1, C & & & & \\
\hline $2^{\prime \prime}$ & $115.6, \mathrm{CH}$ & $6.55, \mathrm{~d}(1.9)$ & $6 "$ & 3", 4", 5", 6", 7" & $7 "$ \\
\hline $3 "$ & $144.9, \mathrm{C}$ & & & & \\
\hline $4 "$ & 143.3, C & & & & \\
\hline $5^{\prime \prime}$ & $115.4, \mathrm{CH}$ & $6.60, \mathrm{~d}(7.9)$ & $6 "$ & 1", 2", 3", 4" & \\
\hline 6" & $118.7, \mathrm{CH}$ & $6.41, \mathrm{dd}(7.9,2.0)$ & $2 ", 5 "$ & 2", 3", 4" ,5", 7" & $7 "$ \\
\hline 7" & $30.6, \mathrm{CH}_{2}$ & $2.62, \mathrm{t}(7.4)$ & $8 "$ & 1", 2", 6", 8", 9" & 2", 6" \\
\hline $8^{\prime \prime}$ & 37.6, $\mathrm{CH}_{2}$ & $2.26, \mathrm{t}(7.5)$ & $7^{\prime \prime}$ & 1", 7", 9" & \\
\hline 9" & $171.5, \mathrm{C}$ & & & & \\
\hline $1 " '$ & 101.7, CH & $4.75, \mathrm{~d}(7.3)$ & $2^{\prime \prime \prime}$ & 4', 3"', 5'"' & $5^{\prime}, 3^{\prime \prime \prime}, 5^{\prime \prime \prime}$ \\
\hline $2^{\prime \prime \prime}$ & 73.2, $\mathrm{CH}$ & $3.30, \mathrm{~m}$ & 1'", 3 "' & & \\
\hline
\end{tabular}




\begin{tabular}{|c|c|c|c|c|c|}
\hline 3"' & $75.8, \mathrm{CH}$ & $3.28, \mathrm{~m}$ & 2'", 4"', 3"'-OH & & $1 " '$ \\
\hline 4"' & $69.8, \mathrm{CH}$ & $3.17, \mathrm{~m}$ & 3'", 5"', 4"'-OH & & \\
\hline $5^{\prime \prime}$ & $77.2, \mathrm{CH}$ & $3.34, \mathrm{~m}$ & 4"', 6"'-Ha, 6"'-Hb & & $1 " '$ \\
\hline 6"' & $60.7, \mathrm{CH}_{2}$ & 3.72 , ddd $(11.6,5.3,1.9), \mathrm{Ha}$ & 5'", 6"'-Hb, 6"'-OH & 4"', 5"' & \\
\hline & & $3.47, \mathrm{dt}(11.8,6.0), \mathrm{Hb}$ & 5'", 6'"-Ha, 6"'-OH & 4"', 5"' & \\
\hline 3'-OH & & 8.79, br s$^{*}$ & & & \\
\hline 3"-OH & & 8.63 , br s & & 2", 3" & \\
\hline 4"-OH & & 8.69, br s$^{*}$ & & & \\
\hline 2"'-OH & & 5.42 , br s & & & \\
\hline 3"'-OH & & $5.10, \mathrm{~d}(4.3)$ & $3^{\prime \prime \prime}$ & 2"', 3"', 4"' & \\
\hline 4"'-OH & & $5.06, \mathrm{~d}(5.2)$ & $4 " '$ & 3"', 4'", 5"' & \\
\hline 6"'-OH & & $4.59, \mathrm{t}(5.6)$ & 6"'-Ha, 6"'-Hb & 5"', 6"' & \\
\hline
\end{tabular}

*Assignment may be interchanged. 


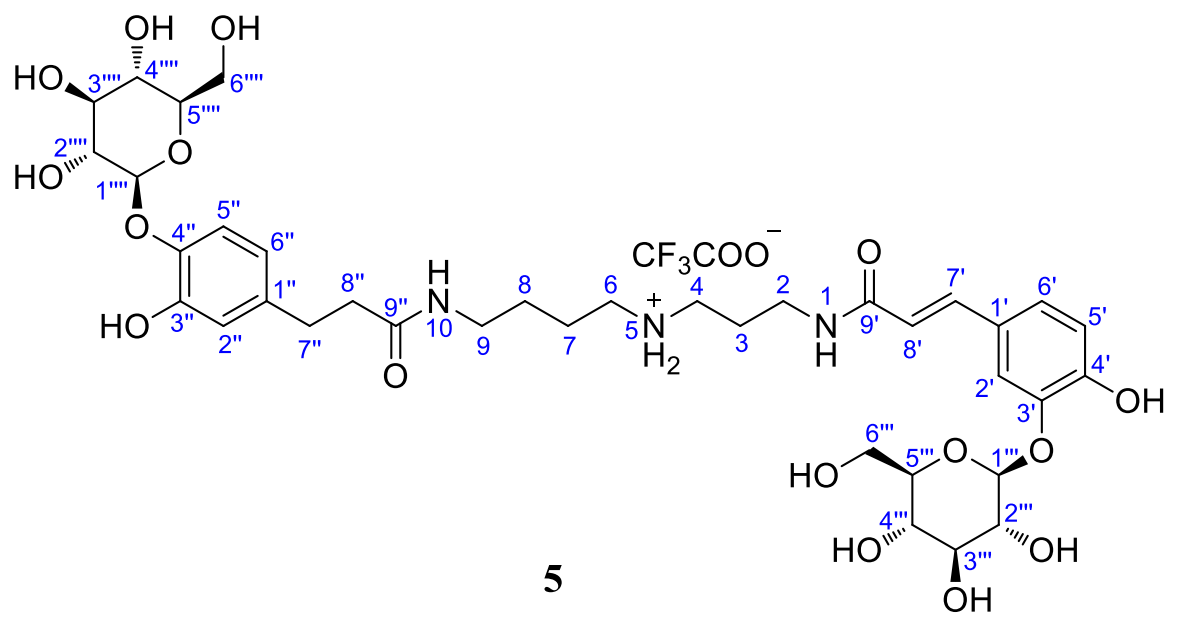

Table S5. NMR (600 MHz for ${ }^{1} \mathrm{H}$ NMR, $150 \mathrm{MHz}$ for ${ }^{13} \mathrm{C}$ NMR, DMSO- $d_{6}$ ) assignments of 5.

\begin{tabular}{|c|c|c|c|c|c|}
\hline No. & $\delta_{\mathrm{C}}$, type & $\delta_{\mathrm{H}}(J$ in $\mathrm{Hz})$ & ${ }^{1} \mathrm{H}-{ }^{1} \mathrm{H} \mathrm{COSY}$ & HMBC & NOESY \\
\hline 1 & & $8.10, \mathrm{t}(5.8)$ & 2 & $2,8^{\prime}, 9^{\prime}$ & $8^{\prime}, 3$ \\
\hline 2 & $35.8, \mathrm{CH}_{2}$ & $3.24, \mathrm{~m}$ & 1,3 & $3,4,9^{\prime}$ & \\
\hline 3 & $26.2, \mathrm{CH}_{2}$ & 1.77 , quint (6.8) & 2,4 & 2,4 & 1 \\
\hline 4 & $44.7, \mathrm{CH}_{2}$ & $2.89, \mathrm{~m}$ & 3,5 & $2,3,6$ & \\
\hline 5 & & 8.35 , br s & 4,6 & & \\
\hline 6 & $46.5, \mathrm{CH}_{2}$ & $2.89, \mathrm{~m}$ & 5,7 & $4,7,8$ & \\
\hline 7 & $23.1, \mathrm{CH}_{2}$ & 1.53 , quint (7.1) & 6,8 & $6,8,9$ & \\
\hline 8 & $26.2, \mathrm{CH}_{2}$ & 1.42 , quint (7.0) & 7,9 & $6,7,9$ & 10 \\
\hline 9 & $37.7, \mathrm{CH}_{2}$ & $3.04, \mathrm{q}(6.1)$ & 8,10 & $7,8,9^{\prime \prime}$ & \\
\hline 10 & & $7.84, \mathrm{t}(5.7)$ & 9 & $8,9,9^{\prime \prime}$ & $8,8^{\prime \prime}$ \\
\hline $1^{\prime}$ & $126.4, \mathrm{C}$ & & & & \\
\hline $2^{\prime}$ & $115.8, \mathrm{CH}$ & $7.35, \mathrm{~d}(1.9)$ & $6^{\prime}$ & $1^{\prime}, 3^{\prime}, 4^{\prime}, 6^{\prime}, 7^{\prime}$ & 8', 1"' \\
\hline $3^{\prime}$ & $145.5, \mathrm{C}$ & & & & \\
\hline $4^{\prime}$ & 148.5, C & & & & \\
\hline $5^{\prime}$ & $116.2, \mathrm{CH}$ & $6.84, \mathrm{~d}(8.2)$ & $6^{\prime}$ & $1^{\prime}, 2^{\prime}, 3^{\prime}, 4^{\prime}$ & \\
\hline $6^{\prime}$ & $122.9, \mathrm{CH}$ & $7.11, \mathrm{dd}(8.4,1.9)$ & $2^{\prime}, 5^{\prime}$ & $2^{\prime}, 3^{\prime}, 4^{\prime}, 7^{\prime}$ & $8^{\prime}$ \\
\hline $7^{\prime}$ & $139.3, \mathrm{CH}$ & $7.32, \mathrm{~d}(15.7)$ & $8^{\prime}$ & $1^{\prime}, 2^{\prime}, 6^{\prime}, 8^{\prime}, 9^{\prime}$ & \\
\hline $8^{\prime}$ & $118.8, \mathrm{CH}$ & $6.39, \mathrm{~d}(15.7)$ & $7^{\prime}$ & $1^{\prime}, 7^{\prime}, 9^{\prime}$ & $1,2^{\prime}, 6^{\prime}$ \\
\hline $9^{\prime}$ & 165.9, C & & & & \\
\hline $1 "$ & 136.2, C & & & & \\
\hline $2 "$ & $115.8, \mathrm{CH}$ & $6.64, \mathrm{~d}(2.1)$ & $6 "$ & 3", 4", 6", 7" & 7" \\
\hline $3 "$ & 146.6, C & & & & \\
\hline $4 "$ & 143.6, C & & & & \\
\hline $5 "$ & $116.9, \mathrm{CH}$ & $6.99, \mathrm{~d}(8.3)$ & $6 "$ & 1", 2", 3", 4" & $1^{\prime \prime \prime \prime}$ \\
\hline 6" & $118.9, \mathrm{CH}$ & $6.54, \mathrm{dd}(8.2,2.0)$ & $2 ", 5 "$ & 2", 3", 4", 7" & 7" \\
\hline 7" & $30.6, \mathrm{CH}_{2}$ & $2.68, \mathrm{t}(7.4)$ & $8 "$ & 1", 2", 6", 8", 9" & 2", 6" \\
\hline $8 "$ & $37.2, \mathrm{CH}_{2}$ & $2.30, \mathrm{t}(7.4)$ & $7 "$ & 1", 7", 9" & 10 \\
\hline
\end{tabular}




\begin{tabular}{|c|c|c|c|c|c|}
\hline $9^{\prime \prime}$ & 171.3, C & & & & \\
\hline $1 " '$ & $102.1, \mathrm{CH}$ & $4.74, \mathrm{~d}(7.4)$ & $2 " '$ & 3', 3"', 5"' & 2', 3'", 5"' \\
\hline $2^{\prime \prime \prime}$ & $73.3, \mathrm{CH}$ & $3.28, \mathrm{~m}$ & $1 " ', 3^{\prime \prime \prime}$ & & \\
\hline $3^{\prime \prime \prime}$ & $76.0^{* 1}, \mathrm{CH}$ & $3.28, \mathrm{~m}$ & 2"', 4"', 3 "'-OH & & $1^{\prime \prime \prime}$ \\
\hline $4 " '$ & $69.8, \mathrm{CH}$ & $3.17, \mathrm{~m}$ & 3"', 5"', 4"'-OH & & \\
\hline $5 " '$ & $77.3^{* 2}, \mathrm{CH}$ & $3.36, \mathrm{~m}^{* 4}$ & 4"', 6"'-Ha, 6"'-Hb & & $1^{\prime \prime \prime}$ \\
\hline \multirow[t]{2}{*}{ 6"' } & $60.8^{* 3}, \mathrm{CH}_{2}$ & 3.76 , ddd $(11.8,5.1,2.0), \mathrm{Ha}^{* 5}$ & 5"', 6"'-Hb, 6"'-OH & 4"', 5"' & \\
\hline & & 3.49, dt $(11.9,6.2), \mathrm{Hb}^{* 6}$ & 5"', 6"'-Ha, 6"'-OH & 4"', 5"' & \\
\hline $1^{\prime \prime \prime \prime}$ & $102.7, \mathrm{CH}$ & $4.58, \mathrm{~d}(7.5)$ & $2^{\prime \prime \prime \prime}$ & 4", 3'"', 4"'", 5"'"' & $5^{\prime \prime}, 3^{\prime \prime \prime}, 5^{\prime \prime \prime \prime}$ \\
\hline $2^{\prime \prime \prime \prime}$ & $73.3, \mathrm{CH}$ & $3.28, \mathrm{~m}$ & $1^{\prime \prime \prime ', ~} 3^{\prime \prime " '}$ & & \\
\hline $3^{\prime \prime \prime \prime}$ & $75.8^{* 1}, \mathrm{CH}$ & $3.28, \mathrm{~m}$ & 2"'", 4'"', 3 "''-OH & & $1^{\prime \prime \prime \prime}$ \\
\hline $4 " '+$ & $69.8, \mathrm{CH}$ & $3.17, \mathrm{~m}$ & 3"'", 5'"', 4"'--OH & & \\
\hline $5^{\prime \prime \prime \prime}$ & $77.2^{* 2}, \mathrm{CH}$ & $3.28, \mathrm{~m}^{* 4}$ & 4"'', 6"''-Ha, 6"''-Hb & & $1^{\prime \prime \prime \prime}$ \\
\hline \multirow[t]{2}{*}{ 6"'" } & $60.7^{* 3}, \mathrm{CH}_{2}$ & $3.71, \operatorname{ddd}(11.8,5.2,1.9), \mathrm{Ha}^{* 5}$ & 5"', 6"''-Hb, 6"'"-OH & $4^{\prime \prime \prime}$ & \\
\hline & & $3.47, \mathrm{dt}(11.9,6.0), \mathrm{Hb}^{* 6}$ & 5"'", 6"''-Ha, 6"'"-OH & 4"'", 5"'"' & \\
\hline 4'-OH & & 9.06 , br s & & $5^{\prime}$ & \\
\hline 3"-OH & & 8.43, br s & & $2 "$ & \\
\hline 2"'-OH & & 5.53, br s $^{* 7}$ & & & \\
\hline 3"'-OH & & $5.11, \mathrm{~d}(4.6)^{* 8}$ & 3"' & 2"', 3 "', 4"' & \\
\hline 4"'-OH & & $5.08, \mathrm{~d}(5.3)^{* 9}$ & 4"' & 3"', 4"', 5"' & \\
\hline 6"'-OH & & $4.64, \mathrm{t}(5.9)^{* 10}$ & 6"'-Ha, 6"'-Hb & 5"', 6"' & \\
\hline 2"'--OH & & 5.49, br s $^{* 7}$ & & & \\
\hline 3"'--OH & & $5.08, \mathrm{~d}(5.3)^{* 8}$ & $3 " '+$ & 2"'", 3"', 4 '"' & \\
\hline 4"'--OH & & $5.04, \mathrm{~d}(5.3)^{* 9}$ & $4 " '$ & 3"'", 4 "'", $5^{\prime \prime \prime \prime}$ & \\
\hline 6"'-'-OH & & $4.59, \mathrm{t}(5.8)^{* 10}$ & 6"'--Ha, 6"'--Hb & $5^{\prime " ',}, 6 " '$ & \\
\hline
\end{tabular}

* Assignment may be interchanged. 


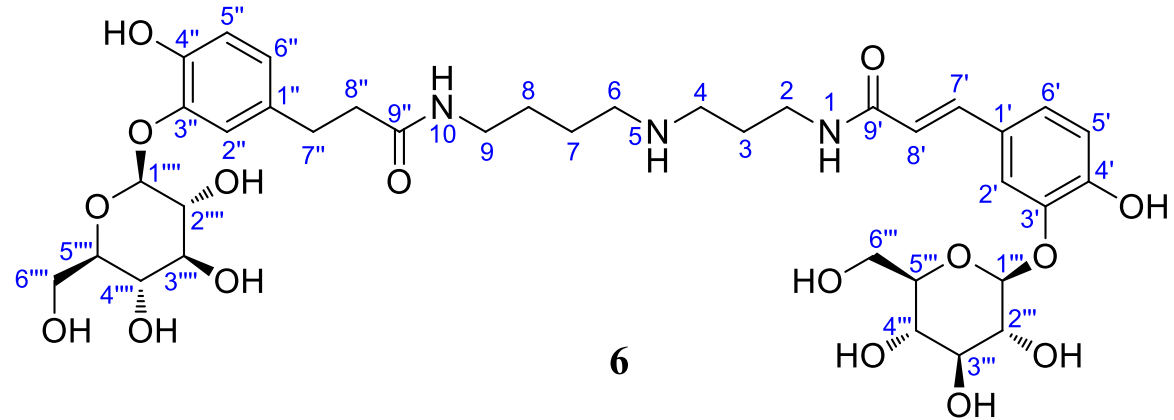

Table S6. NMR (600 MHz for ${ }^{1} \mathrm{H}$ NMR, $150 \mathrm{MHz}$ for ${ }^{13} \mathrm{C}$ NMR, DMSO- $d_{6}$ ) assignments of 6 .

\begin{tabular}{|c|c|c|c|c|c|}
\hline No. & $\delta_{\mathrm{C}}$, type & $\delta_{\mathrm{H}}(J$ in $\mathrm{Hz})$ & ${ }^{1} \mathrm{H}-{ }^{1} \mathrm{H}$ COSY & HMBC & NOESY \\
\hline 1 & & 7.88, br s & 2 & $2,9^{\prime}$ & $3,8^{\prime}$ \\
\hline 2 & $36.9, \mathrm{CH}_{2}$ & $3.18, \mathrm{~m}$ & 1,3 & $3,4,9^{\prime}$ & \\
\hline 3 & $29.5, \mathrm{CH}_{2}$ & 1.58, quint (6.6) & 2,4 & 2,4 & 1 \\
\hline 4 & $46.8, \mathrm{CH}_{2}$ & $2.51, \mathrm{t}(6.5)$ & 3 & $2,3,6$ & \\
\hline 5 & & & & & \\
\hline 6 & $48.9, \mathrm{CH}_{2}$ & $2.46, \mathrm{t}(6.6)$ & 7 & $4,7,8$ & \\
\hline 7 & $26.8, \mathrm{CH}_{2}$ & $1.35, \mathrm{~m}$ & 6,8 & $6,8,9$ & \\
\hline 8 & $27.1, \mathrm{CH}_{2}$ & $1.37, \mathrm{~m}$ & 7,9 & $6,7,9$ & 10 \\
\hline 9 & $38.4, \mathrm{CH}_{2}$ & $3.01, q(5.8)$ & 8,10 & $7,8,9^{\prime \prime}$ & \\
\hline 10 & & $7.74, \mathrm{t}(5.1)$ & 9 & $8,9,9^{\prime \prime}$ & $8,8^{\prime \prime}$ \\
\hline $1^{\prime}$ & 125.1, C & & & & \\
\hline $2^{\prime}$ & $116.2, \mathrm{CH}$ & $7.30, \mathrm{~d}(1.2)$ & $6^{\prime}$ & $3^{\prime}, 4^{\prime}, 6^{\prime}, 7^{\prime}$ & $1^{\prime \prime \prime}, 7 ', 8^{\prime}$ \\
\hline $3^{\prime}$ & 146.0, C & & & & \\
\hline $4^{\prime}$ & $150.8, \mathrm{C}$ & & & & \\
\hline $5^{\prime}$ & $116.8, \mathrm{CH}$ & $6.76, \mathrm{~d}(8.3)$ & $6^{\prime}$ & $1^{\prime}, 3^{\prime}, 4^{\prime}$ & \\
\hline $6^{\prime}$ & $123.3, \mathrm{CH}$ & $7.06, \mathrm{dd}(8.3,1.3)$ & $2^{\prime}, 5^{\prime}$ & $2^{\prime}, 4^{\prime}, 7^{\prime}$ & $7^{\prime}, 8^{\prime}$ \\
\hline $7^{\prime}$ & $139.0, \mathrm{CH}$ & $7.27, \mathrm{~d}(15.5)$ & $8^{\prime}$ & $1^{\prime}, 2^{\prime}, 6^{\prime}, 8^{\prime}, 9^{\prime}$ & $2^{\prime}, 6^{\prime}$ \\
\hline $8^{\prime}$ & $118.3, \mathrm{CH}$ & $6.34, \mathrm{~d}(15.7)$ & $7^{\prime}$ & $1^{\prime}, 9^{\prime}$ & $1,2^{\prime}, 6^{\prime}$ \\
\hline $9^{\prime}$ & $165.5, \mathrm{C}$ & & & & \\
\hline $1 "$ & $132.2, \mathrm{C}$ & & & & \\
\hline $2^{\prime \prime}$ & $116.9, \mathrm{CH}$ & 6.96 , br s & 6", 7" & 1", 3", 4", 6", 7" & $1 " ',, 7 ", 8 "$ \\
\hline $3 "$ & 145.1, C & & & & \\
\hline $4^{\prime \prime}$ & 145.1, C & & & & \\
\hline $5^{\prime \prime}$ & $115.7, \mathrm{CH}$ & $6.68, \mathrm{~s}$ & & 1", 3", 4" & \\
\hline $6^{\prime \prime}$ & $122.5, \mathrm{CH}$ & $6.68, \mathrm{~s}$ & $2^{\prime \prime}$ & 2", 4", 7" & $7 "$ \\
\hline $7^{\prime \prime}$ & $30.6, \mathrm{CH}_{2}$ & $2.67, \operatorname{td}(8.2,2.9)$ & 2", 8" & 1", 2", 6", 8", 9" & $2 ", 6 "$ \\
\hline $8^{\prime \prime}$ & $37.2, \mathrm{CH}_{2}$ & $2.30, \mathrm{t}(7.7)$ & $7^{\prime \prime}$ & 1", 7", 9" & $10,2^{\prime \prime}$ \\
\hline $9^{\prime \prime}$ & 171.3, C & & & & \\
\hline $1^{\prime \prime \prime}$ & $102.6, \mathrm{CH}$ & $4.69, \mathrm{~d}(7.1)$ & $2^{\prime \prime \prime}$ & 3', 3"' & $2^{\prime}, 3^{\prime \prime}, 5^{\prime \prime \prime}$ \\
\hline $2^{\prime \prime \prime}$ & $73.4, \mathrm{CH}$ & $3.29, \mathrm{~m}$ & 1'", $3^{\prime \prime \prime}$ & & \\
\hline $3^{\prime \prime \prime}$ & $76.2^{* 1}, \mathrm{CH}$ & $3.29, \mathrm{~m}$ & 2"', 4"' & & $1 " '$ \\
\hline
\end{tabular}




\begin{tabular}{|c|c|c|c|c|c|}
\hline 4 '" & $69.9, \mathrm{CH}$ & $3.18, \mathrm{~m}$ & $3^{\prime \prime}, 5^{\prime \prime \prime}$ & & \\
\hline $5^{\prime \prime \prime}$ & $77.3, \mathrm{CH}$ & $3.33, \mathrm{~m}^{* 3}$ & 4"', 6"'-Ha, 6"'-Hb & & $1 " '$ \\
\hline \multirow[t]{2}{*}{ 6"' } & $60.9^{* 2}, \mathrm{CH}_{2}$ & 3.76, br d (12.0), $\mathrm{Ha}^{* 4}$ & 5'", 6"'-Hb & $4 " '$ & \\
\hline & & 3.49 , dd $(11.8,5.9), \mathrm{Hb}$ & 5"', 6"'-Ha & 4"', 5"' & \\
\hline $1^{\prime \prime \prime \prime}$ & $102.5, \mathrm{CH}$ & $4.62, \mathrm{~d}(7.2)$ & $2^{\prime \prime \prime \prime}$ & 3", $3^{\prime \prime \prime}, 5^{\prime \prime \prime}$ & 2", $3^{\prime \prime \prime}, 5^{\prime \prime \prime}$ \\
\hline $2^{\prime \prime \prime \prime}$ & $73.4, \mathrm{CH}$ & $3.29, \mathrm{~m}$ & 1'"', $3^{\prime \prime \prime}$ & & \\
\hline $3^{\prime \prime \prime \prime}$ & $76.0^{* 1}, \mathrm{CH}$ & $3.29, \mathrm{~m}$ & $2^{\prime \prime \prime}, 4 " '+$ & & $1^{\prime \prime \prime \prime}$ \\
\hline $4^{\prime \prime \prime \prime}$ & $69.9, \mathrm{CH}$ & $3.18, \mathrm{~m}$ & $3^{\prime \prime \prime}, 5^{\prime \prime \prime}$ & & \\
\hline $5^{\prime \prime \prime \prime}$ & 77.3, $\mathrm{CH}$ & $3.29, \mathrm{~m}^{* 3}$ & 4'"', 6"'"-Ha, 6"''-Hb & & $1^{\prime \prime \prime \prime}$ \\
\hline \multirow[t]{2}{*}{$6^{\prime \prime \prime \prime}$} & $60.8^{* 2}, \mathrm{CH}_{2}$ & 3.74, br d (11.2), $\mathrm{Ha}^{* 4}$ & $5^{\prime \prime \prime}, 6$ "'"-Hb & 4"'", 5"'" & \\
\hline & & 3.49 , dd $(11.8,5.9), \mathrm{Hb}$ & 5'"', 6"'--Ha & 4"', 5 "'" & \\
\hline
\end{tabular}

*Assignment may be interchanged. 


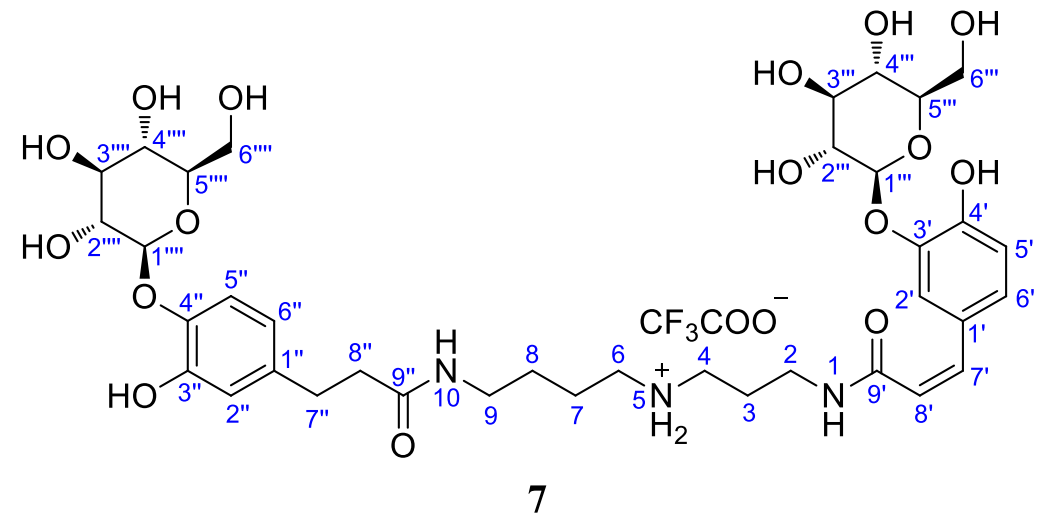

Table S7. NMR (300 MHz for ${ }^{1} \mathrm{H}$ NMR, $75 \mathrm{MHz}$ for ${ }^{13} \mathrm{C}$ NMR, DMSO- $d_{6}$ ) assignments of 7.

\begin{tabular}{|c|c|c|c|c|c|}
\hline No. & $\delta_{\mathrm{C}}$, type & $\delta_{\mathrm{H}}(J$ in $\mathrm{Hz})$ & ${ }^{1} \mathrm{H}-{ }^{1} \mathrm{H}$ COSY & $\mathrm{HMBC}^{a}$ & NOESY \\
\hline 1 & & $8.25, \mathrm{t}(5.5)$ & 2 & $2,3,8^{\prime}, 9^{\prime}$ & $8^{\prime}$ \\
\hline 2 & $35.8, \mathrm{CH}_{2}$ & $3.17, \mathrm{q}(4.8)$ & 1,3 & $3,4,9^{\prime}$ & \\
\hline 3 & 25.9, $\mathrm{CH}_{2}$ & 1.76 , quint (5.8) & 2,4 & 2,4 & \\
\hline 4 & $44.9, \mathrm{CH}_{2}$ & $2.86, \mathrm{~m}$ & 3,5 & $2,3,6$ & \\
\hline 5 & & 8.47 , br s & 4,6 & 4,6 & \\
\hline 6 & $46.6, \mathrm{CH}_{2}$ & $2.86, \mathrm{~m}$ & 5,7 & 4,7 & \\
\hline 7 & $23.1, \mathrm{CH}_{2}$ & 1.52 , quint (6.4) & 6,8 & $6,8,9$ & \\
\hline 8 & 26.3, $\mathrm{CH}_{2}$ & 1.40 , quint (6.9) & 7,9 & $6,7,9$ & \\
\hline 9 & $37.8, \mathrm{CH}_{2}$ & $3.03, \mathrm{q}(5.8)$ & 8,10 & $7,8,9^{\prime \prime}$ & \\
\hline 10 & & $7.89, \mathrm{t}(5.4)$ & 9 & $8,9,8^{\prime \prime}, 9^{\prime \prime}$ & $8 "$ \\
\hline $1^{\prime}$ & 126.9, C & & & & \\
\hline $2^{\prime}$ & 119.0, CH & $7.81, \mathrm{~d}(1.7)$ & $6^{\prime}$ & $3^{\prime}, 4^{\prime}, 5^{\prime}, 6^{\prime}, 7^{\prime}$ & 7', 1"' \\
\hline $3^{\prime}$ & 144.7, C & & & & \\
\hline $4^{\prime}$ & 147.7, C & & & & \\
\hline $5^{\prime}$ & $115.4, \mathrm{CH}$ & $6.77, \mathrm{~d}(8.3)$ & $6^{\prime}$ & $1^{\prime}, 2^{\prime}, 3^{\prime}, 4^{\prime}$ & \\
\hline $6^{\prime}$ & 126.1, CH & $7.24, \mathrm{dd}(8.4,1.6)$ & $2^{\prime}, 5^{\prime}$ & $2^{\prime}, 3^{\prime}, 4^{\prime}, 7^{\prime}$ & \\
\hline $7^{\prime}$ & $137.4, \mathrm{CH}$ & $6.52, \mathrm{~d}(13.2)$ & $8^{\prime}$ & $2^{\prime}, 6^{\prime}, 9^{\prime}$ & $2^{\prime}$ \\
\hline $8^{\prime}$ & $120.9, \mathrm{CH}$ & $5.80, \mathrm{~d}(12.9)$ & $7^{\prime}$ & $1^{\prime}, 7^{\prime}, 9^{\prime}$ & 1 \\
\hline $9^{\prime}$ & 166.6, C & & & & \\
\hline $1 "$ & 136.2, C & & & & \\
\hline $2 "$ & $115.9, \mathrm{CH}$ & $6.64, \mathrm{~d}(1.8)$ & $6 "$ & $3^{\prime}, 4^{\prime}, 6^{\prime}, 7^{\prime}$ & \\
\hline 3" & 146.7, C & & & & \\
\hline $4 "$ & 143.7, C & & & & \\
\hline $5^{\prime \prime}$ & $116.9, \mathrm{CH}$ & $6.99, \mathrm{~d}(8.2)$ & $6 "$ & $1^{\prime}, 2^{\prime}, 3^{\prime}, 4^{\prime}$ & $1^{\prime \prime \prime}$ \\
\hline $6 "$ & $119.0, \mathrm{CH}$ & $6.54, \mathrm{dd}(8.2,1.7)$ & $2^{\prime \prime}, 5^{\prime \prime}$ & $2^{\prime}, 3^{\prime}, 4^{\prime}, 7^{\prime}$ & \\
\hline 7" & $30.7, \mathrm{CH}_{2}$ & $2.67, \mathrm{t}(7.3)$ & $8^{\prime \prime}$ & $1^{\prime}, 2^{\prime}, 6^{\prime}, 8^{\prime}, 9^{\prime}$ & \\
\hline $8 "$ & $37.3, \mathrm{CH}_{2}$ & $2.29, \mathrm{t}(7.1)$ & $7 "$ & $1^{\prime}, 7^{\prime}, 9^{\prime}$ & 10 \\
\hline 9" & $171.5, \mathrm{C}$ & & & & \\
\hline $1 " \prime$ & $102.4, \mathrm{CH}$ & $4.65, \mathrm{~d}(7.1)$ & $2^{\prime \prime \prime}$ & 3', 3'", 5"' & $2^{\prime}, 3^{\prime \prime \prime}, 5^{\prime \prime \prime}$ \\
\hline
\end{tabular}




\begin{tabular}{|c|c|c|c|c|c|}
\hline $2^{\prime \prime \prime}$ & $73.3^{* 1}, \mathrm{CH}$ & $3.29, \mathrm{~m}$ & 1'", 3 "' & & \\
\hline $3^{\prime \prime \prime}$ & $75.9^{* 2}, \mathrm{CH}$ & $3.29, \mathrm{~m}$ & 2 '", 4"' & & $1 " '$ \\
\hline $4 " '$ & $69.6^{* 3}, \mathrm{CH}$ & $3.20, \mathrm{~m}$ & $3^{\prime \prime \prime}, 5^{\prime \prime \prime}$ & & \\
\hline $5^{\prime \prime \prime}$ & $77.2^{* 4}, \mathrm{CH}$ & $3.29, \mathrm{~m}$ & 4'", 6"'-Hb & & $1 " '$ \\
\hline \multirow[t]{2}{*}{ 6"' } & $60.8, \mathrm{CH}_{2}$ & $3.75, \mathrm{~d}(11.7), \mathrm{Ha}^{* 5}$ & 6"'-Hb & 4"', 5"' & \\
\hline & & $3.48, \mathrm{~m}, \mathrm{Hb}$ & 5'", 6"'-Ha & 4"', 5"' & \\
\hline $1 " '+$ & 102.7, CH & $4.59, \mathrm{~d}(7.1)$ & $2 " '$ & 4", 3"', 4"'", 5"' & 5", 3"'", 5"'" \\
\hline $2^{\prime \prime \prime}$ & $73.4^{* 1}, \mathrm{CH}$ & $3.29, \mathrm{~m}$ & 1'"', 3 "'" & & \\
\hline $3^{\prime \prime \prime \prime}$ & $76.0^{* 2}, \mathrm{CH}$ & $3.29, \mathrm{~m}$ & $2^{\prime \prime \prime \prime}, 4 " ' "$ & & $1^{\prime \prime \prime \prime}$ \\
\hline $4 " '+$ & $69.9^{* 3}, \mathrm{CH}$ & $3.20, \mathrm{~m}$ & $3^{\prime \prime \prime \prime}, 5^{\prime \prime \prime}$ & & \\
\hline $5^{\prime \prime \prime}$ & $77.3^{* 4}, \mathrm{CH}$ & $3.29, \mathrm{~m}$ & 4'"', 6"'--Hb & & $1^{\prime \prime \prime}$ \\
\hline \multirow[t]{2}{*}{$6^{\prime \prime \prime \prime}$} & $60.8, \mathrm{CH}_{2}$ & $3.71, \mathrm{~d}(11.9), \mathrm{Ha}^{* 5}$ & 6'"'-Hb & $4^{\prime \prime \prime \prime}$ & \\
\hline & & $3.48, \mathrm{~m}, \mathrm{Hb}$ & 5'"', 6"'--Ha & 4"'", 5"'" & \\
\hline 4'-OH & & 8.99, br s $^{* 6}$ & & & \\
\hline 3"-OH & & 8.47, br s $^{* 6}$ & & & \\
\hline
\end{tabular}

${ }^{a}$ The data were recorded with a Bruker AV 600 spectrometer. * Assignment may be interchanged. 


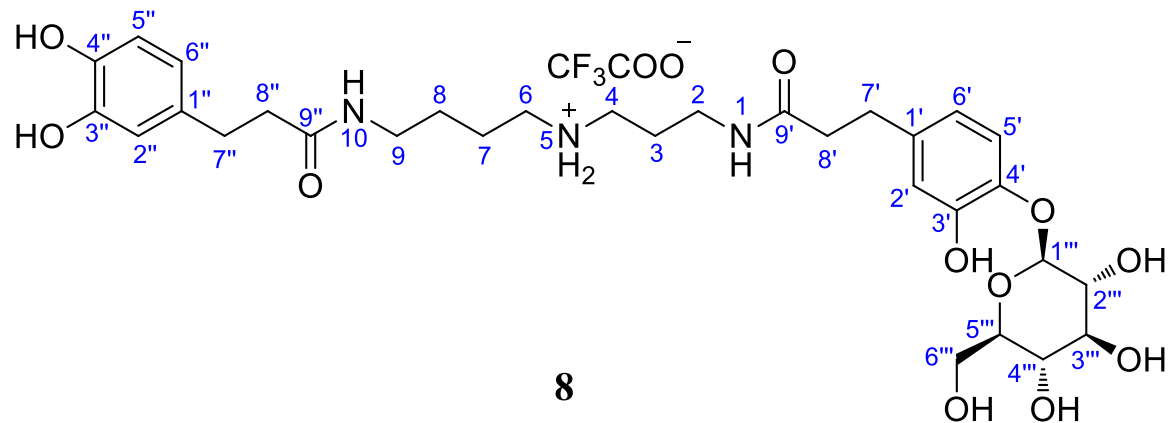

Table S8. NMR (600 MHz for ${ }^{1} \mathrm{H}$ NMR, $150 \mathrm{MHz}$ for ${ }^{13} \mathrm{C}$ NMR, DMSO- $\left.d_{6}\right)$ assignments of 8.

\begin{tabular}{|c|c|c|c|c|c|}
\hline No. & $\delta_{\mathrm{C}}$, type & $\delta_{\mathrm{H}}(J$ in $\mathrm{Hz})$ & ${ }^{1} \mathrm{H}-{ }^{1} \mathrm{H}$ COSY & HMBC & NOESY \\
\hline 1 & & $8.01 / 7.98, \mathrm{t}(5.8)$ & 2 & $2,9^{\prime}$ & $3,8^{\prime}$ \\
\hline 2 & $35.5, \mathrm{CH}_{2}$ & $3.10, \mathrm{q}(6.1)$ & 1,3 & $3,4,9^{\prime}$ & \\
\hline 3 & $26.1, \mathrm{CH}_{2}$ & 1.68 , quint (6.6) & 2,4 & 2,4 & 1,5 \\
\hline 4 & $44.6, \mathrm{CH}_{2}$ & $2.78, \mathrm{~m}$ & 3,5 & $2,3,6$ & \\
\hline 5 & & 8.37 , br s & 4,6 & & 3,7 \\
\hline 6 & $46.6, \mathrm{CH}_{2}$ & $2.84, \mathrm{~m}$ & 5,7 & $4,7,8$ & \\
\hline 7 & $23.1, \mathrm{CH}_{2}$ & 1.52 , quint (7.1) & 6,8 & $6,8,9$ & 5 \\
\hline 8 & 26.3, $\mathrm{CH}_{2}$ & 1.41 , quint (6.9) & 7,9 & $6,7,9$ & 10 \\
\hline 9 & $37.8 / 37.6, \mathrm{CH}_{2}$ & $3.04, \mathrm{q}(6.1)$ & 8,10 & $7,8,9^{\prime \prime}$ & \\
\hline 10 & & $7.86 / 7.84, \mathrm{t}(5.7)$ & 9 & $9,9 "$ & $8,8^{\prime \prime}$ \\
\hline $1^{\prime}$ & 136.2/136.1, C & & & & \\
\hline $2^{\prime}$ & $115.8, \mathrm{CH}$ & $6.65, \mathrm{~d}(2.2)$ & $6^{\prime}$ & $3^{\prime}, 4^{\prime}, 6^{\prime}, 7^{\prime}$ & $7^{\prime}, 8^{\prime}$ \\
\hline $3^{\prime}$ & $146.7, \mathrm{C}$ & & & & \\
\hline $4^{\prime}$ & 143.6, C & & & & \\
\hline $5^{\prime}$ & $116.9, \mathrm{CH}$ & $6.99, \mathrm{~d}(8.2)$ & $6^{\prime}$ & $1^{\prime}, 3^{\prime}, 4^{\prime}, 6^{\prime}$ & $1^{\prime \prime \prime}$ \\
\hline 6 & $118.9, \mathrm{CH}$ & $6.55 / 6.54, \mathrm{dd}(8.3,2.3)$ & $2^{\prime}, 5^{\prime}$ & $2^{\prime}, 4^{\prime}, 7^{\prime}$ & $7^{\prime}, 8^{\prime}$ \\
\hline $7^{\prime}$ & $30.6 / 30.5, \mathrm{CH}_{2}$ & $2.69, \mathrm{t}(7.4)$ & $8^{\prime}$ & $1^{\prime}, 2^{\prime}, 6^{\prime}, 8^{\prime}, 9^{\prime}$ & $2^{\prime}, 6^{\prime}$ \\
\hline $8^{\prime}$ & $37.0, \mathrm{CH}_{2}$ & $2.32, \mathrm{~m}$ & $7^{\prime}$ & $1^{\prime}, 7^{\prime}, 9^{\prime}$ & $1,2^{\prime}, 6^{\prime}$ \\
\hline $9^{\prime}$ & 172.2/172.1, C & & & & \\
\hline $1 "$ & 132.1/132.0, C & & & & \\
\hline $2^{\prime \prime}$ & $115.7, \mathrm{CH}$ & $6.56, \mathrm{~m}$ & $6 "$ & 3", 4", 6", 7" & $7^{\prime \prime}$ \\
\hline $3^{\prime \prime}$ & 145.0, C & & & & \\
\hline $4 "$ & 143.3, C & & & & \\
\hline $5^{\prime \prime}$ & $115.4, \mathrm{CH}$ & $6.61, \mathrm{~d}(8.0)$ & $6 "$ & 1", 2", 3", 4", 6" & \\
\hline $6^{\prime \prime}$ & $118.7, \mathrm{CH}$ & $6.42 / 6.41, \mathrm{dd}(8.0,2.3)$ & $2 ", 5 "$ & 2", 3", 4", 7" & $7^{\prime \prime}$ \\
\hline $7^{\prime \prime}$ & $30.6 / 30.5, \mathrm{CH}_{2}$ & $2.61, \mathrm{t}(7.9)$ & $8 "$ & 1", 2", 6", 8", 9" & $2 ", 6^{\prime \prime}$ \\
\hline $8^{\prime \prime}$ & $37.4 / 37.2, \mathrm{CH}_{2}$ & $2.28, \mathrm{~m}$ & 7" & 1", 7", 9" & 10 \\
\hline $9^{\prime \prime}$ & 171.6/171.4, C & & & & \\
\hline $1^{\prime \prime \prime}$ & 102.7, $\mathrm{CH}$ & $4.58, \mathrm{~d}(6.8)$ & $2^{\prime \prime \prime}$ & 4', 3"', 4"', 5"' & $5^{\prime}, 3^{\prime \prime \prime}, 5^{\prime \prime}$ \\
\hline $2^{\prime \prime \prime}$ & $73.3, \mathrm{CH}$ & $3.26, \mathrm{~m}$ & $1 " ', 3 " '$ & & \\
\hline 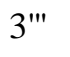 & $75.9, \mathrm{CH}$ & $3.26, \mathrm{~m}$ & 2"', 4"' & & $1^{\prime \prime \prime}$ \\
\hline
\end{tabular}




\begin{tabular}{|c|c|c|c|c|c|}
\hline $4 " '$ & $69.9, \mathrm{CH}$ & $3.15, \mathrm{~m}$ & 3"', 5"' & & \\
\hline $5^{\prime \prime \prime}$ & $77.2, \mathrm{CH}$ & $3.28, \mathrm{~m}$ & 4"', 6"'-Hb & & $1^{\prime \prime \prime}$ \\
\hline \multirow[t]{2}{*}{ 6"' } & $60.8, \mathrm{CH}_{2}$ & 3.71, d (11.2), Ha & 6"'-Hb & $4 " '$ & \\
\hline & & $3.47, \mathrm{dd}(11.9,6.0), \mathrm{Hb}$ & 5"', 6"'-Ha & 4"', 5 "' & \\
\hline
\end{tabular}




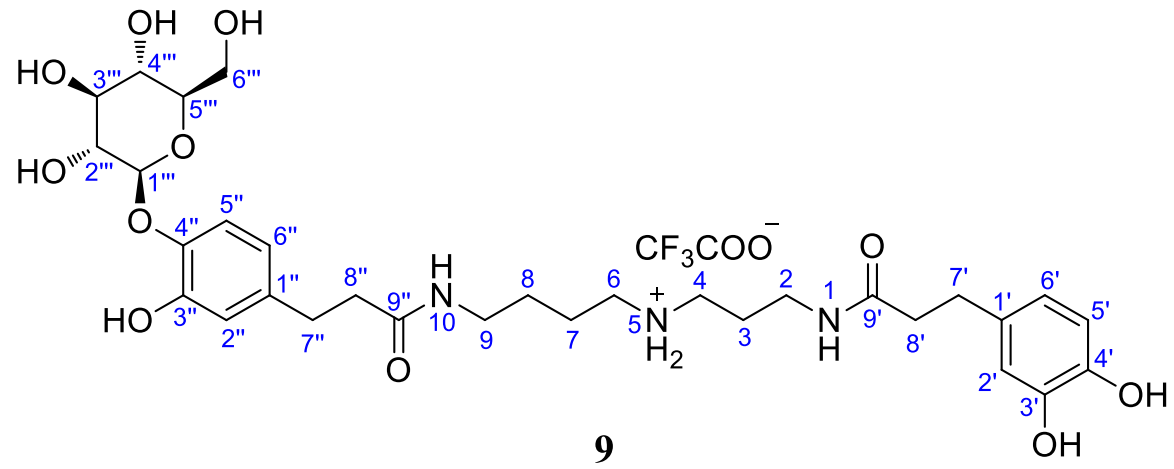

Table S9. NMR (600 MHz for ${ }^{1} \mathrm{H}$ NMR, $150 \mathrm{MHz}$ for ${ }^{13} \mathrm{C}$ NMR, DMSO- $\left.d_{6}\right)$ assignments of 9.

\begin{tabular}{|c|c|c|c|c|c|}
\hline No. & $\delta_{\mathrm{C}}$, type & $\delta_{\mathrm{H}}(J$ in $\mathrm{Hz})$ & ${ }^{1} \mathrm{H}-{ }^{1} \mathrm{H} \mathrm{COSY}$ & HMBC & NOESY \\
\hline 1 & & $8.01 / 7.98, \mathrm{t}(5.8)$ & 2 & $2,9^{\prime}$ & $3,8^{\prime}$ \\
\hline 2 & $35.5, \mathrm{CH}_{2}$ & $3.10, q(6.1)$ & 1,3 & $3,4,9^{\prime}$ & \\
\hline 3 & 26.1, $\mathrm{CH}_{2}$ & 1.68 , quint (6.6) & 2,4 & 2,4 & 1,5 \\
\hline 4 & $44.6, \mathrm{CH}_{2}$ & $2.78, \mathrm{~m}$ & 3,5 & $2,3,6$ & \\
\hline 5 & & 8.37, br s & 4,6 & & 3,7 \\
\hline 6 & 46.6, $\mathrm{CH}_{2}$ & $2.84, \mathrm{~m}$ & 5,7 & $4,7,8$ & \\
\hline 7 & $23.1, \mathrm{CH}_{2}$ & 1.52 , quint (7.1) & 6,8 & $6,8,9$ & 5 \\
\hline 8 & 26.3, $\mathrm{CH}_{2}$ & 1.41, quint (6.9) & 7,9 & $6,7,9$ & 10 \\
\hline 9 & $37.8 / 37.6, \mathrm{CH}_{2}$ & $3.04, \mathrm{q}(6.1)$ & 8,10 & $7,8,9^{\prime \prime}$ & \\
\hline 10 & & 7.86/7.84, t (5.7) & 9 & $9,9^{\prime \prime}$ & $8,8^{\prime \prime}$ \\
\hline $1^{\prime}$ & 132.1/132.0, C & & & & \\
\hline $2^{\prime}$ & $115.7, \mathrm{CH}$ & $6.56, \mathrm{~m}$ & $6^{\prime}$ & $3^{\prime}, 4^{\prime}, 6^{\prime}, 7^{\prime}$ & $7^{\prime}$ \\
\hline $3^{\prime}$ & 145.0, C & & & & \\
\hline $4^{\prime}$ & 143.3, C & & & & \\
\hline $5^{\prime}$ & $115.4, \mathrm{CH}$ & $6.61, \mathrm{~d}(8.0)$ & $6^{\prime}$ & $1^{\prime}, 2^{\prime}, 3^{\prime}, 4^{\prime}, 6^{\prime}$ & \\
\hline $6^{\prime}$ & $118.7, \mathrm{CH}$ & $6.42 / 6.41, \mathrm{dd}(8.0,2.3)$ & $2^{\prime}, 5^{\prime}$ & $2^{\prime}, 3^{\prime}, 4^{\prime}, 7^{\prime}$ & $7^{\prime}$ \\
\hline $7^{\prime}$ & $30.6 / 30.5, \mathrm{CH}_{2}$ & $2.63, \mathrm{t}(7.9)$ & $8^{\prime}$ & $1^{\prime}, 2^{\prime}, 6^{\prime}, 8^{\prime}, 9^{\prime}$ & $2^{\prime}, 6^{\prime}$ \\
\hline $8^{\prime}$ & $37.4 / 37.2, \mathrm{CH}_{2}$ & $2.28, \mathrm{~m}$ & $7^{\prime}$ & $1^{\prime}, 7^{\prime}, 9^{\prime}$ & 1 \\
\hline $9^{\prime}$ & 172.2/172.1, C & & & & \\
\hline $1 "$ & 136.2/136.1, C & & & & \\
\hline $2^{\prime \prime}$ & $115.8, \mathrm{CH}$ & $6.65, \mathrm{~d}(2.2)$ & $6 "$ & 3", 4", 6", 7" & 7", 8" \\
\hline 3" & 146.7, C & & & & \\
\hline 4" & 143.6, C & & & & \\
\hline $5 "$ & $116.9, \mathrm{CH}$ & $6.99, \mathrm{~d}(8.2)$ & $6 "$ & 1", 3", 4", 6" & $1 " '$ \\
\hline 6" & $118.9, \mathrm{CH}$ & $6.55 / 6.54, \mathrm{dd}(8.3,2.3)$ & $2 ", 5 "$ & 2", 4", 7" & 7", 8" \\
\hline 7" & $30.6 / 30.5, \mathrm{CH}_{2}$ & $2.68, \mathrm{t}(7.9)$ & $8 "$ & 1", 2", 6", 8", 9" & 2", 6" \\
\hline $8 "$ & $37.0, \mathrm{CH}_{2}$ & $2.32, \mathrm{~m}$ & 7" & 1", 7", 9" & $10,2 ", 6 "$ \\
\hline 9" & 171.6/171.4, C & & & & \\
\hline $1 " '$ & $102.7, \mathrm{CH}$ & $4.58, \mathrm{~d}(6.8)$ & $2 " '$ & 4", 3"', 4"', 5"' & 5", 3"', 5"' \\
\hline $2 " '$ & $73.3, \mathrm{CH}$ & $3.26, \mathrm{~m}$ & $1^{\prime \prime \prime}, 3^{\prime \prime \prime}$ & & \\
\hline
\end{tabular}




\begin{tabular}{|c|c|c|c|c|c|}
\hline 3"' & $75.9, \mathrm{CH}$ & $3.26, \mathrm{~m}$ & 2"', 4"' & & $1 " '$ \\
\hline $4 " '$ & $69.9, \mathrm{CH}$ & $3.15, \mathrm{~m}$ & 3"', $5^{\prime \prime \prime}$ & & \\
\hline $5^{\prime \prime \prime}$ & $77.2, \mathrm{CH}$ & $3.28, \mathrm{~m}$ & 4"', 6"'-Hb & & $1 " '$ \\
\hline \multirow[t]{2}{*}{ 6"' } & $60.8, \mathrm{CH}_{2}$ & $3.71, \mathrm{~d}(11.2), \mathrm{Ha}$ & 6"'-Hb & 4"' & \\
\hline & & $3.47, \mathrm{dd}(11.9,6.0), \mathrm{Hb}$ & 5"', 6"'-Ha & 4"', 5"' & \\
\hline
\end{tabular}




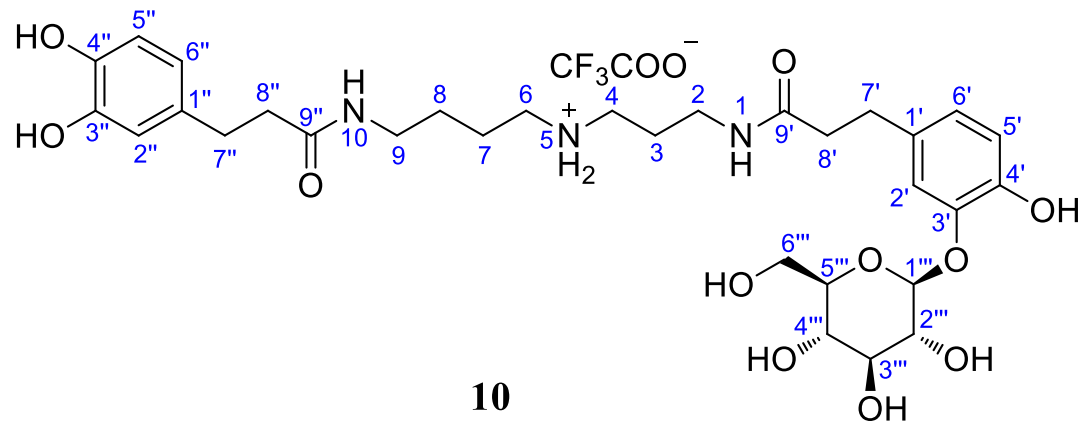

Table S10. NMR (300 MHz for ${ }^{1} \mathrm{H}$ NMR, $75 \mathrm{MHz}$ for ${ }^{13} \mathrm{C}$ NMR, $\mathrm{CD}_{3} \mathrm{OD}$ ) assignments of $\mathbf{1 0 .}$

\begin{tabular}{|c|c|c|c|c|c|}
\hline No. & $\delta_{\mathrm{C}}$, type & $\delta_{\mathrm{H}}(J$ in $\mathrm{Hz})$ & ${ }^{1} \mathrm{H}-{ }^{1} \mathrm{H} \operatorname{COSY}{ }^{a}$ & $\mathrm{HMBC}^{b}$ & ROESY $^{a}$ \\
\hline \multicolumn{6}{|l|}{1} \\
\hline 2 & $36.7, \mathrm{CH}_{2}$ & $3.20, \mathrm{~m}$ & 3 & $3,4,9^{\prime}$ & \\
\hline 3 & $27.5, \mathrm{CH}_{2}$ & 1.71 , quint $(7.0)$ & 2,4 & 2,4 & \\
\hline 4 & $46.1, \mathrm{CH}_{2}$ & $2.58, \mathrm{~m}$ & 3 & 2,3 & \\
\hline \multicolumn{6}{|l|}{5} \\
\hline 6 & $48.6, \mathrm{CH}_{2}$ & $2.79, \mathrm{~m}$ & 7 & $4,7,8$ & 8 \\
\hline 7 & $24.6, \mathrm{CH}_{2}$ & $1.49, \mathrm{~m}$ & 6,8 & $6,8,9$ & 9 \\
\hline 8 & 27.5, $\mathrm{CH}_{2}$ & $1.49, \mathrm{~m}$ & 7,9 & $6,7,9$ & 6 \\
\hline 9 & $39.3, \mathrm{CH}_{2}$ & $3.16, \mathrm{t}(5.9)$ & 8 & $7,8,9^{\prime \prime}$ & 7 \\
\hline \multicolumn{6}{|l|}{10} \\
\hline $1^{\prime}$ & $133.4, \mathrm{C}$ & & & & \\
\hline $2^{\prime}$ & $117.8, \mathrm{CH}$ & 7.04, br s & & $1^{\prime}, 3^{\prime}, 4^{\prime}, 5^{\prime}, 6^{\prime}, 7^{\prime}$ & $7^{\prime}, 8^{\prime}, 1^{\prime \prime \prime}$ \\
\hline $3^{\prime}$ & $146.4, \mathrm{C}$ & & & & \\
\hline $4^{\prime}$ & 146.3, C & & & & \\
\hline $5^{\prime}$ & 117.1, CH & $6.77, \mathrm{~s}$ & & $1^{\prime}, 3^{\prime}, 4^{\prime}$ & \\
\hline $6^{\prime}$ & $124.6, \mathrm{CH}$ & $6.77, \mathrm{~s}$ & & $2^{\prime}, 3^{\prime}, 4^{\prime}, 7^{\prime}$ & $7^{\prime}$ \\
\hline $7^{\prime}$ & $31.8, \mathrm{CH}_{2}$ & $2.84, \mathrm{t}(7.1)$ & $8^{\prime}$ & $1^{\prime}, 2^{\prime}, 6^{\prime}, 8^{\prime}, 9^{\prime}$ & $2^{\prime}, 6^{\prime}$ \\
\hline $8^{\prime}$ & $38.0, \mathrm{CH}_{2}$ & $2.51, \mathrm{~m}$ & $7^{\prime}$ & $1^{\prime}, 7^{\prime}, 9^{\prime}$ & $2^{\prime}$ \\
\hline $9^{\prime}$ & 176.3, C & & & & \\
\hline $1 "$ & 133.7, C & & & & \\
\hline $2^{\prime \prime}$ & $116.8, \mathrm{CH}$ & $6.63, \mathrm{~d}(2.0)$ & $6 "$ & 3", 4", 6", 7" & 7" \\
\hline $3 "$ & 146.1, C & & & & \\
\hline $4 "$ & $144.5, \mathrm{C}$ & & & & \\
\hline $5^{\prime \prime}$ & $116.4, \mathrm{CH}$ & $6.67, \mathrm{~d}(8.0)$ & 6" & 1", 3", 4" & \\
\hline 6" & $120.8, \mathrm{CH}$ & $6.52, \mathrm{dd}(8.0,2.1)$ & $2 ", 5 "$ & 2", 3", 4", 7" & $7^{\prime \prime}$ \\
\hline $7 "$ & $32.4, \mathrm{CH}_{2}$ & $2.76, \mathrm{t}(7.4)$ & $8 "$ & 1", 2", 6", 8", 9" & $2 ", 6 "$ \\
\hline $8 "$ & $39.3, \mathrm{CH}_{2}$ & $2.41, \mathrm{t}(7.5)$ & $7 "$ & 1", 7", 9" & \\
\hline 9" & 175.7, C & & & & \\
\hline $1 " '$ & 103.6, CH & $4.80, \mathrm{~d}(7.8)$ & $2^{\prime \prime \prime}$ & 3', 3"', 4"' & $2^{\prime}, 3^{\prime \prime \prime}, 5^{\prime \prime \prime}$ \\
\hline $2 " '$ & $74.9, \mathrm{CH}$ & $3.47, \mathrm{~m}$ & 1"', 3"' & & \\
\hline 3"' & 77.6, $\mathrm{CH}$ & $3.47, \mathrm{~m}$ & 2"', 4"' & & $1 " '$ \\
\hline
\end{tabular}




$$
\begin{aligned}
& \text { 4"' } \quad 71.3, \mathrm{CH} \quad 3.38, \mathrm{~m} \quad 3 " ', 5 " ' \\
& \text { 5"' } \quad 78.6, \mathrm{CH} \quad 3.47, \mathrm{~m} \quad 4 " ', 6 \text { "'-Ha, 6"'-Hb } \\
& \text { 6"' } \quad 62.4, \mathrm{CH}_{2} \quad 3.97, \mathrm{dd}(12.0,2.0), \mathrm{Ha} \quad 5 \text { "', 6"'-Hb 4"', 5"' } \\
& \text { 3.72, dd (12.0, 5.8), Hb 5"', 6"'-Ha 4"', 5"' }
\end{aligned}
$$

${ }^{a}$ The data were recorded with a Bruker AV 400 spectrometer. ${ }^{b}$ The data were recorded with a Bruker AV 600 spectrometer. 


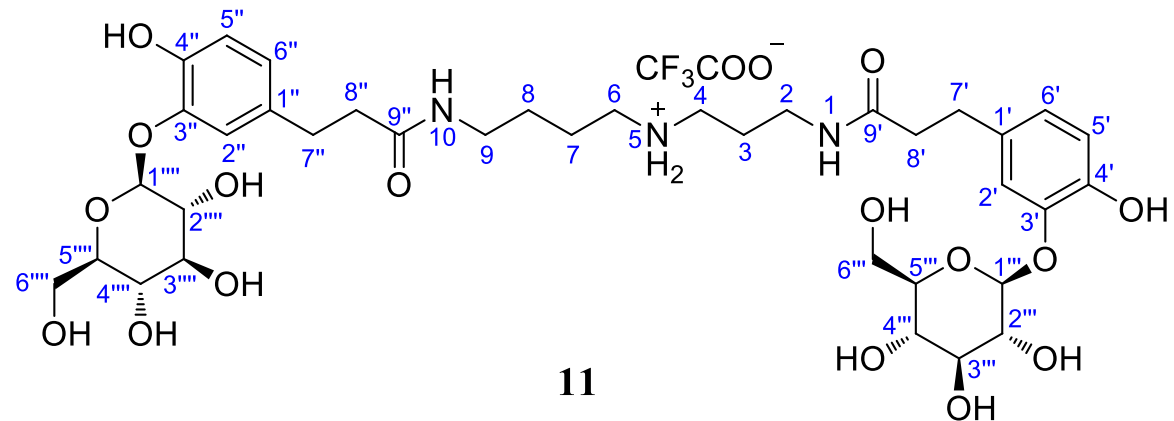

Table S11. NMR (600 MHz for ${ }^{1} \mathrm{H}$ NMR, $100 \mathrm{MHz}$ for ${ }^{13} \mathrm{C}$ NMR, DMSO- $d_{6}$ ) assignments of 11.

\begin{tabular}{|c|c|c|c|c|c|}
\hline No. & $\delta_{\mathrm{C}}$, type $^{a}$ & $\delta_{\mathrm{H}}(J$ in $\mathrm{Hz})$ & ${ }^{1} \mathrm{H}-{ }^{1} \mathrm{H} \operatorname{COS} Y^{a}$ & HMBC & $\mathrm{ROESY}^{a}$ \\
\hline 1 & & $7.97, \mathrm{t}(5.8)$ & 2 & $2,3,9^{\prime}$ & $3,8^{\prime}$ \\
\hline 2 & $35.5, \mathrm{CH}_{2}$ & $3.09, \mathrm{q}(6.4)$ & 1,3 & $3,4,9^{\prime}$ & 4 \\
\hline 3 & 26.0, $\mathrm{CH}_{2}$ & 1.67 , quint (6.8) & 2,4 & 2,4 & 1,5 \\
\hline 4 & 44.6, $\mathrm{CH}_{2}$ & $2.76, \mathrm{~m}$ & 3,5 & $2,3,6$ & 2 \\
\hline 5 & & 8.36 , br s & 4,6 & 4,6 & 3,7 \\
\hline 6 & $46.6, \mathrm{CH}_{2}$ & $2.82, \mathrm{~m}$ & 5,7 & $4,7,8$ & 8 \\
\hline 7 & $23.1, \mathrm{CH}_{2}$ & 1.50, quint $(7.0)$ & 6,8 & $6,8,9$ & 5,9 \\
\hline 8 & $26.2, \mathrm{CH}_{2}$ & 1.40, quint (6.9) & 7,9 & $6,7,9$ & 6,10 \\
\hline 9 & $37.8, \mathrm{CH}_{2}$ & $3.04, q(6.6)$ & 8,10 & $7,8,9^{\prime \prime}$ & 7 \\
\hline 10 & & $7.83, \mathrm{t}(5.6)$ & 9 & $8,9,9^{\prime \prime}$ & $8,8^{\prime \prime}$ \\
\hline $1^{\prime}$ & 132.0, C & & & & \\
\hline $2^{\prime}$ & $116.8^{* 1}, \mathrm{CH}$ & $6.95, \mathrm{~m}$ & $6^{\prime}, 7^{\prime}$ & $1^{\prime}, 3^{\prime}, 4^{\prime}, 5^{\prime}, 6^{\prime}, 7^{\prime}$ & $1^{\prime \prime}, 7^{\prime}, 8^{\prime}$ \\
\hline $3^{\prime}$ & 145.1, C & & & & \\
\hline $4^{\prime}$ & $144.9, \mathrm{C}$ & & & & \\
\hline $5^{\prime}$ & $115.6, \mathrm{CH}$ & $6.70, d(8.1)$ & $6^{\prime}$ & $1^{\prime}, 2^{\prime}, 3^{\prime}, 4^{\prime}, 6^{\prime}$ & \\
\hline $6^{\prime}$ & $122.5, \mathrm{CH}$ & $6.68, \mathrm{dd}(8.2,1.9)$ & $2^{\prime}, 5^{\prime}$ & $2^{\prime}, 3^{\prime}, 4^{\prime}, 7^{\prime}$ & $7^{\prime}, 8^{\prime}$ \\
\hline $7^{\prime}$ & $30.5, \mathrm{CH}_{2}$ & $2.70, \mathrm{~m}$ & $2^{\prime}, 8^{\prime}$ & $1^{\prime}, 2^{\prime}, 6^{\prime}, 8^{\prime}, 9^{\prime}$ & $2^{\prime}, 6^{\prime}$ \\
\hline $8^{\prime}$ & $36.9, \mathrm{CH}_{2}$ & $2.34, \mathrm{t}(7.7)$ & $7^{\prime}$ & $1^{\prime}, 7^{\prime}, 9^{\prime}$ & $1,2^{\prime}, 6^{\prime}$ \\
\hline $9^{\prime}$ & 172.1, C & & & & \\
\hline $1 "$ & 132.2, C & & & & \\
\hline $2 "$ & $116.9^{* 1}, \mathrm{CH}$ & $6.95, \mathrm{~m}$ & 6", 7" & 1", 3", 4", 5", 6", 7" & $1 " ',, 7 ", 8 "$ \\
\hline $3 "$ & 145.1, C & & & & \\
\hline $4 "$ & $144.9, \mathrm{C}$ & & & & \\
\hline $5^{\prime \prime}$ & $115.6, \mathrm{CH}$ & $6.70, \mathrm{~d}(8.1)$ & 6" & 1", 2", 3", 4", 6" & \\
\hline $6 "$ & $122.5, \mathrm{CH}$ & $6.68, \mathrm{dd}(8.2,1.9)$ & $2 ", 5 "$ & 2", 3", 4", 7" & 7", 8" \\
\hline 7" & $30.6, \mathrm{CH}_{2}$ & $2.67, \mathrm{~m}$ & $2 ", 8 "$ & 1", 2", 6", 8", 9" & 2", 6" \\
\hline $8 "$ & $37.2, \mathrm{CH}_{2}$ & $2.31, \mathrm{t}(7.8)$ & 7" & 1", 7", 9" & 10, 2", 6" \\
\hline 9" & 171.5, C & & & & \\
\hline $1 " '$ & $102.4, \mathrm{CH}$ & $4.63, \mathrm{~d}(7.3)^{* 2}$ & $2^{\prime \prime \prime}$ & 3', 3"', 4"', 5"' & 2', 3"', 5"' \\
\hline $2^{\prime \prime \prime}$ & 73.3, $\mathrm{CH}$ & $3.27, \mathrm{~m}$ & 1"', 3 "' & & \\
\hline $3^{\prime \prime \prime}$ & 75.9, $\mathrm{CH}$ & $3.27, \mathrm{~m}$ & 2"', 4"' & & $1 " '$ \\
\hline
\end{tabular}




\begin{tabular}{|c|c|c|c|c|c|}
\hline 4"' & $69.8, \mathrm{CH}$ & $3.16, \mathrm{~m}$ & 3"', $5^{\prime \prime \prime}$ & & \\
\hline $5 " '$ & $77.3, \mathrm{CH}$ & $3.31, \mathrm{~m}$ & 4"', 6'"-Ha, 6"'-Hb & & $1 " '$ \\
\hline \multirow[t]{2}{*}{ 6"' } & $60.8, \mathrm{CH}_{2}$ & $3.74, \mathrm{dd}(11.8,1.7), \mathrm{Ha}^{* 3}$ & 5"', 6"'-Hb & $4 " ', 5 " '$ & \\
\hline & & $3.49, \mathrm{~m}, \mathrm{Hb}$ & 5"', 6"'-На & 4'", 5"' & \\
\hline $1 "$ "' & $102.4, \mathrm{CH}$ & $4.62, \mathrm{~d}(7.3)^{* 2}$ & $2 " '$ & 3", 3'"', 4"'", 5"'" & 2", 3 "', 5 "'" \\
\hline $2 " '$ & $73.3, \mathrm{CH}$ & $3.27, \mathrm{~m}$ & 1"'", 3"'" & & \\
\hline 3"'" & $75.9, \mathrm{CH}$ & $3.27, \mathrm{~m}$ & 2"'", 4"'" & & $1 " '+$ \\
\hline 4"'" & $69.8, \mathrm{CH}$ & $3.16, \mathrm{~m}$ & 3"'", 5"'" & & \\
\hline $5^{\prime \prime \prime}$ & $77.3, \mathrm{CH}$ & $3.31, \mathrm{~m}$ & 4"'', 6"'--Ha, 6"''-Hb & & $1 " '+$ \\
\hline \multirow[t]{2}{*}{ 6"' } & $60.8, \mathrm{CH}_{2}$ & $3.74, \mathrm{dd}(11.9,1.9), \mathrm{Ha}^{* 3}$ & $5^{\prime \prime \prime}, 6$ 6"'-Hb & $4^{\prime \prime \prime}, 5^{\prime \prime \prime}$ & \\
\hline & & $3.49, \mathrm{~m}, \mathrm{Hb}$ & 5"', 6 "'"-Ha & 4'"', 5 "'" & \\
\hline
\end{tabular}

${ }^{a}$ The data were recorded with a Bruker AV 400 spectrometer. * Assignment may be interchanged. 


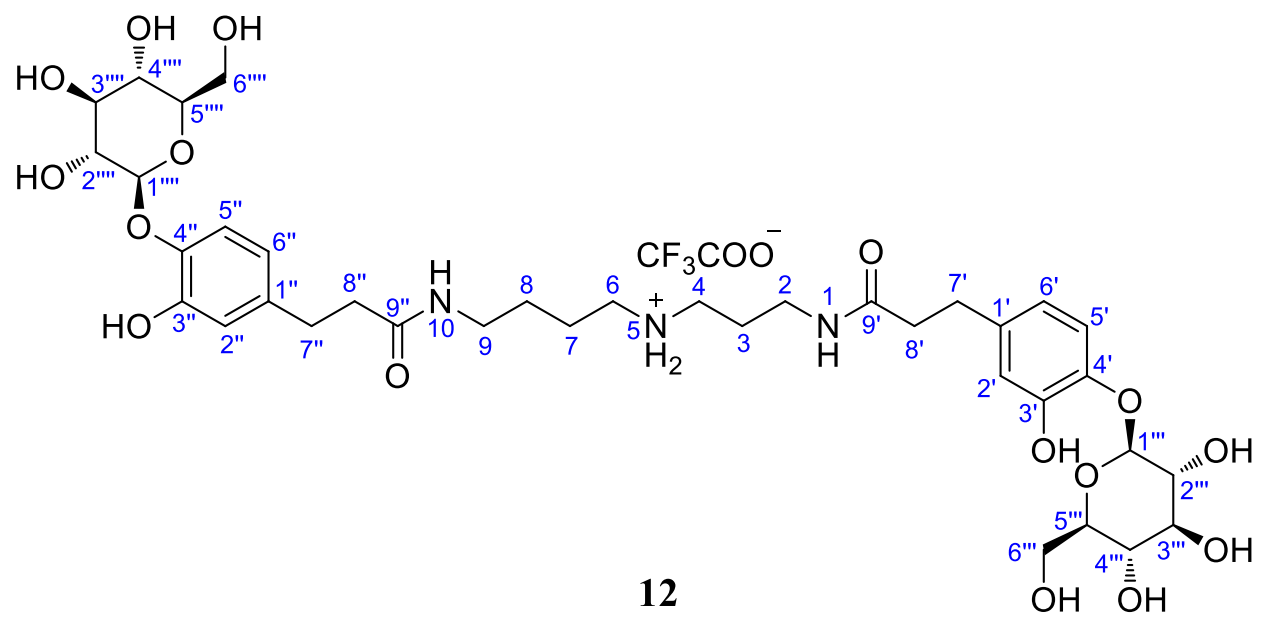

Table S12. NMR (600 MHz for ${ }^{1} \mathrm{H}$ NMR, $100 \mathrm{MHz}$ for ${ }^{13} \mathrm{C}$ NMR, DMSO- $d_{6}$ ) assignments of 12.

\begin{tabular}{|c|c|c|c|c|c|}
\hline No. & $\delta_{\mathrm{C}}$, type $^{a}$ & $\delta_{\mathrm{H}}(J$ in $\mathrm{Hz})$ & ${ }^{1} \mathrm{H}-{ }^{1} \mathrm{H} \cos Y^{a}$ & HMBC & ROESY $^{a}$ \\
\hline 1 & & $8.01, \mathrm{t}(5.8)$ & 2 & $2,9^{\prime}$ & $3,8^{\prime}$ \\
\hline 2 & $35.5, \mathrm{CH}_{2}$ & $3.10, q(6.2)$ & 1,3 & $3,4,9^{\prime}$ & \\
\hline 3 & $26.1, \mathrm{CH}_{2}$ & 1.68 , quint (7.4) & 2,4 & 2,4 & 1,5 \\
\hline 4 & $44.6, \mathrm{CH}_{2}$ & $2.79, \mathrm{~m}$ & 3,5 & $2,3,6$ & \\
\hline 5 & & 8.40 , br s & 4,6 & 4,6 & 3,7 \\
\hline 6 & $46.6, \mathrm{CH}_{2}$ & $2.85, \mathrm{~m}$ & 5,7 & $4,7,8$ & \\
\hline 7 & $23.1, \mathrm{CH}_{2}$ & 1.52 , quint (7.1) & 6,8 & $6,8,9$ & 5 \\
\hline 8 & $26.2, \mathrm{CH}_{2}$ & 1.41 , quint (7.5) & 7,9 & $6,7,9$ & 10 \\
\hline 9 & $37.8, \mathrm{CH}_{2}$ & $3.04, \mathrm{q}(6.1)$ & 8,10 & $7,8,9^{\prime \prime}$ & \\
\hline 10 & & $7.86, \mathrm{t}(5.6)$ & 9 & $9,9^{\prime \prime}$ & $8,8^{\prime \prime}$ \\
\hline $1^{\prime}$ & 136.2, C & & & & \\
\hline $2^{\prime}$ & $115.8, \mathrm{CH}$ & $6.65, \mathrm{~d}(2.2)$ & $6^{\prime}$ & $3^{\prime}, 4^{\prime}, 5^{\prime}, 6^{\prime}, 7^{\prime}$ & $7^{\prime}, 8^{\prime}$ \\
\hline $3^{\prime}$ & $146.9, \mathrm{C}$ & & & & \\
\hline $4^{\prime}$ & 143.6, C & & & & \\
\hline $5^{\prime}$ & 117.0, CH & $6.99, \mathrm{~d}(8.2)$ & $6^{\prime}$ & $1^{\prime}, 2^{\prime}, 3^{\prime}, 4^{\prime}$ & 1 1" \\
\hline $6^{\prime}$ & $118.9, \mathrm{CH}$ & $6.55, \mathrm{dd}(8.2,2.4)$ & $2^{\prime}, 5^{\prime}$ & $2^{\prime}, 3^{\prime}, 4^{\prime}, 7^{\prime}$ & $7^{\prime}, 8^{\prime}$ \\
\hline $7^{\prime}$ & $30.6^{*}, \mathrm{CH}_{2}$ & $2.69, \mathrm{t}(7.5)$ & $8^{\prime}$ & $1^{\prime}, 2^{\prime}, 6^{\prime}, 8^{\prime}, 9^{\prime}$ & $2^{\prime}, 6^{\prime}$ \\
\hline $8^{\prime}$ & $37.0, \mathrm{CH}_{2}$ & $2.33, \mathrm{t}(7.5)$ & $7^{\prime}$ & $1^{\prime}, 7^{\prime}, 9^{\prime}$ & $1,2^{\prime}, 6^{\prime}$ \\
\hline $9^{\prime}$ & 172.0, C & & & & \\
\hline $1 "$ & 136.2, C & & & & \\
\hline $2^{\prime \prime}$ & $115.8, \mathrm{CH}$ & $6.65, \mathrm{~d}(2.2)$ & $6 "$ & $3^{\prime}, 4^{\prime}, 5^{\prime}, 6^{\prime}, 7^{\prime}$ & 7", 8" \\
\hline $3 "$ & 146.9, C & & & & \\
\hline $4 "$ & 143.6, C & & & & \\
\hline $5^{\prime \prime}$ & 117.0, CH & $6.99, \mathrm{~d}(8.2)$ & 6" & $1^{\prime}, 2^{\prime}, 3^{\prime}, 4^{\prime}$ & $1^{\prime \prime \prime \prime}$ \\
\hline 6" & $118.9, \mathrm{CH}$ & $6.55, \mathrm{dd}(8.2,2.4)$ & $2 ", 5 "$ & $2^{\prime}, 3^{\prime}, 4^{\prime}, 7^{\prime}$ & 7", 8" \\
\hline $7 "$ & $30.5^{*}, \mathrm{CH}_{2}$ & $2.68, \mathrm{t}(7.9)$ & $8 "$ & $1^{\prime}, 2^{\prime}, 6^{\prime}, 8^{\prime}, 9^{\prime}$ & 2", 6" \\
\hline $8^{\prime \prime}$ & $37.2, \mathrm{CH}_{2}$ & $2.30, \mathrm{t}(7.4)$ & 7" & $1^{\prime}, 7^{\prime}, 9^{\prime}$ & $10,2^{\prime \prime}, 6^{\prime \prime}$ \\
\hline
\end{tabular}




\begin{tabular}{|c|c|c|c|c|c|}
\hline 9" & $171.4, \mathrm{C}$ & & & & \\
\hline $1 " '$ & $102.7, \mathrm{CH}$ & $4.58, \mathrm{~d}(6.6)$ & $2^{\prime \prime \prime}$ & 4', 3"', 4"', 5"' & 5', 3'", $5^{\prime \prime \prime}$ \\
\hline $2^{\prime \prime \prime}$ & $73.3, \mathrm{CH}$ & $3.27, \mathrm{~m}$ & $1 " ', 3 " '$ & & \\
\hline 3"' & $75.9, \mathrm{CH}$ & $3.27, \mathrm{~m}$ & 2"', 4"' & & 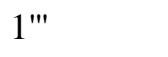 \\
\hline 4"' & $69.9, \mathrm{CH}$ & $3.15, \mathrm{~m}$ & 3"', $5 " '$ & & \\
\hline $5 " '$ & $77.2, \mathrm{CH}$ & $3.27, \mathrm{~m}$ & 4"', 6"'-Hb & & 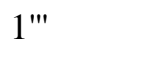 \\
\hline \multirow[t]{2}{*}{ 6"' } & $60.8, \mathrm{CH}_{2}$ & $3.71, \mathrm{~d}(11.2), \mathrm{Ha}$ & 6"'-Hb & 4"', 5"' & \\
\hline & & $3.47, \mathrm{dd}(11.8,5.9), \mathrm{Hb}$ & 5"', 6"'-Ha & 4"', 5"' & \\
\hline 1 '"' & $102.7, \mathrm{CH}$ & $4.58, \mathrm{~d}(6.6)$ & $2^{\prime \prime \prime \prime}$ & 4", $3^{\prime \prime \prime \prime}, 4 " ', 5$,"' & $5^{\prime \prime}, 3^{\prime \prime \prime}, 5^{\prime \prime \prime}$ \\
\hline $2^{\prime \prime \prime \prime}$ & $73.3, \mathrm{CH}$ & $3.27, \mathrm{~m}$ & 1"'", $3^{\prime \prime " '}$ & & \\
\hline $3^{\prime \prime \prime \prime}$ & $75.9, \mathrm{CH}$ & $3.27, \mathrm{~m}$ & 2"'", 4'"' & & $1^{\prime \prime \prime \prime}$ \\
\hline 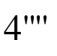 & $69.9, \mathrm{CH}$ & $3.15, \mathrm{~m}$ & $3^{\prime \prime \prime \prime}, 5^{\prime \prime \prime \prime}$ & & \\
\hline $5^{\prime \prime \prime}$ & 77.2, $\mathrm{CH}$ & $3.27, \mathrm{~m}$ & 4"'", 6'"'-Hb & & $1^{\prime \prime \prime \prime}$ \\
\hline \multirow[t]{2}{*}{ 6"' } & $60.8, \mathrm{CH}_{2}$ & 3.71, d (11.2), Ha & 6"''-Hb & 4"'", 5"'" & \\
\hline & & $3.47, \mathrm{dd}(11.8,5.9), \mathrm{Hb}$ & 5"'", 6'"'-Нa & 4"'", 5"'" & \\
\hline
\end{tabular}

${ }^{a}$ The data were recorded with a Bruker AV 400 spectrometer. *Assignment may be interchanged. 


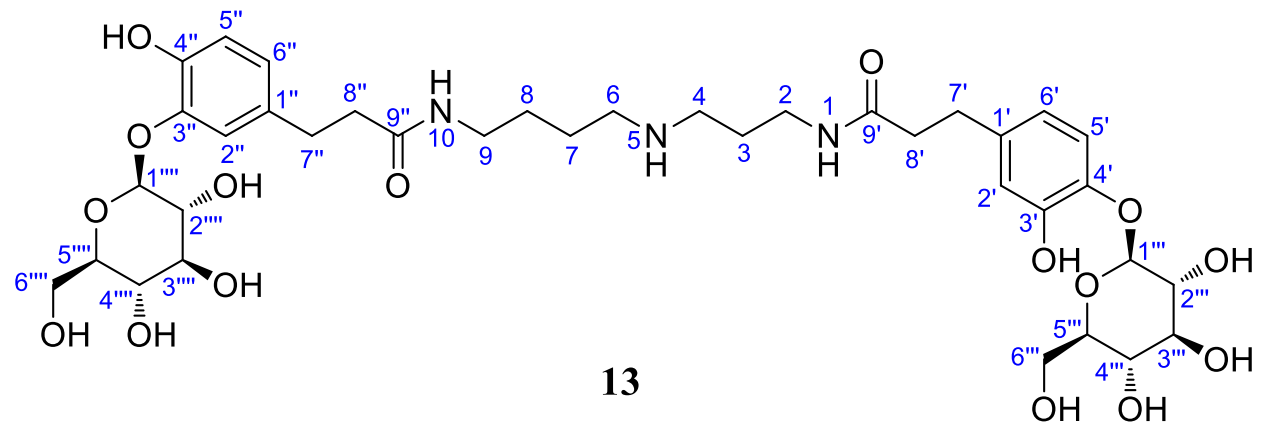

Table S13. NMR (600 MHz for ${ }^{1} \mathrm{H}$ NMR, $150 \mathrm{MHz}$ for ${ }^{13} \mathrm{C}$ NMR, DMSO- $d_{6}$ ) assignments of 13.

\begin{tabular}{|c|c|c|c|c|c|}
\hline No. & $\delta_{\mathrm{C}}$, type & $\delta_{\mathrm{H}}(J$ in $\mathrm{Hz})$ & ${ }^{1} \mathrm{H}-{ }^{1} \mathrm{H}$ COSY & HMBC & NOESY \\
\hline 1 & & $7.79, \mathrm{t}(5.6)$ & 2 & $2,9^{\prime}$ & $8^{\prime}$ \\
\hline 2 & $36.8, \mathrm{CH}_{2}$ & $3.04, \mathrm{q}(5.9)$ & 1,3 & $3,4,9^{\prime}$ & \\
\hline 3 & $29.5, \mathrm{CH}_{2}$ & 1.48 , quint (6.8) & 2,4 & 2,4 & \\
\hline 4 & $46.9, \mathrm{CH}_{2}$ & $2.42, \mathrm{t}(7.0)$ & 3 & $2,3,6$ & \\
\hline 5 & & & & & \\
\hline 6 & $49.0, \mathrm{CH}_{2}$ & $2.41, \mathrm{t}(7.0)$ & 7 & $4,7,8$ & \\
\hline 7 & 27.0, $\mathrm{CH}_{2}$ & 1.30 , quint (6.8) & 6,8 & $6,8,9$ & \\
\hline 8 & 27.1, $\mathrm{CH}_{2}$ & 1.36 , quint (6.6) & 7,9 & $6,7,9$ & \\
\hline 9 & $38.5, \mathrm{CH}_{2}$ & $2.99, \mathrm{q}(6.1)$ & 8,10 & $7,8,9^{\prime \prime}$ & \\
\hline 10 & & $7.74, \mathrm{t}(5.5)$ & 9 & $9,9^{\prime \prime}$ & $8 "$ \\
\hline $1^{\prime}$ & $136.4, \mathrm{C}$ & & & & \\
\hline $2^{\prime}$ & $116.8, \mathrm{CH}$ & 6.55, br d (2.0) & $6^{\prime}, 7^{\prime}$ & $3^{\prime}, 4^{\prime}, 6^{\prime}, 7^{\prime}$ & $7^{\prime}, 8^{\prime}$ \\
\hline $3^{\prime}$ & $150.2, \mathrm{C}$ & & & & \\
\hline $4^{\prime}$ & $144.5, \mathrm{C}$ & & & & \\
\hline $5^{\prime}$ & $117.8, \mathrm{CH}$ & $6.91, \mathrm{~d}(8.2)$ & $6^{\prime}$ & $1^{\prime}, 2^{\prime}, 3^{\prime}, 4^{\prime}$ & $1 " '$ \\
\hline $6^{\prime}$ & $116.5, \mathrm{CH}$ & $6.37, \mathrm{dd}(8.2,1.7)$ & $2^{\prime}, 5^{\prime}$ & $2^{\prime}, 4^{\prime}, 7^{\prime}$ & $7^{\prime}$ \\
\hline $7^{\prime}$ & $30.9, \mathrm{CH}_{2}$ & $2.63, \mathrm{~m}$ & $2^{\prime}, 8^{\prime}$ & $1^{\prime}, 2^{\prime}, 6^{\prime}, 8^{\prime}, 9^{\prime}$ & $2^{\prime}, 6^{\prime}$ \\
\hline $8^{\prime}$ & $37.4, \mathrm{CH}_{2}$ & $2.27, \mathrm{t}(7.3)$ & $7^{\prime}$ & $1^{\prime}, 7^{\prime}, 9^{\prime}$ & $1,2^{\prime}$ \\
\hline $9^{\prime}$ & 171.5, C & & & & \\
\hline $1 "$ & $130.4, \mathrm{C}$ & & & & \\
\hline $2^{\prime \prime}$ & $117.4, \mathrm{CH}$ & 6.90, br s & 6", 7" & 3", 4", 6", 7" & $1 " ',, 7 ", 8 "$ \\
\hline $3 "$ & 145.7, C & & & & \\
\hline $4 "$ & 147.3, C & & & & \\
\hline $5^{\prime \prime}$ & $116.3, \mathrm{CH}$ & $6.62, \mathrm{~s}$ & & 1", 2", 3", 4" & \\
\hline $6 "$ & $122.7, \mathrm{CH}$ & $6.62, \mathrm{~s}$ & $2^{\prime \prime}$ & 2", 4", 7" & 7", 8" \\
\hline 7" & $30.6, \mathrm{CH}_{2}$ & $2.65, \mathrm{~m}$ & 2", 8" & 1", 2", 6", 8", 9" & 2", 6" \\
\hline $8^{\prime \prime}$ & $37.3, \mathrm{CH}_{2}$ & $2.28, \mathrm{t}(7.5)$ & 7" & 1", 7", 9" & 10, 2", 6" \\
\hline 9" & 171.4, C & & & & \\
\hline $1 " '$ & 103.6, CH & $4.52, \mathrm{~d}(7.3)$ & $2^{\prime \prime \prime}$ & $4^{\prime}, 3^{\prime \prime \prime}$ & $5^{\prime}, 3^{\prime \prime}, 5^{\prime \prime \prime}$ \\
\hline $2^{\prime \prime \prime}$ & $73.5^{* 1}, \mathrm{CH}$ & $3.25, \mathrm{~m}$ & 1"', $3 " '$ & & \\
\hline $3 " '$ & $76.2, \mathrm{CH}$ & $3.25, \mathrm{~m}$ & 2"', 4"' & & $1 " '$ \\
\hline
\end{tabular}




\begin{tabular}{|c|c|c|c|c|c|}
\hline $4 " '$ & $69.9, \mathrm{CH}$ & $3.15, \mathrm{~m}$ & 3"', $5^{\prime \prime \prime}$ & & \\
\hline $5^{\prime \prime}$ & $77.3, \mathrm{CH}$ & $3.25, \mathrm{~m}$ & 4"', 6"'-Ha, 6"'-Hb & & $1 " '$ \\
\hline \multirow[t]{2}{*}{ 6"' } & $60.9, \mathrm{CH}_{2}$ & $3.70, \mathrm{dd}(11.5,1.3), \mathrm{Ha}^{* 2}$ & 5"', 6"'-Hb & 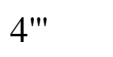 & \\
\hline & & 3.47, dd $(12.4,5.9), \mathrm{Hb}^{* 3}$ & 5"', 6"'-Ha & $5^{\prime \prime \prime}$ & \\
\hline $1^{\prime \prime \prime}$ & $103.1, \mathrm{CH}$ & $4.58, \mathrm{~d}(7.4)$ & $2^{\prime \prime \prime}$ & 3", $3^{\prime \prime \prime}$ & 2", $3 "$ "', $5^{\prime \prime \prime}$ \\
\hline $2^{\prime \prime \prime}$ & $73.4^{* 1}, \mathrm{CH}$ & $3.25, \mathrm{~m}$ & $1^{\prime \prime \prime}, 3^{\prime \prime \prime}$ & & \\
\hline $3^{\prime \prime \prime}$ & $76.2, \mathrm{CH}$ & $3.25, \mathrm{~m}$ & 2"'", 4"'" & & 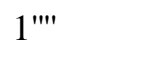 \\
\hline $4^{\prime \prime \prime}$ & $69.9, \mathrm{CH}$ & $3.15, \mathrm{~m}$ & 3"', 5 "'" & & \\
\hline $5^{\prime \prime \prime}$ & $77.3, \mathrm{CH}$ & $3.25, \mathrm{~m}$ & 4"'", 6"'"-Ha, 6"'"-Hb & & $1^{\prime \prime \prime \prime}$ \\
\hline \multirow[t]{2}{*}{ 6"'" } & $60.9, \mathrm{CH}_{2}$ & $3.73, \mathrm{dd}(11.6,1.4), \mathrm{Ha}^{* 2}$ & $5^{\prime \prime \prime}, 6$ "'"-Hb & $4^{\prime \prime \prime \prime}$ & \\
\hline & & $3.49, \mathrm{dd}(12.3,5.9), \mathrm{Hb}^{* 3}$ & 5"'", 6"'"-Ha & $5^{\prime \prime \prime \prime}$ & \\
\hline
\end{tabular}

*Assignment may be interchanged. 


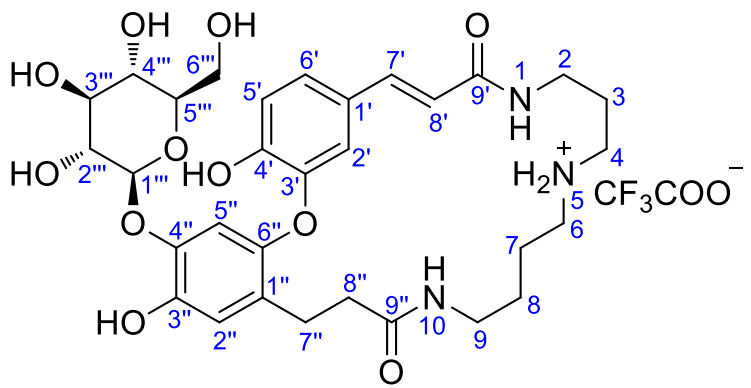

14

Table S14. NMR (600 MHz for ${ }^{1} \mathrm{H}$ NMR, $150 \mathrm{MHz}$ for ${ }^{13} \mathrm{C}$ NMR, DMSO- $d_{6}$ ) assignments of 14.

\begin{tabular}{|c|c|c|c|c|c|}
\hline No. & $\delta_{\mathrm{C}}$, type & $\delta_{\mathrm{H}}(J$ in $\mathrm{Hz})$ & ${ }^{1} \mathrm{H}-{ }^{1} \mathrm{H}$ COSY & HMBC & NOESY \\
\hline 1 & & $8.17, \mathrm{t}(5.6)$ & 2 & $2,8^{\prime}, 9^{\prime}$ & $3,4,8^{\prime}$ \\
\hline 2 & $35.3, \mathrm{CH}_{2}$ & $3.25, \mathrm{~m}$ & 1,3 & $3,4,9^{\prime}$ & \\
\hline 3 & $25.4, \mathrm{CH}_{2}$ & 1.73 , quint (6.0) & 2,4 & 2,4 & 1 \\
\hline 4 & $42.9, \mathrm{CH}_{2}$ & $2.84, \mathrm{~m}$ & 3,5 & 2,3 & 1 \\
\hline 5 & & 8.44 , br s & 4,6 & & \\
\hline 6 & $45.4, \mathrm{CH}_{2}$ & $2.86, \mathrm{~m}$ & 5,7 & $4,7,8$ & 8 \\
\hline 7 & $22.2, \mathrm{CH}_{2}$ & 1.51 , quint (6.8) & 6,8 & $6,8,9$ & 9,10 \\
\hline 8 & $25.8, \mathrm{CH}_{2}$ & 1.38 , quint (7.4) & 7,9 & $6,7,9$ & 6,10 \\
\hline 9 & $38.1, \mathrm{CH}_{2}$ & $3.03, \mathrm{q}(5.9)$ & 8,10 & $7,8,9^{\prime \prime}$ & 7 \\
\hline 10 & & $7.68, \mathrm{t}(5.2)$ & 9 & $8,9,9^{\prime \prime}$ & $7,8,8^{\prime \prime}$ \\
\hline $1^{\prime}$ & 126.3, C & & & & \\
\hline $2^{\prime}$ & $115.2, \mathrm{CH}$ & $6.84, \mathrm{~d}(1.8)$ & $6^{\prime}$ & $1^{\prime}, 3^{\prime}, 4^{\prime}, 5^{\prime}, 6^{\prime}, 7^{\prime}$ & $7^{\prime}, 8^{\prime}$ \\
\hline $3^{\prime}$ & 147.1, C & & & & \\
\hline $4^{\prime}$ & 149.0, C & & & & \\
\hline $5^{\prime}$ & $116.7, \mathrm{CH}$ & $6.90, \mathrm{~d}(8.2)$ & $6^{\prime}$ & $1^{\prime}, 2^{\prime}, 3^{\prime}, 4^{\prime}, 6^{\prime}$ & $4^{\prime}-\mathrm{OH}$ \\
\hline $6^{\prime}$ & 123.7, CH & $7.08, \mathrm{dd}(8.3,1.7)$ & $2^{\prime}, 5^{\prime}$ & $2^{\prime}, 3^{\prime}, 4^{\prime}, 5^{\prime}, 7^{\prime}$ & $7^{\prime}, 8^{\prime}$ \\
\hline $7^{\prime}$ & 139.1, CH & $7.26, \mathrm{~d}(15.7)$ & $8^{\prime}$ & $1^{\prime}, 2^{\prime}, 6^{\prime}, 8^{\prime}, 9^{\prime}$ & $2^{\prime}, 6^{\prime}$ \\
\hline $8^{\prime}$ & $118.7, \mathrm{CH}$ & $6.21, \mathrm{~d}(15.8)$ & $7^{\prime}$ & $1^{\prime}, 7^{\prime}, 9^{\prime}$ & $1,2^{\prime}, 6^{\prime}$ \\
\hline $9^{\prime}$ & 166.2, C & & & & \\
\hline $1 "$ & 127.6, C & & & & \\
\hline $2^{\prime \prime}$ & $116.8, \mathrm{CH}$ & 6.82, br s & 7" & 1", 3", 4", 5", 6", 7" & 3"-OH, 7", 8" \\
\hline $3 "$ & 143.6, C & & & & \\
\hline $4 "$ & 144.0, C & & & & \\
\hline $5^{\prime \prime}$ & $109.8, \mathrm{CH}$ & $6.68, \mathrm{~s}$ & & 1", 3", 4", 6", 7" & $1 " '$ \\
\hline $6 "$ & 145.3, C & & & & \\
\hline $7 "$ & $23.3, \mathrm{CH}_{2}$ & $2.70, \mathrm{~m}$ & $2 ", 8^{\prime \prime}$ & 1", 2", 6", 8", 9" & $2 "$ \\
\hline $8^{\prime \prime}$ & $35.2, \mathrm{CH}_{2}$ & $2.33, \mathrm{t}(7.0)$ & 7" & 1", 7", 9" & $10,2^{\prime \prime}$ \\
\hline $9 "$ & 171.2, C & & & & \\
\hline $1 " '$ & $102.3, \mathrm{CH}$ & $4.59, \mathrm{~d}(7.5)$ & $2 " '$ & 4", $3 " ', 5^{\prime \prime \prime}$ & $5^{\prime \prime}, 3^{\prime \prime \prime}, 5^{\prime \prime \prime}$ \\
\hline $2^{\prime \prime \prime}$ & $73.2, \mathrm{CH}$ & $3.23, \mathrm{~m}$ & 1"', 3"', 2"'-OH & & \\
\hline
\end{tabular}




\begin{tabular}{|c|c|c|c|c|c|}
\hline 3"' & $75.8, \mathrm{CH}$ & $3.23, \mathrm{~m}$ & 2"', 4"', 3"'-OH & & $1^{\prime \prime \prime}$ \\
\hline 4"' & $69.4, \mathrm{CH}$ & $3.17, \mathrm{~m}$ & 3"', 5"', 4"'-OH & & \\
\hline $5 " '$ & 76.9, $\mathrm{CH}$ & $3.17, \mathrm{~m}$ & 4"', 6"'-Ha, 6"'-Hb & & $1 " '$ \\
\hline \multirow[t]{2}{*}{ 6"' } & $60.3, \mathrm{CH}_{2}$ & $3.53, \mathrm{~m}, \mathrm{Ha}$ & 5"', 6"'-Hb, 6"'-OH & 4"', 5"' & \\
\hline & & $3.44, \mathrm{~m}, \mathrm{Hb}$ & 5"', 6"'-Ha, 6"'-OH & 4"', 5"' & \\
\hline 4'-OH & & $9.88, \mathrm{~s}$ & & $3^{\prime}, 4^{\prime}, 5^{\prime}$ & $5^{\prime}$ \\
\hline 3"-OH & & $8.52, \mathrm{~s}$ & & 1", 2", 3", 4" & $2^{\prime \prime}$ \\
\hline 2"'-OH & & 5.46, br s $^{*}$ & $2^{\prime \prime \prime}$ & 2"', 3"' & \\
\hline 3"'-OH & & 5.06, br s$^{*}$ & $3 " '$ & 2"', $3 " '$ & \\
\hline 4"'-OH & & 4.98, br s & $4 " '$ & 4"', 5"' & \\
\hline 6"'-OH & & $4.44, \mathrm{t}(5.2)$ & 6"'-Ha, 6"'-Hb & 5"', 6"' & \\
\hline
\end{tabular}

*Assignment may be interchanged. 


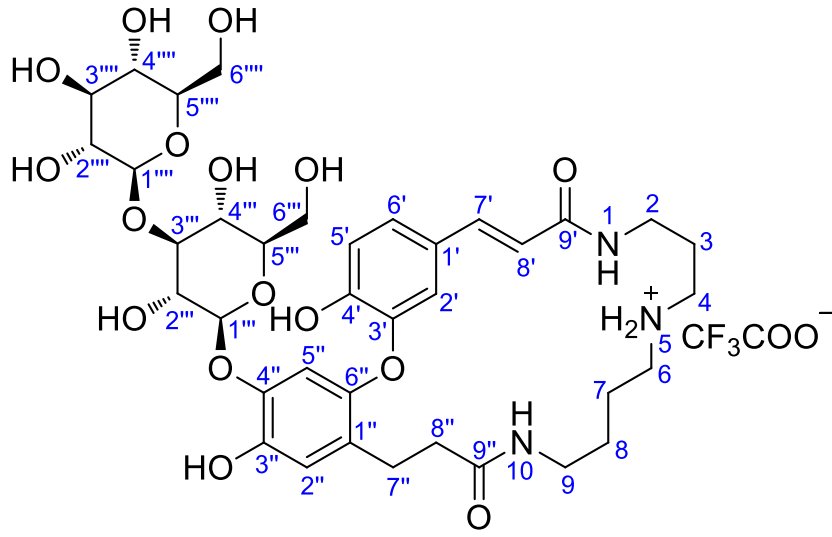

15

Table S15. NMR (600 MHz for ${ }^{1} \mathrm{H}$ NMR, $150 \mathrm{MHz}$ for ${ }^{13} \mathrm{C}$ NMR, DMSO- $d_{6}$ ) assignments of 15.

\begin{tabular}{|c|c|c|c|c|c|}
\hline No. & $\delta_{\mathrm{C}}$, type & $\delta_{\mathrm{H}}(J$ in $\mathrm{Hz})$ & ${ }^{1} \mathrm{H}-{ }^{1} \mathrm{H}$ COSY & HMBC & NOESY \\
\hline 1 & & $8.16, \mathrm{t}(5.8)$ & 2 & $2,9^{\prime}$ & $3,4,8^{\prime}$ \\
\hline 2 & $35.2, \mathrm{CH}_{2}$ & $3.24, \mathrm{~m}$ & 1,3 & $3,4,9^{\prime}$ & \\
\hline 3 & $25.4, \mathrm{CH}_{2}$ & 1.73 , quint (6.4) & 2,4 & 2,4 & 1,5 \\
\hline 4 & $42.9, \mathrm{CH}_{2}$ & $2.83, \mathrm{~m}$ & 3,5 & 2,3 & 1 \\
\hline 5 & & 8.37, br s & 4,6 & & 3 \\
\hline 6 & $45.4, \mathrm{CH}_{2}$ & $2.86, \mathrm{~m}$ & 5,7 & $4,7,8$ & 8 \\
\hline 7 & $22.2, \mathrm{CH}_{2}$ & 1.51 , quint (6.8) & 6,8 & $6,8,9$ & 9 \\
\hline 8 & $25.8, \mathrm{CH}_{2}$ & 1.38 , quint (6.5) & 7,9 & $6,7,9$ & 6,10 \\
\hline 9 & $38.1, \mathrm{CH}_{2}$ & $3.03, \mathrm{~m}$ & 8,10 & $7,8,9^{\prime \prime}$ & 7 \\
\hline 10 & & $7.67, \mathrm{t}(5.3)$ & 9 & $9,9^{\prime \prime}$ & $8,8^{\prime \prime}$ \\
\hline $1^{\prime}$ & 126.2, C & & & & \\
\hline $2^{\prime}$ & $115.0, \mathrm{CH}$ & $6.83, \mathrm{~d}(2.0)$ & $6^{\prime}$ & $3^{\prime}, 4^{\prime}, 6^{\prime}, 7^{\prime}$ & $7^{\prime}, 8^{\prime}$ \\
\hline $3^{\prime}$ & 147.2, C & & & & \\
\hline $4^{\prime}$ & 149.0, C & & & & \\
\hline $5^{\prime}$ & $116.6, \mathrm{CH}$ & $6.90, \mathrm{~d}(8.2)$ & $6^{\prime}$ & $1^{\prime}, 2^{\prime}, 3^{\prime}, 4^{\prime}$ & 4'-OH \\
\hline $6^{\prime}$ & $123.6, \mathrm{CH}$ & $7.08, \mathrm{dd}(8.3,1.9)$ & $2^{\prime}, 5^{\prime}$ & $2^{\prime}, 3^{\prime}, 4^{\prime}, 5^{\prime}, 7^{\prime}$ & $7^{\prime}, 8^{\prime}$ \\
\hline $7^{\prime}$ & $139.2, \mathrm{CH}$ & $7.26, \mathrm{~d}(15.7)$ & $8^{\prime}$ & $1^{\prime}, 2^{\prime}, 6^{\prime}, 8^{\prime}, 9^{\prime}$ & $2^{\prime}, 6^{\prime}$ \\
\hline $8^{\prime}$ & $118.6, \mathrm{CH}$ & $6.21, \mathrm{~d}(15.7)$ & $7^{\prime}$ & $1^{\prime}, 7^{\prime}, 9^{\prime}$ & $1,2^{\prime}, 6^{\prime}$ \\
\hline $9^{\prime}$ & 166.2, C & & & & \\
\hline $1 "$ & 127.8, C & & & & \\
\hline $2^{\prime \prime}$ & $116.9, \mathrm{CH}$ & $6.83, \mathrm{~s}$ & & 1", 3", 4", 5", 6", 7" & 3"-OH, 7", 8" \\
\hline 3" & $143.7, \mathrm{C}$ & & & & \\
\hline $4^{\prime \prime}$ & 143.8, C & & & & \\
\hline $5^{\prime \prime}$ & 109.9, CH & $6.68, \mathrm{~s}$ & & 1", 3", 4", 6", 7" & 1"', 2"', 5"' \\
\hline $6^{\prime \prime}$ & 145.1, C & & & & \\
\hline 7" & $23.3, \mathrm{CH}_{2}$ & $2.70, \mathrm{t}(7.2)$ & $8^{\prime \prime}$ & 1", 2", 6", 8", 9" & $2^{\prime \prime}$ \\
\hline $8^{\prime \prime}$ & $35.1, \mathrm{CH}_{2}$ & $2.33, \mathrm{t}(7.3)$ & $7 "$ & 1", 7", 9" & $10,2^{\prime \prime}$ \\
\hline
\end{tabular}




\begin{tabular}{|c|c|c|c|c|c|}
\hline $9^{\prime \prime}$ & 171.1, C & & & & \\
\hline $1 " '$ & $101.3, \mathrm{CH}$ & $4.77, \mathrm{~d}(7.3)$ & $2 " '$ & 4", 3"', 5"' & 5", 3'", 5"' \\
\hline $2^{\prime \prime \prime}$ & $72.1, \mathrm{CH}$ & $3.46, \mathrm{~m}$ & 1"', 3"', 2"'-OH & & $5^{\prime \prime}$ \\
\hline 3"' & $86.3, \mathrm{CH}$ & $3.46, \mathrm{~m}$ & 2"', 4"' & & 1"', $1 " ' "$ \\
\hline $4 " '$ & $67.7, \mathrm{CH}$ & $3.31, \mathrm{~m}$ & 3"', 5"', 4"'-OH & 3'", 5"' & \\
\hline $5^{\prime \prime \prime}$ & $76.3, \mathrm{CH}$ & $3.27, \mathrm{~m}$ & 4"', 6"'-Ha, 6"'-Hb & 3'", 4"', 6"' & 5", 1"' \\
\hline \multirow[t]{2}{*}{ 6"' } & $60.1, \mathrm{CH}_{2}$ & 3.54, br d (11.0), Ha & 5"', 6"'-Hb & & \\
\hline & & $3.46, \mathrm{~m}, \mathrm{Hb}$ & 5"', 6"'-На & & \\
\hline $1^{\prime \prime \prime \prime}$ & $103.8, \mathrm{CH}$ & $4.36, \mathrm{~d}(7.8)$ & $2 " '$ & 3"', $2^{\prime \prime \prime \prime}, 3^{\prime \prime \prime}, 5^{\prime \prime \prime \prime}$ & 3"', 3"'", 5"'" \\
\hline $2^{\prime \prime \prime \prime}$ & $73.8, \mathrm{CH}$ & $3.06, \mathrm{~m}$ & 1"'", 3 "'"' & & \\
\hline $3^{\prime \prime \prime \prime}$ & $76.1, \mathrm{CH}$ & $3.16, \mathrm{~m}$ & 2"'", 4"'"' & & $1 " '$ \\
\hline 4"'" & $70.1, \mathrm{CH}$ & $3.04, \mathrm{~m}$ & 3"', 5 "'"' & & \\
\hline $5^{\prime \prime \prime \prime}$ & $76.9, \mathrm{CH}$ & $3.16, \mathrm{~m}$ & 4"', 6"''-Ha, 6"'--Hb & & $1^{\prime \prime \prime}$ \\
\hline \multirow[t]{2}{*}{ 6"' } & $61.1, \mathrm{CH}_{2}$ & 3.68, br d (10.9), Нa & $5^{\prime \prime "}, 6$ "''-Hb & & \\
\hline & & $3.39, \mathrm{~m}, \mathrm{Hb}$ & 5"', 6 "''-На & & \\
\hline 4'-OH & & $9.88, \mathrm{~s}$ & & $3^{\prime}, 4^{\prime}, 5^{\prime}$ & $5^{\prime}$ \\
\hline 3"-OH & & $8.55, \mathrm{~s}$ & & 1", 2", 3", 4" & 2", 2"'-OH \\
\hline 2"'-OH & & $5.61, \mathrm{~d}(3.4)$ & $2 " '$ & 1"', 2"', 3"' & 3"--OH \\
\hline 4"'-OH & & 4.67, d (2.5) & 4"' & 3"', 4"', 5"' & \\
\hline
\end{tabular}




\section{The result of short-term memory assay on the transgenic fly AD model of 1-15}

Table S16. Performance indexes (PI) of AD flies fed with 1-15.

\begin{tabular}{|c|c|c|c|c|c|}
\hline \multicolumn{2}{|c|}{$\mathrm{a}$} & \multicolumn{2}{|c|}{$\mathrm{b}$} & \multicolumn{2}{|c|}{$\mathrm{c}$} \\
\hline \multicolumn{2}{|c|}{ Genotype/drug PI $(100 \mu \mathrm{M})$} & \multicolumn{2}{|c|}{ Genotype/drug PI $(100 \mu \mathrm{M})$} & \multicolumn{2}{|c|}{ Genotype/drug PI $(100 \mu \mathrm{M})$} \\
\hline $2 \mathrm{U}$ & $71.2 \pm 1.4$ & $2 \mathrm{U}$ & $72.7 \pm 1.2$ & $2 \mathrm{U}$ & $68.9 \pm 2.0$ \\
\hline $2 \mathrm{U}^{*} \mathrm{H} 29.3$ & $51.0 \pm 1.3$ & $2 \mathrm{U} * \mathrm{H} 29.3$ & $50.1 \pm 1.0$ & $2 \mathrm{U}^{*} \mathrm{H} 29.3$ & $50.2 \pm 1.2$ \\
\hline $\mathrm{P} 35 * \mathrm{H} 29.3$ & $27.2 \pm 1.6$ & $\mathrm{P} 35^{*} \mathrm{H} 29.3$ & $22.4 \pm 2.7$ & $\mathrm{P} 35^{*} \mathrm{H} 29.3$ & $23.3 \pm 3.4$ \\
\hline $\mathrm{PC}$ & $43.1 \pm 0.9$ & $\mathrm{PC}$ & $42.7 \pm 0.9$ & $\mathrm{PC}$ & $44.2 \pm 2.1$ \\
\hline 1 & $35.5 \pm 3.8$ & 6 & $44.6 \pm 2.5$ & 13 & $44.6 \pm 1.2$ \\
\hline 2 & $42.9 \pm 1.6$ & 7 & $32.2 \pm 2.3$ & 14 & $37.1 \pm 2.2$ \\
\hline 3 & $42.2 \pm 2.1$ & $8 \& 9$ & $39.8 \pm 2.4$ & 15 & $45.9 \pm 1.9$ \\
\hline 4 & $35.0 \pm 1.3$ & 10 & $37.2 \pm 2.9$ & & \\
\hline \multirow[t]{2}{*}{5} & $40.2 \pm 3.1$ & 11 & $33.5 \pm 3.0$ & & \\
\hline & & 12 & $42.5 \pm 3.0$ & & \\
\hline
\end{tabular}

2U: wild type flies; $2 \mathrm{U}^{*} \mathrm{H} 29.3$ : normal flies; P35*H29.3: AD flies without drug treatment; PC: AD flies with memantine treatment; PI: performance index $(100=$ perfect learning; $0=$ no learning $)$. 


\section{The 1D and 2D NMR spectra of 1-15}

\section{The 1D and 2D NMR spectra of 1.}

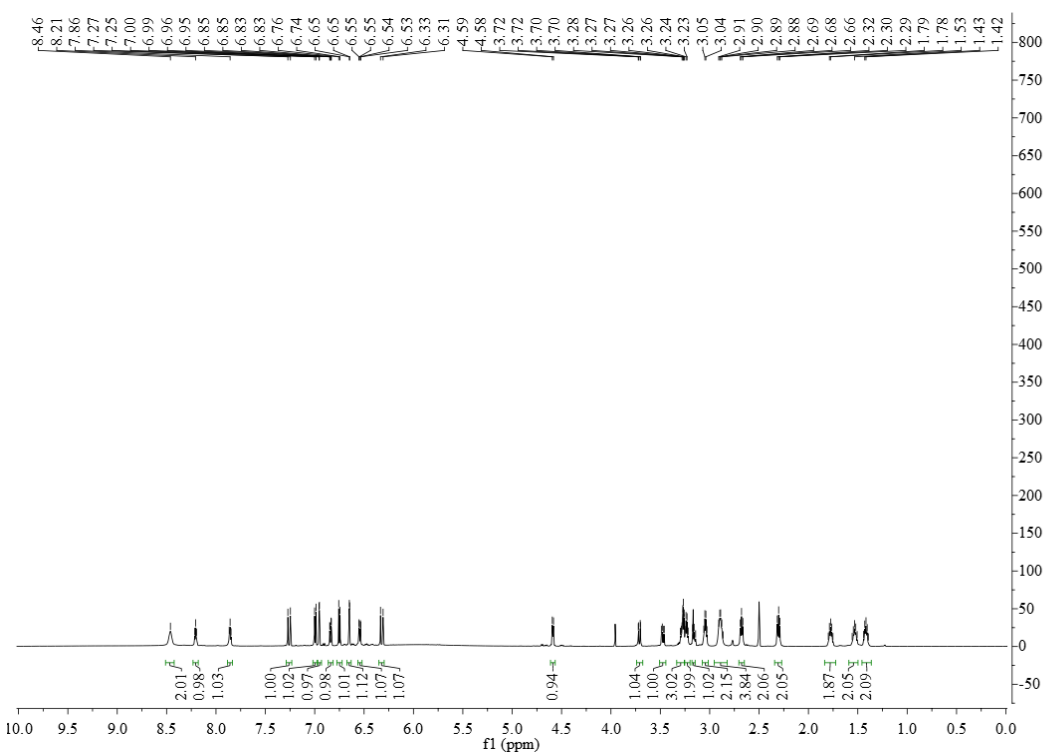

Figure S1. ${ }^{1} \mathrm{H}$ NMR spectrum of lycibarbarspermidine A (1) in DMSO- $d_{6}$ at $600 \mathrm{MHz}$

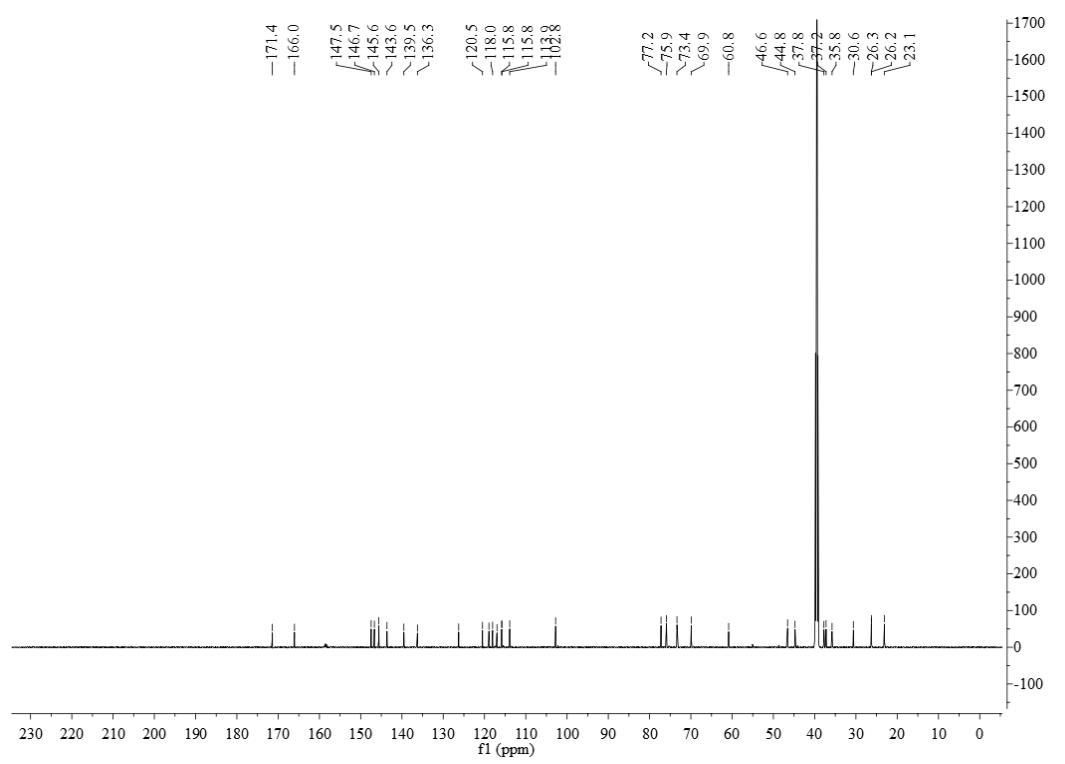

Figure S2. ${ }^{13} \mathrm{C}$ NMR spectrum of lycibarbarspermidine A (1) in DMSO- $d_{6}$ at $150 \mathrm{MHz}$ 


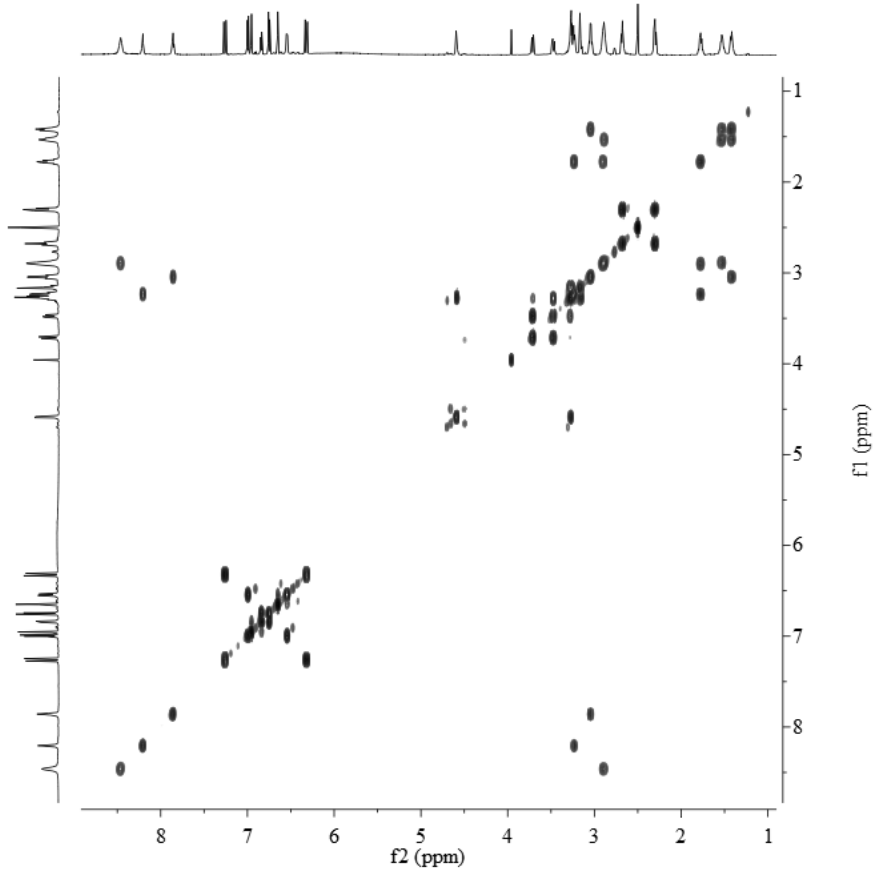

Figure S3. ${ }^{1} \mathrm{H}-{ }^{1} \mathrm{H}$ COSY spectrum of lycibarbarspermidine A (1) in DMSO- $d_{6}$ at $600 \mathrm{MHz}$

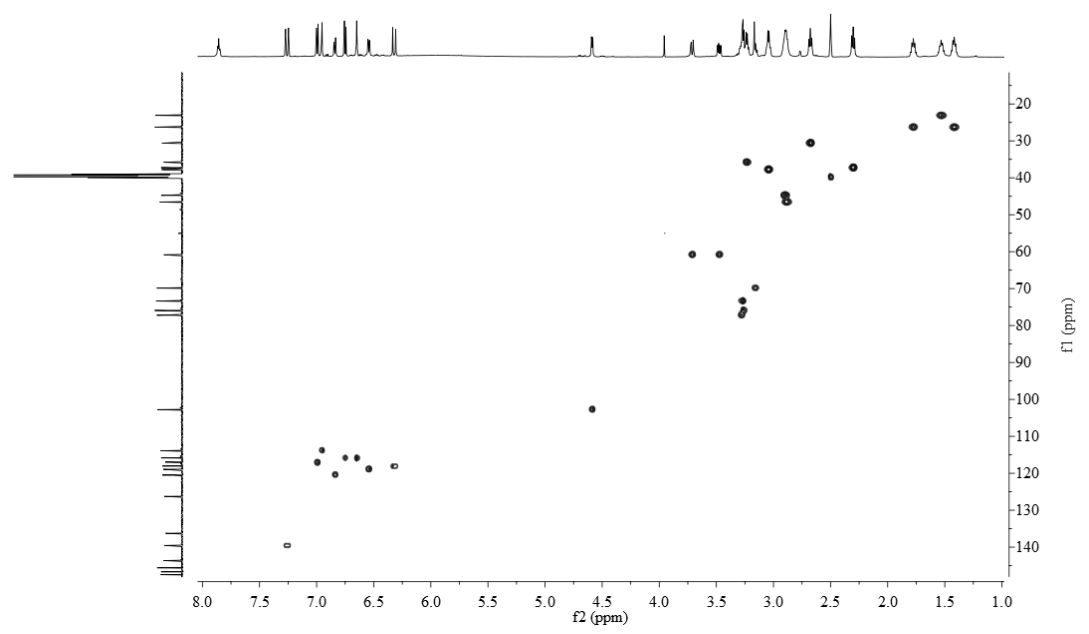

Figure S4. HSQC spectrum of lycibarbarspermidine A (1) in DMSO- $d_{6}$ at $600 \mathrm{MHz}$ 


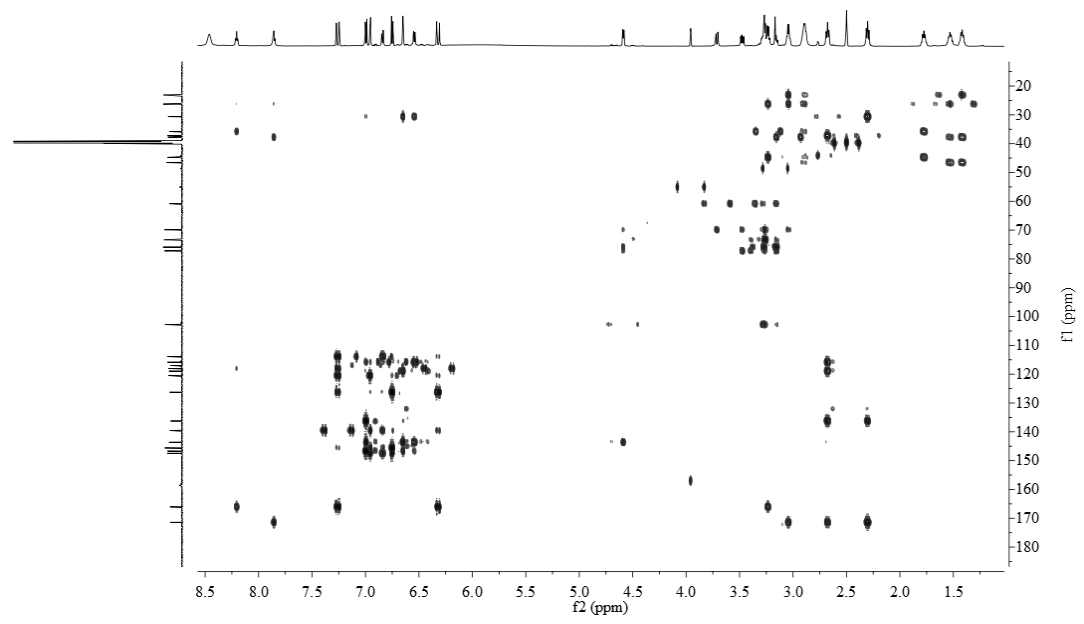

Figure S5. HMBC spectrum of lycibarbarspermidine A (1) in DMSO- $d_{6}$ at $600 \mathrm{MHz}$

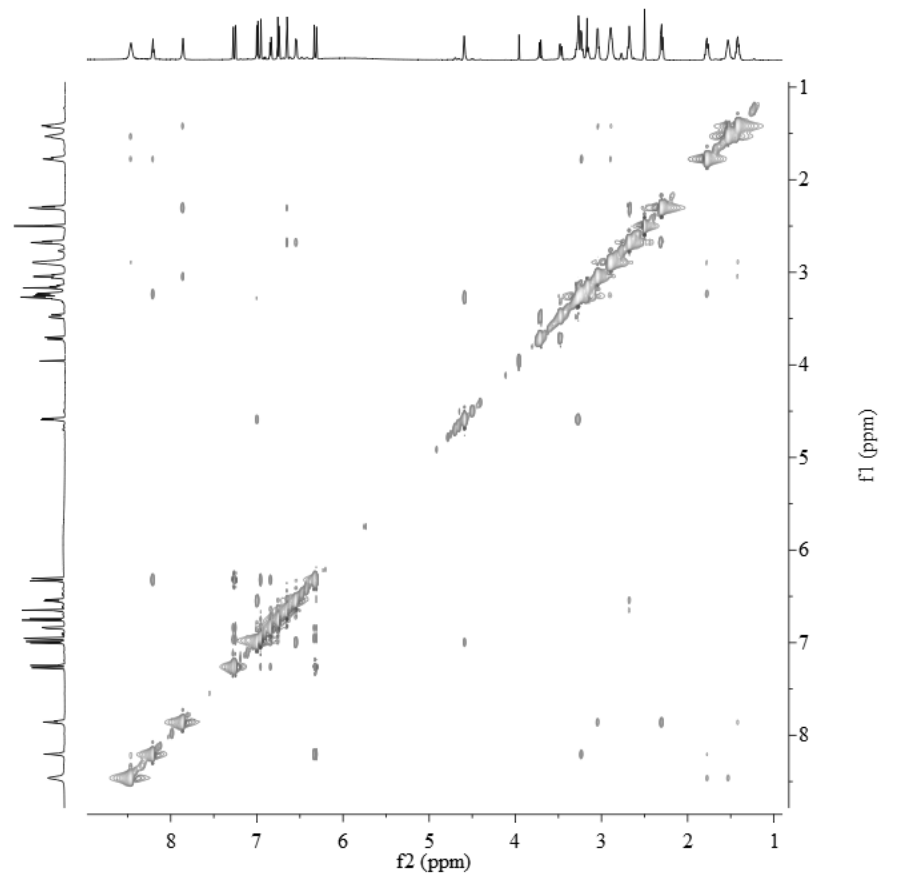

Figure S6. NOESY spectrum of lycibarbarspermidine A (1) in DMSO- $d_{6}$ at $600 \mathrm{MHz}$ 


\section{The 1D and 2D NMR spectra of 2.}

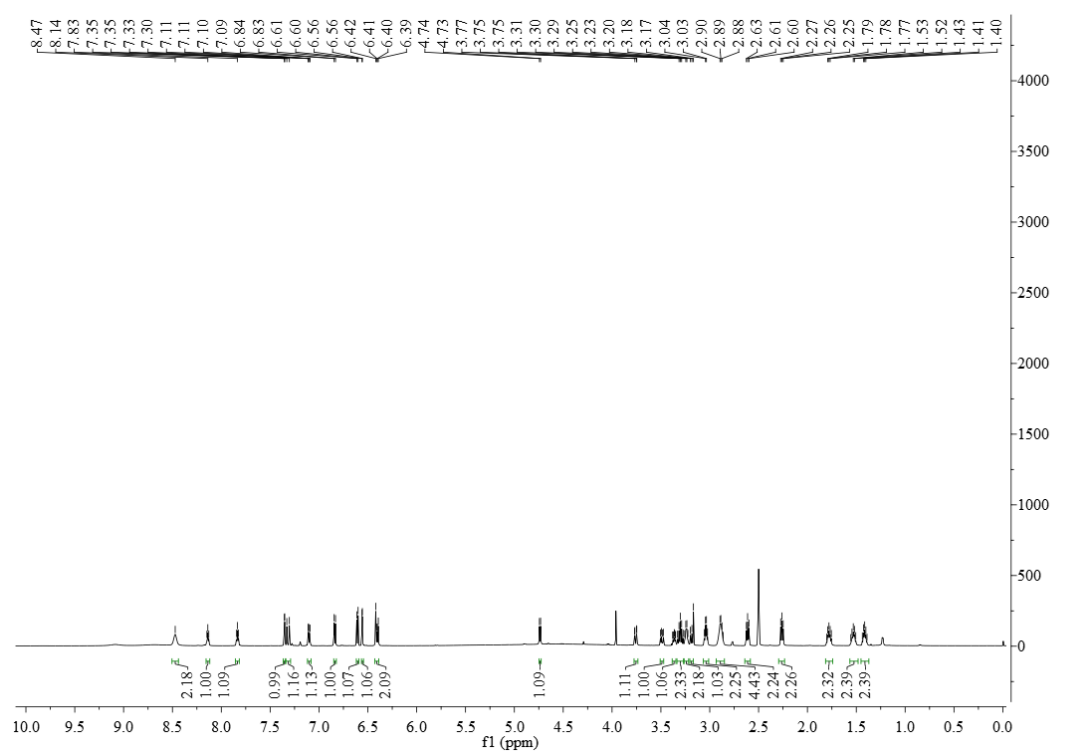

Figure S7. ${ }^{1} \mathrm{H}$ NMR spectrum of lycibarbarspermidine B (2) in DMSO- $d_{6}$ at $600 \mathrm{MHz}$

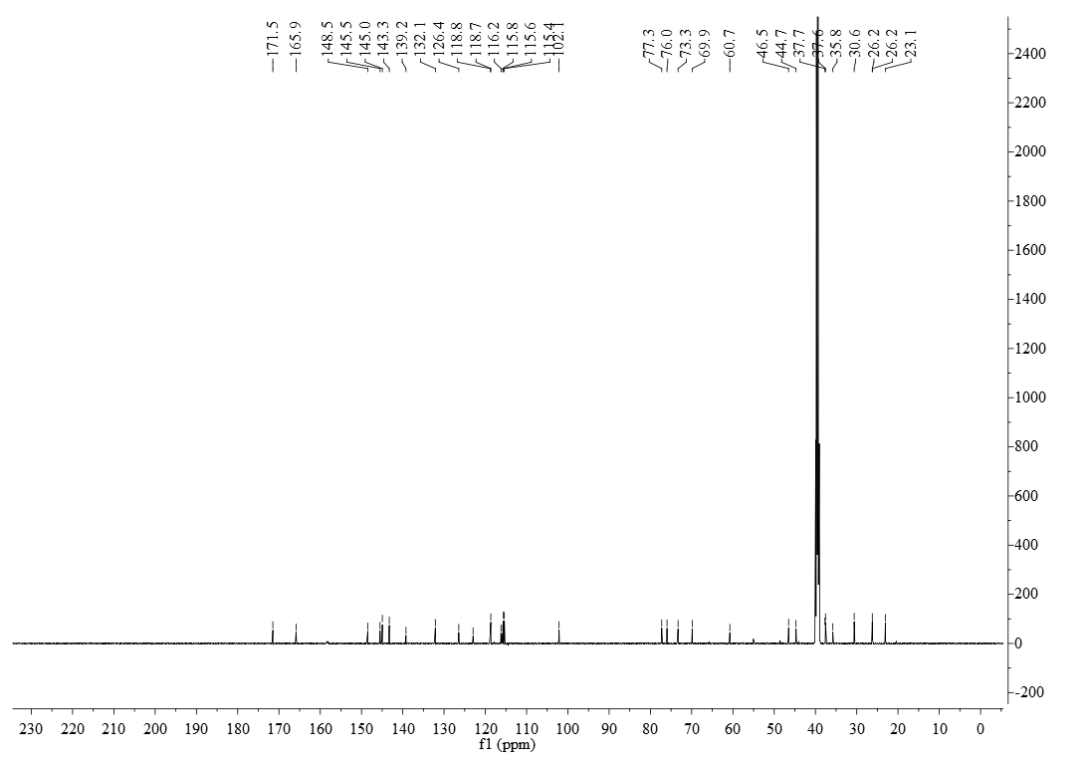

Figure S8. ${ }^{13} \mathrm{C}$ NMR spectrum of lycibarbarspermidine B (2) in DMSO- $d_{6}$ at $150 \mathrm{MHz}$ 


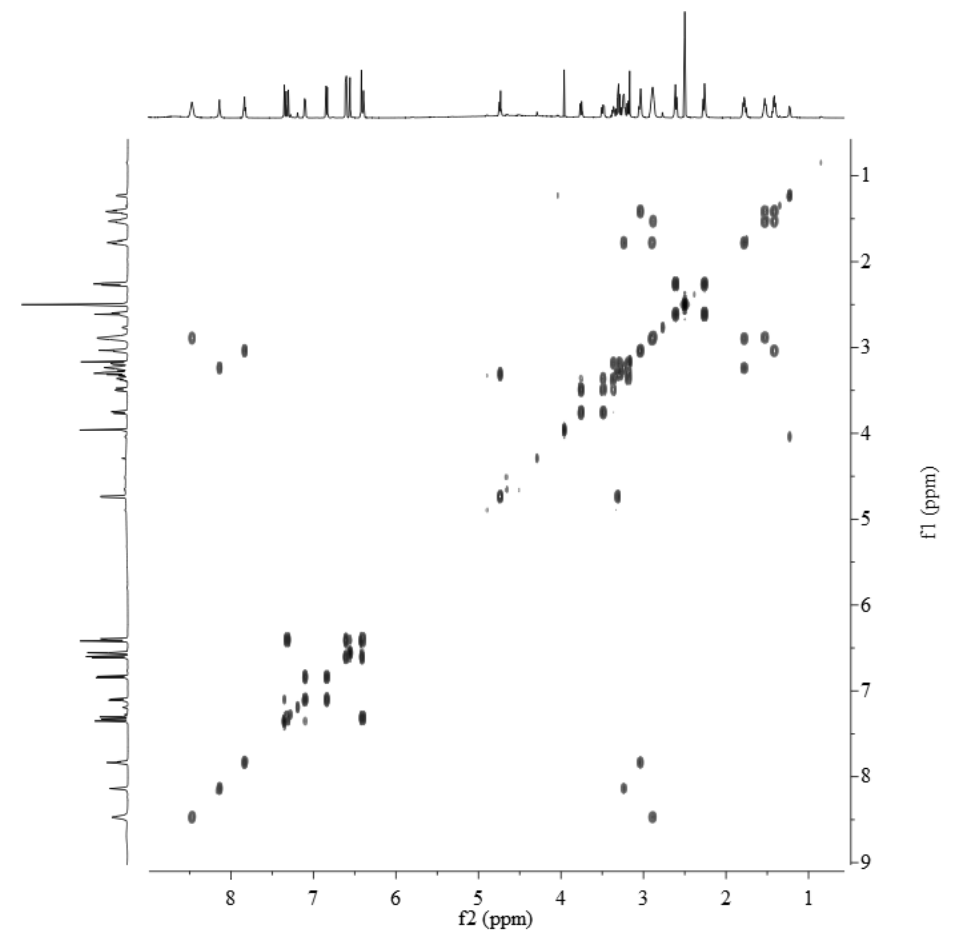

Figure S9. ${ }^{1} \mathrm{H}-{ }^{1} \mathrm{H}$ COSY spectrum of lycibarbarspermidine B (2) in DMSO- $d_{6}$ at $600 \mathrm{MHz}$

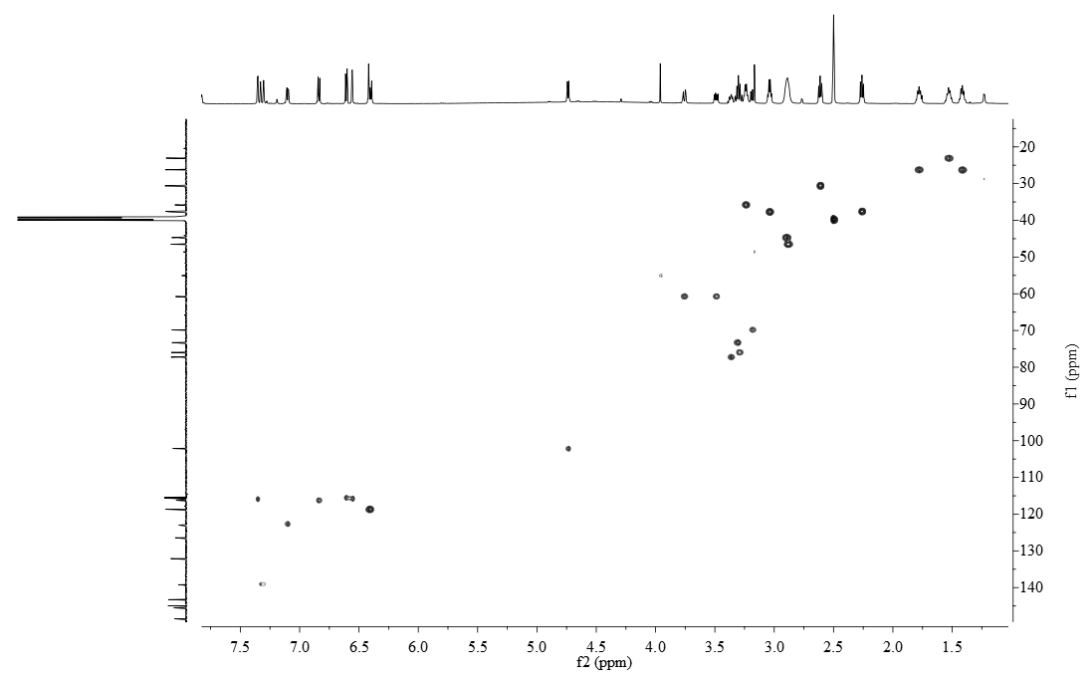

Figure S10. HSQC spectrum of lycibarbarspermidine B (2) in DMSO- $d_{6}$ at $600 \mathrm{MHz}$ 


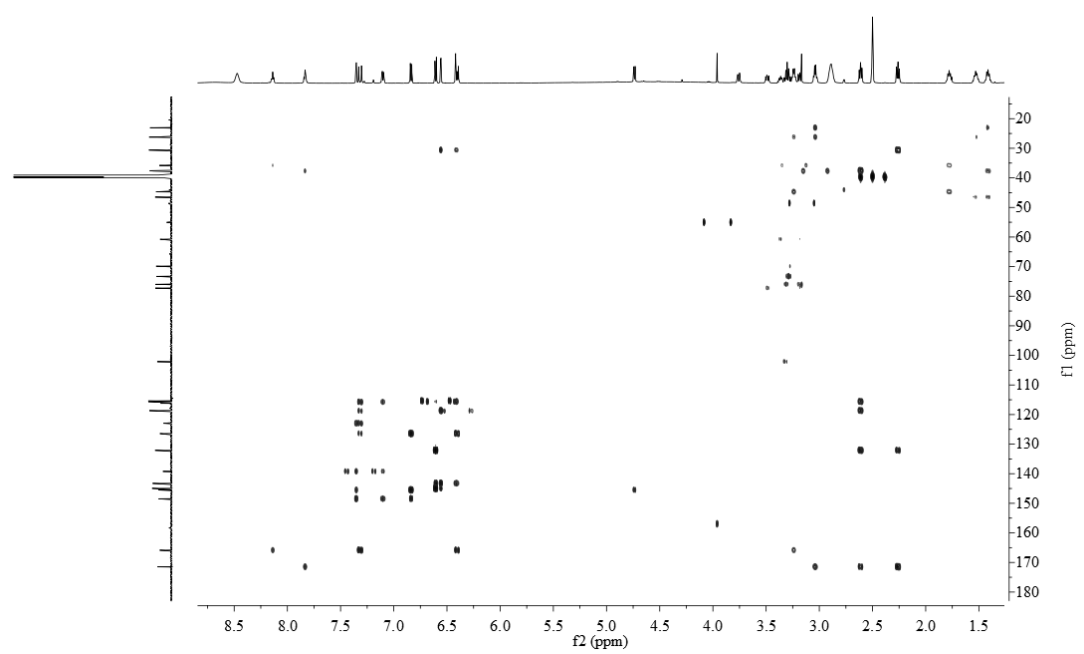

Figure S11. HMBC spectrum of lycibarbarspermidine B (2) in DMSO- $d_{6}$ at $600 \mathrm{MHz}$

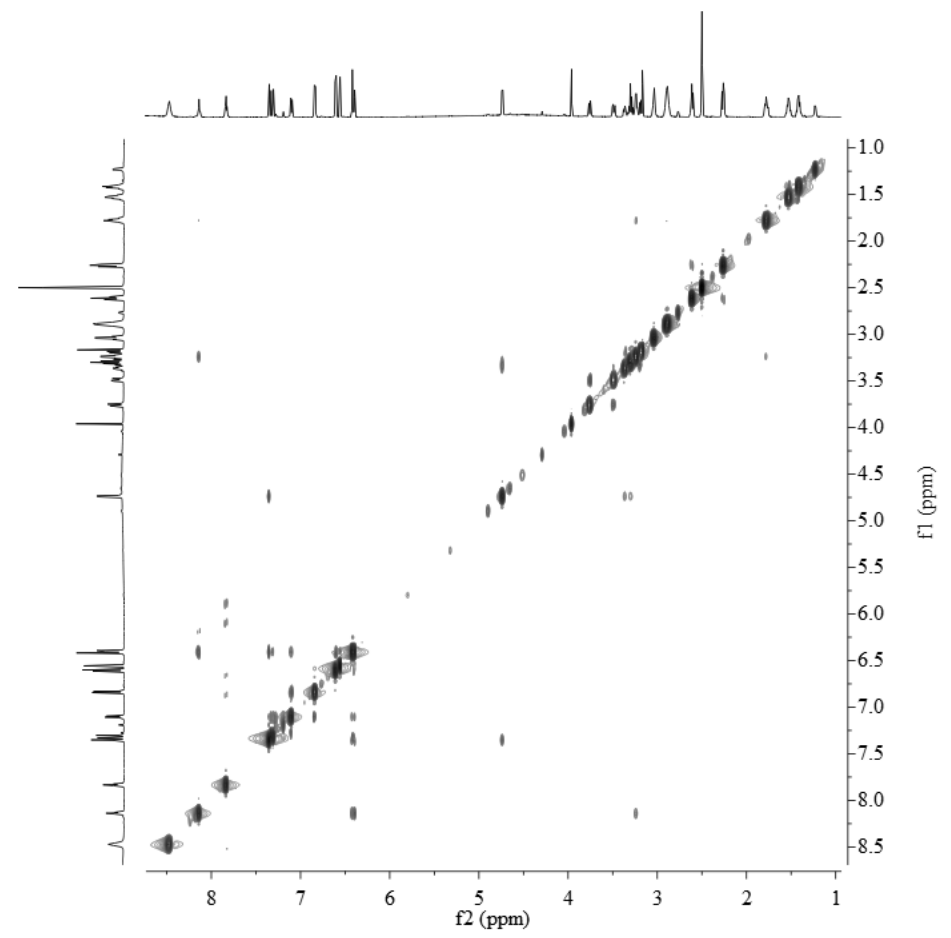

Figure S12. NOESY spectrum of lycibarbarspermidine B (2) in DMSO- $d_{6}$ at $600 \mathrm{MHz}$ 


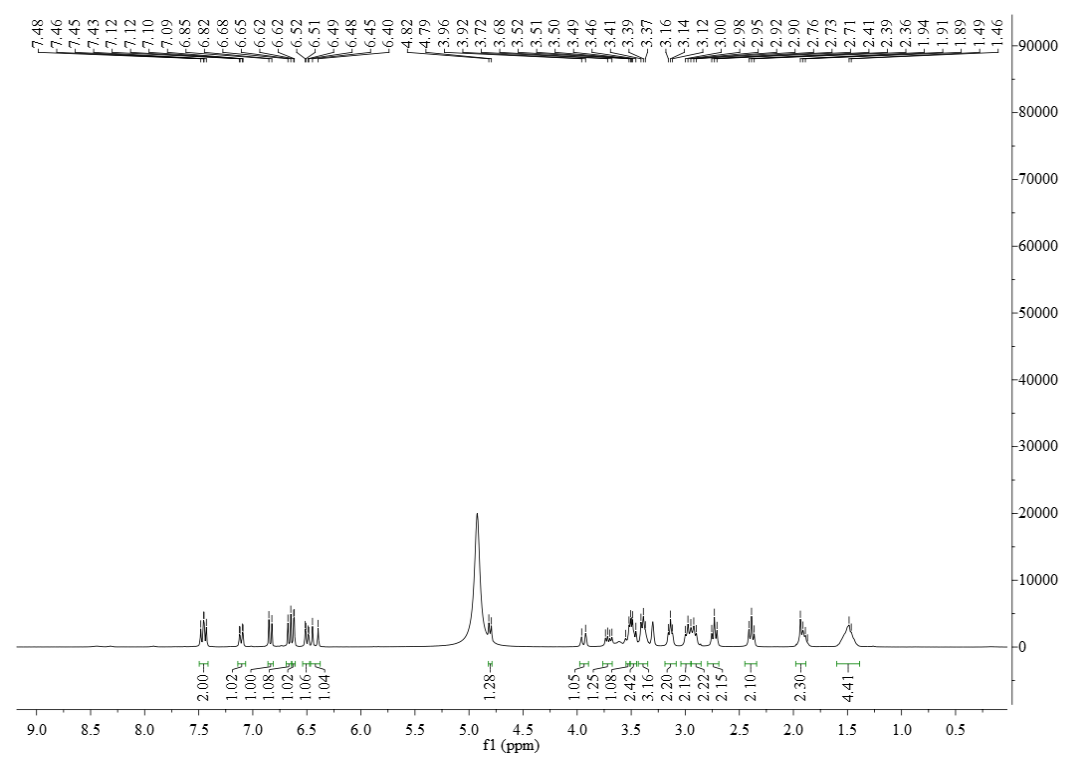

Figure S13. ${ }^{1} \mathrm{H}$ NMR spectrum of lycibarbarspermidine B (2) in $\mathrm{CD}_{3} \mathrm{OD}$ at $300 \mathrm{MHz}$

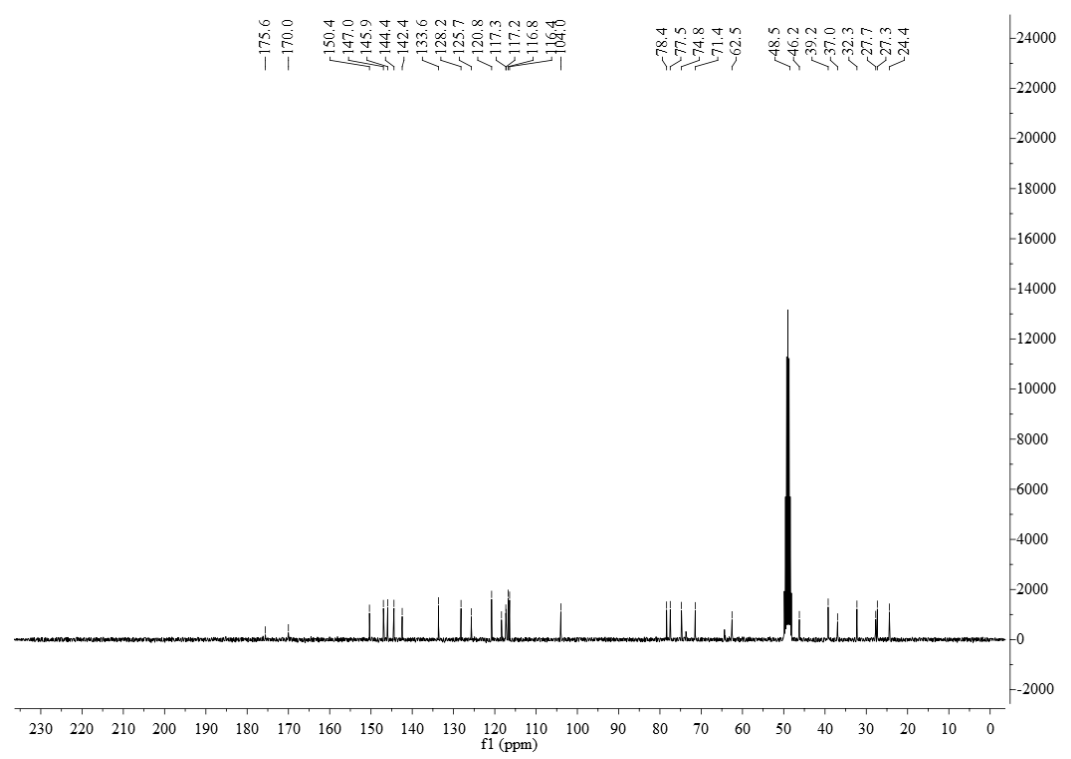

Figure S14. ${ }^{13} \mathrm{C}$ NMR spectrum of lycibarbarspermidine $\mathrm{B}(2)$ in $\mathrm{CD}_{3} \mathrm{OD}$ at $75 \mathrm{MHz}$ 


\section{The 1D and 2D NMR spectra of 3.}

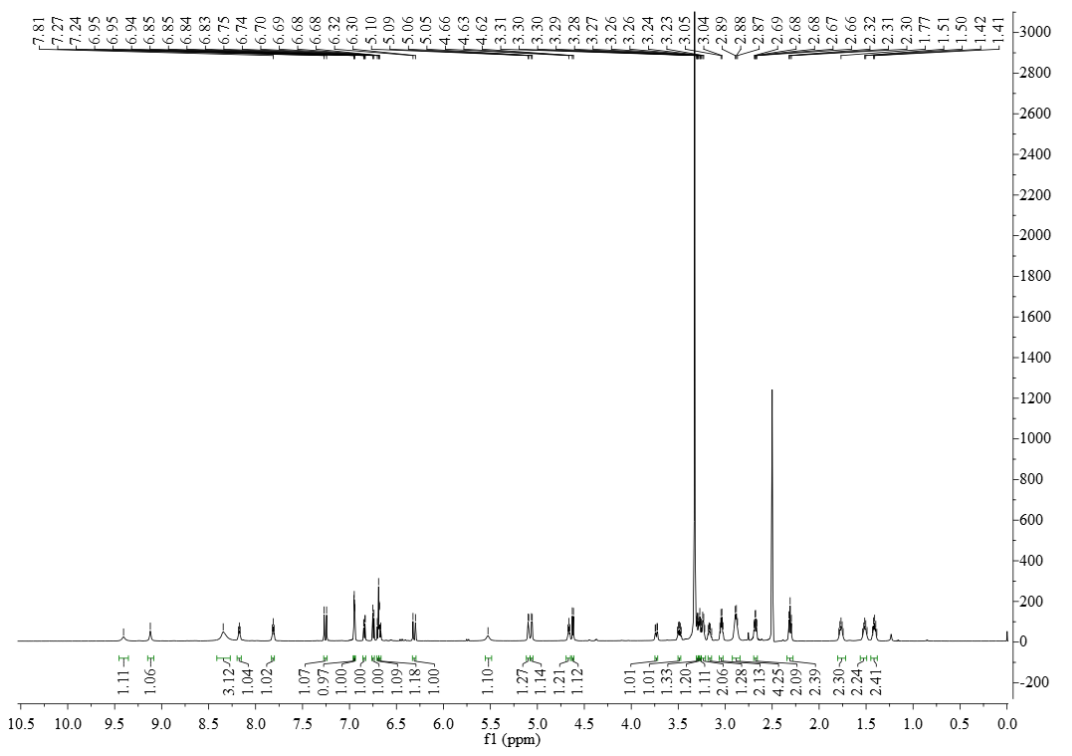

Figure S15. ${ }^{1} \mathrm{H}$ NMR spectrum of lycibarbarspermidine C (3) in DMSO- $d_{6}$ at $600 \mathrm{MHz}$

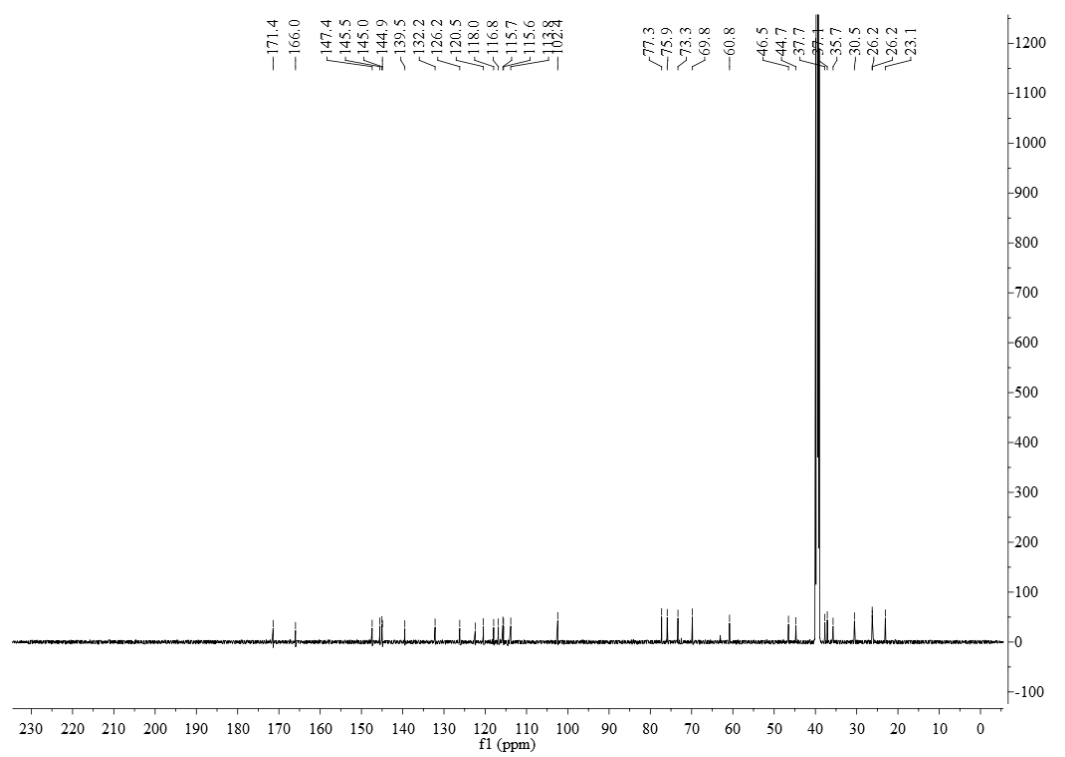

Figure S16. ${ }^{13} \mathrm{C}$ NMR spectrum of lycibarbarspermidine C (3) in DMSO- $d_{6}$ at $150 \mathrm{MHz}$ 


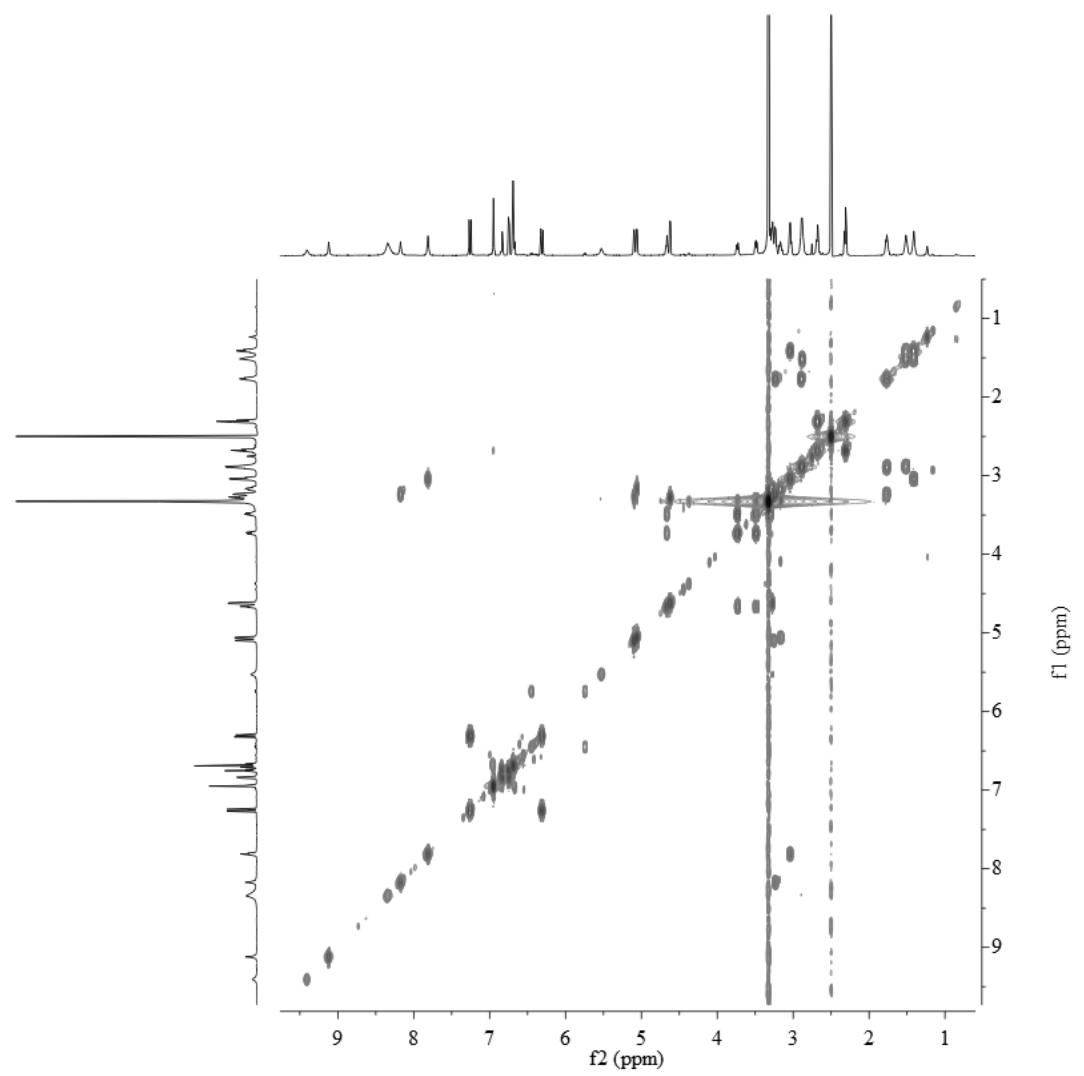

Figure S17. ${ }^{1} \mathrm{H}-{ }^{1} \mathrm{H}$ COSY spectrum of lycibarbarspermidine C (3) in DMSO- $d_{6}$ at $600 \mathrm{MHz}$

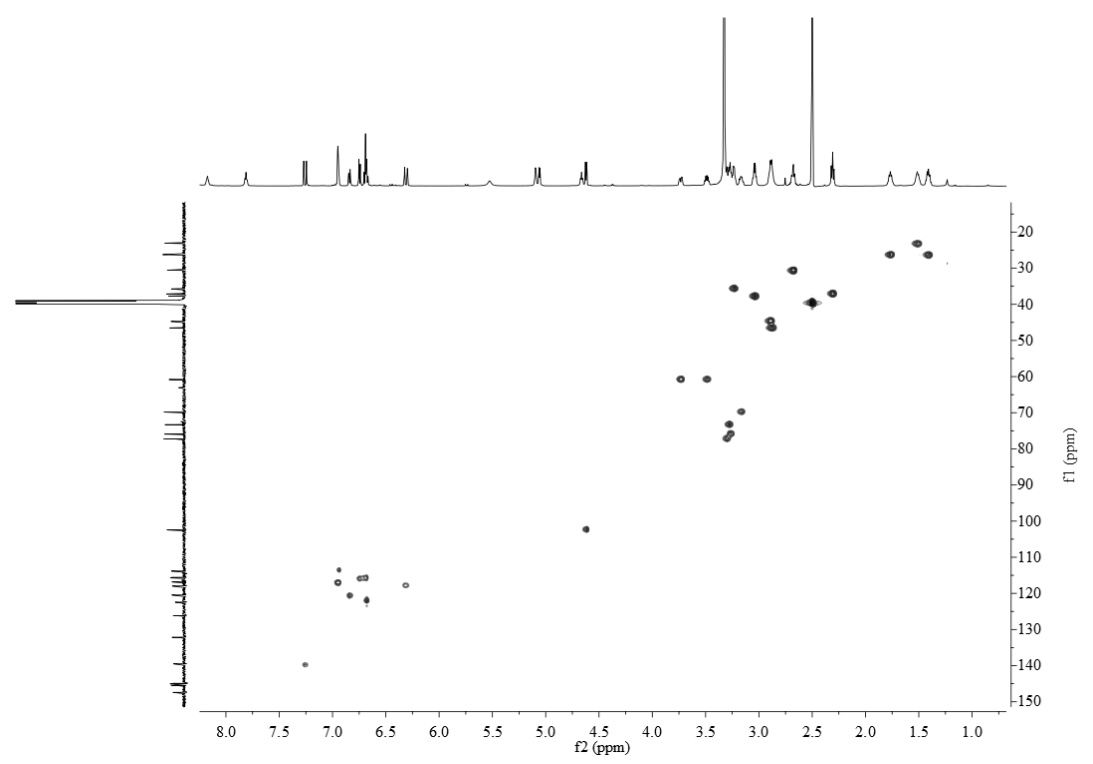

Figure S18. HSQC spectrum of lycibarbarspermidine C (3) in DMSO- $d_{6}$ at $600 \mathrm{MHz}$ 


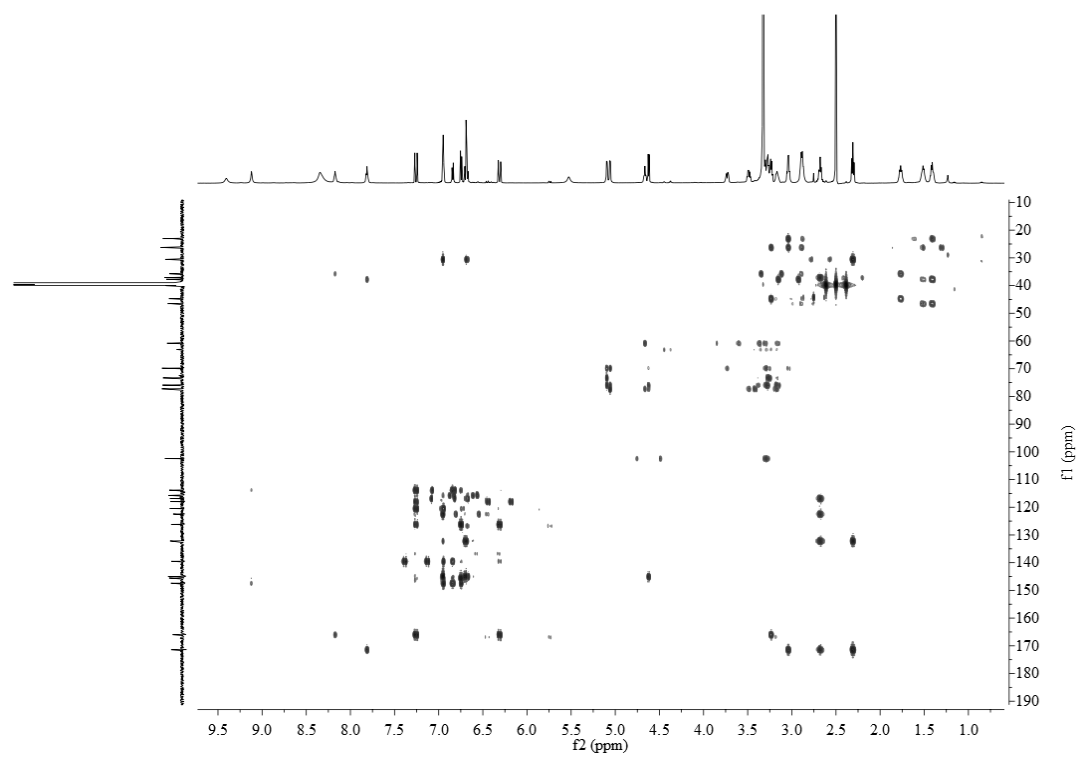

Figure S19. HMBC spectrum of lycibarbarspermidine C (3) in DMSO- $d_{6}$ at $600 \mathrm{MHz}$

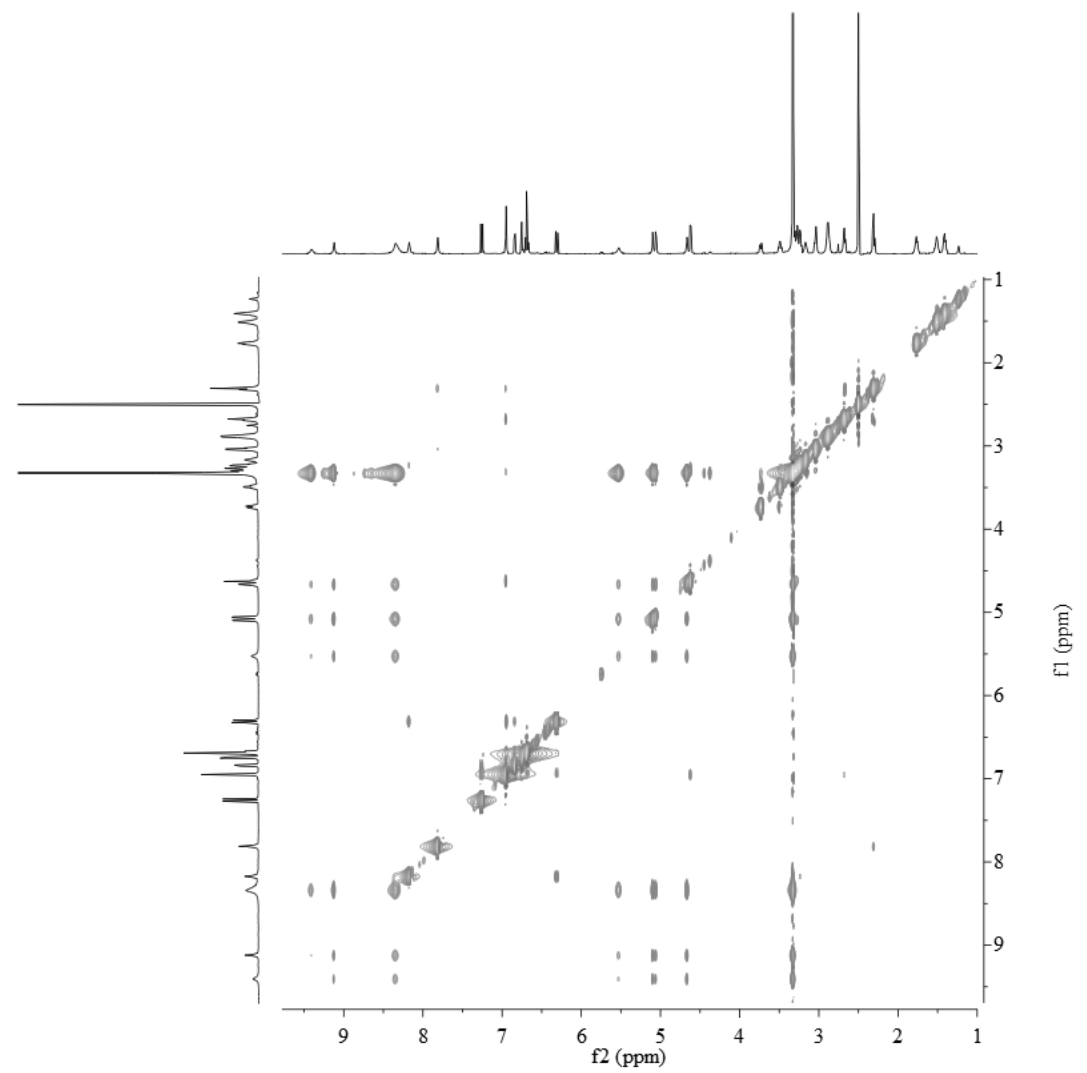

Figure S20. NOESY spectrum of lycibarbarspermidine C (3) in DMSO- $d_{6}$ at $600 \mathrm{MHz}$ 


\section{The 1D and 2D NMR spectra of 4.}

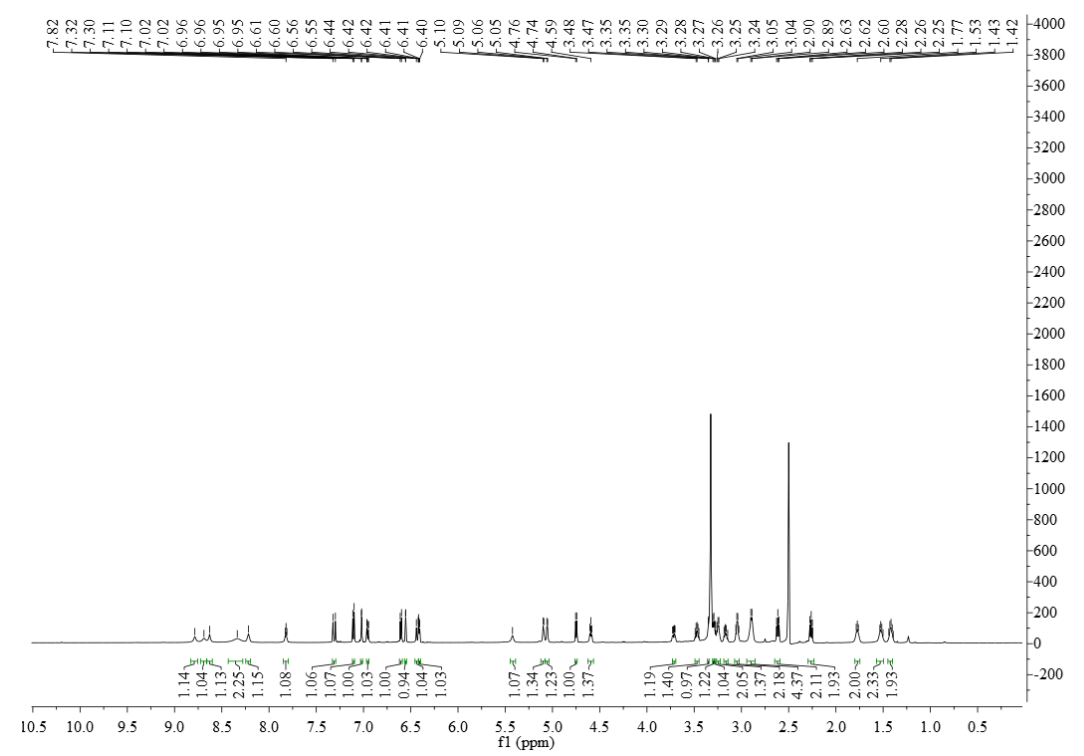

Figure S21. ${ }^{1} \mathrm{H}$ NMR spectrum of lycibarbarspermidine D (4) in DMSO- $d_{6}$ at $600 \mathrm{MHz}$

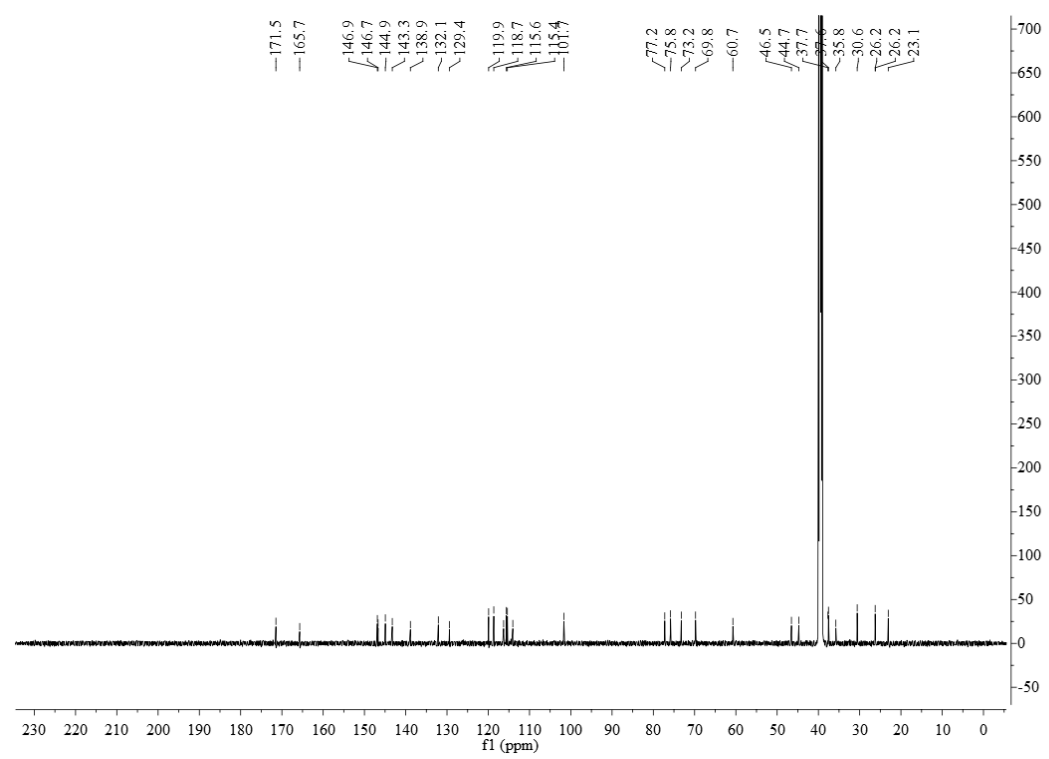

Figure S22. ${ }^{13} \mathrm{C}$ NMR spectrum of lycibarbarspermidine D (4) in DMSO- $d_{6}$ at $150 \mathrm{MHz}$ 


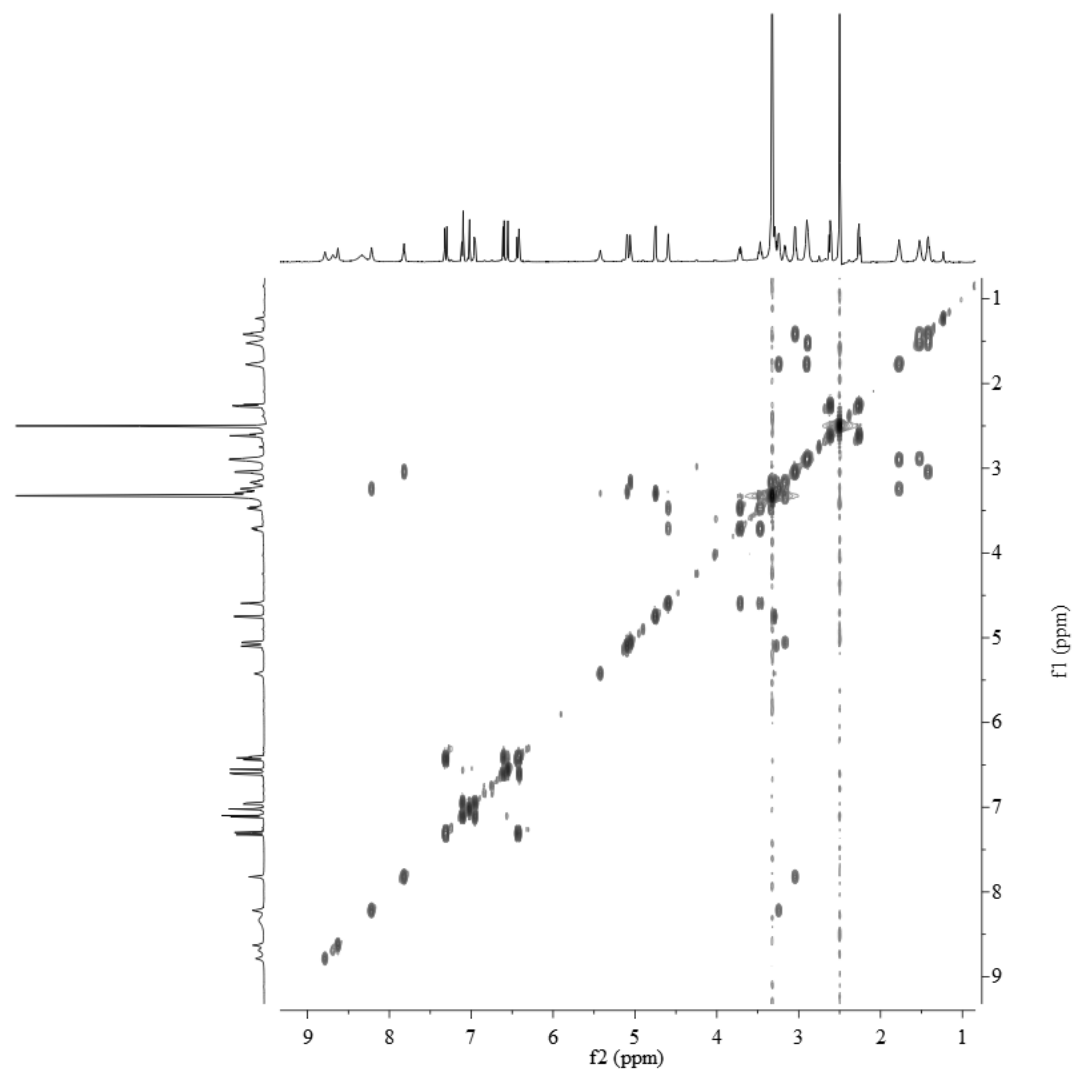

Figure S23. ${ }^{1} \mathrm{H}-{ }^{1} \mathrm{H}$ COSY spectrum of lycibarbarspermidine D (4) in DMSO- $d_{6}$ at $600 \mathrm{MHz}$

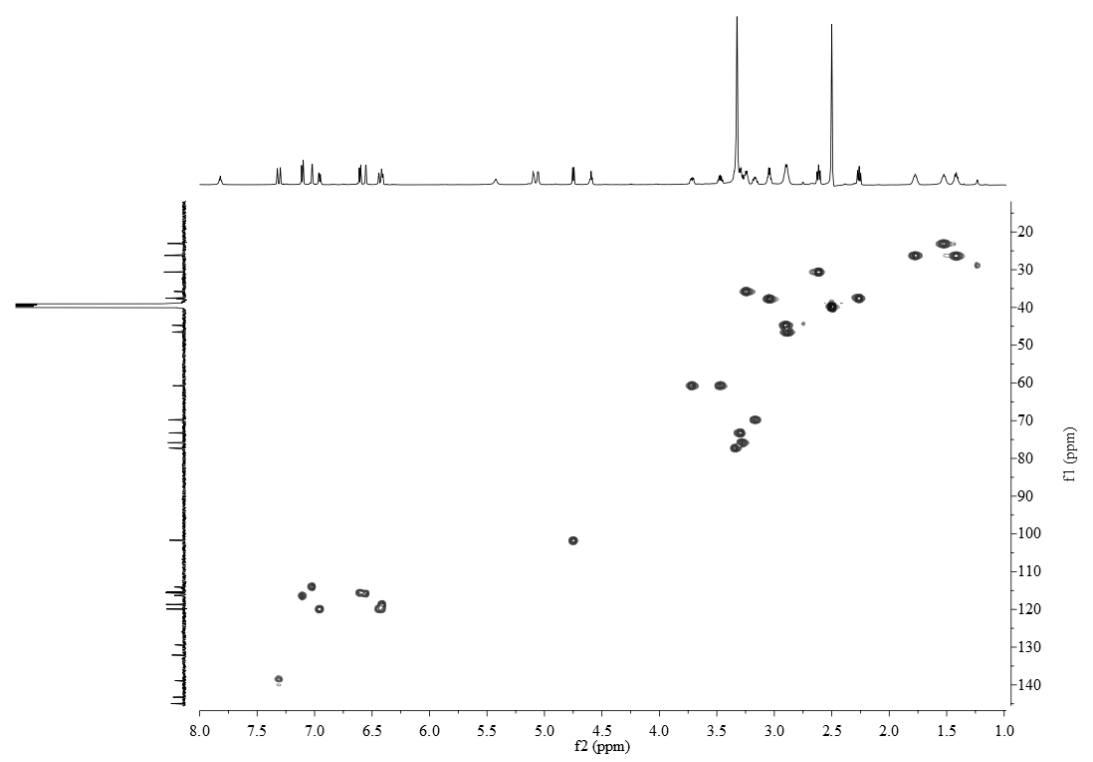

Figure S24. HSQC spectrum of lycibarbarspermidine D (4) in DMSO- $d_{6}$ at $600 \mathrm{MHz}$ 


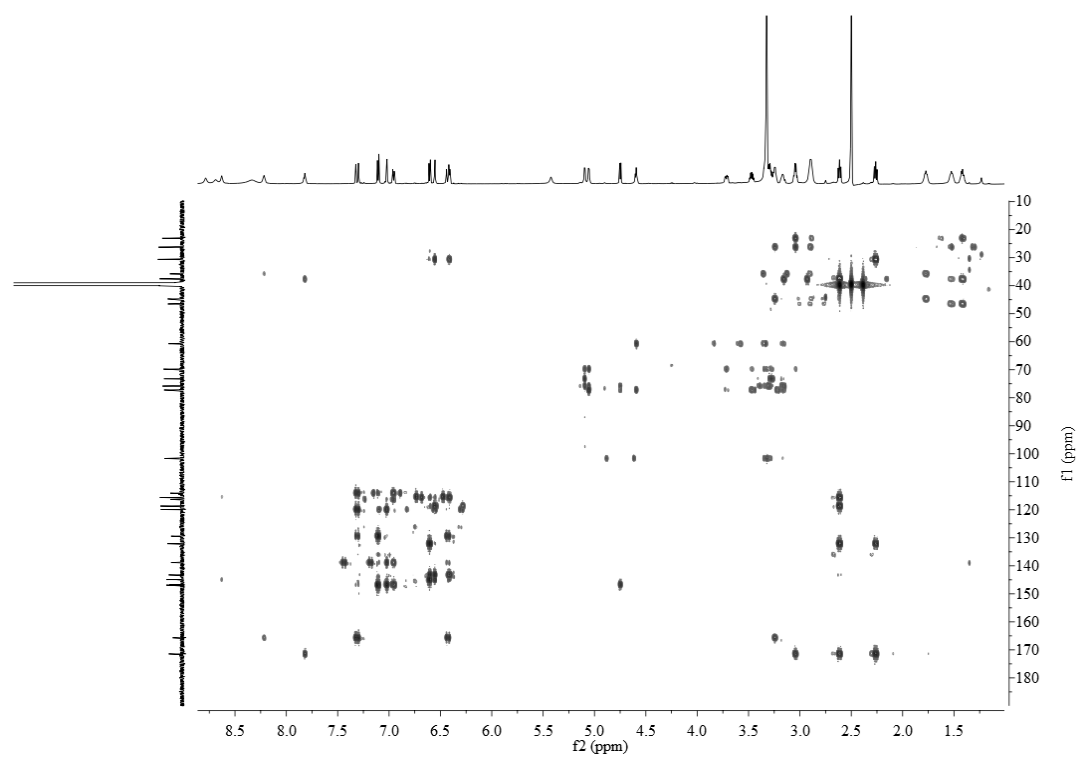

Figure S25. HMBC spectrum of lycibarbarspermidine D (4) in DMSO- $d_{6}$ at $600 \mathrm{MHz}$

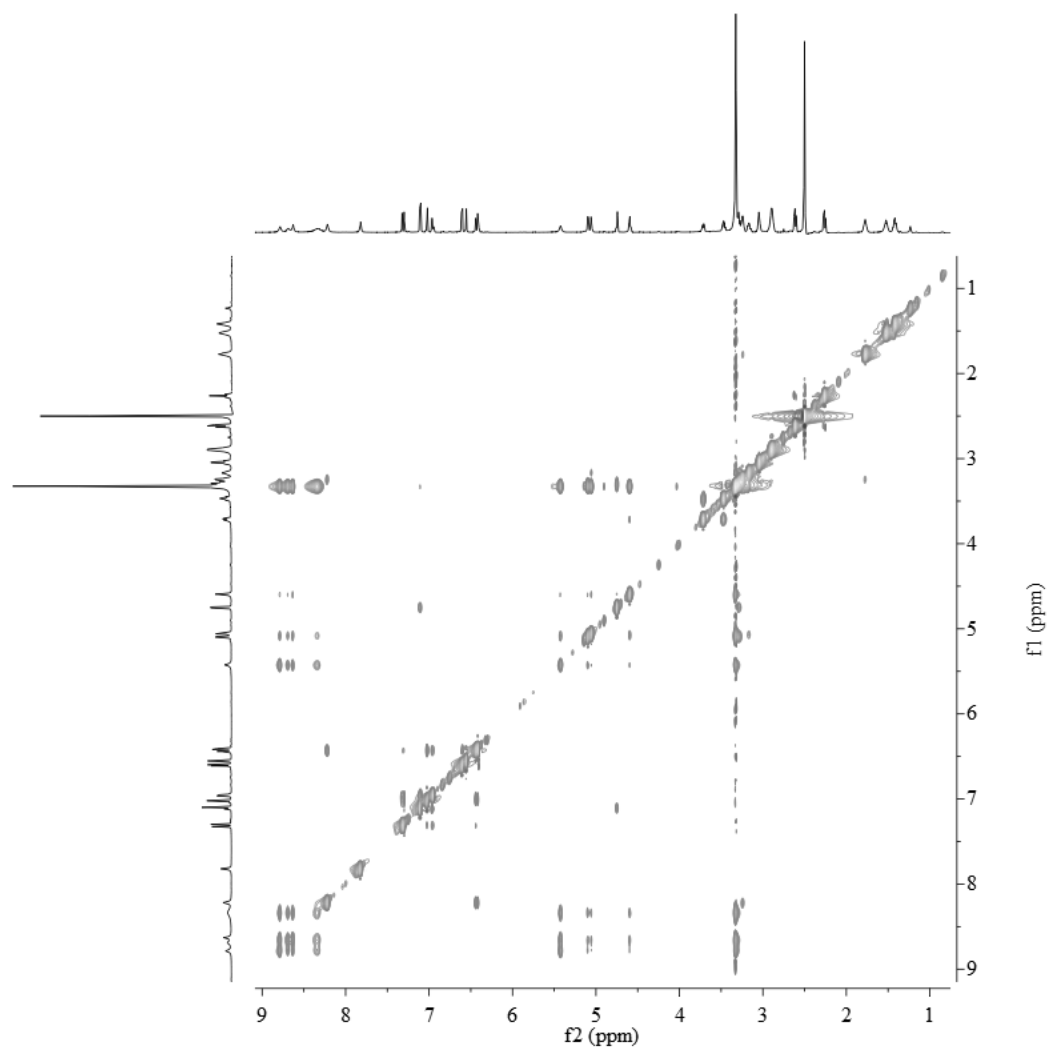

Figure S26. NOESY spectrum of lycibarbarspermidine D (4) in DMSO- $d_{6}$ at $600 \mathrm{MHz}$ 


\section{The 1D and 2D NMR spectra of 5.}

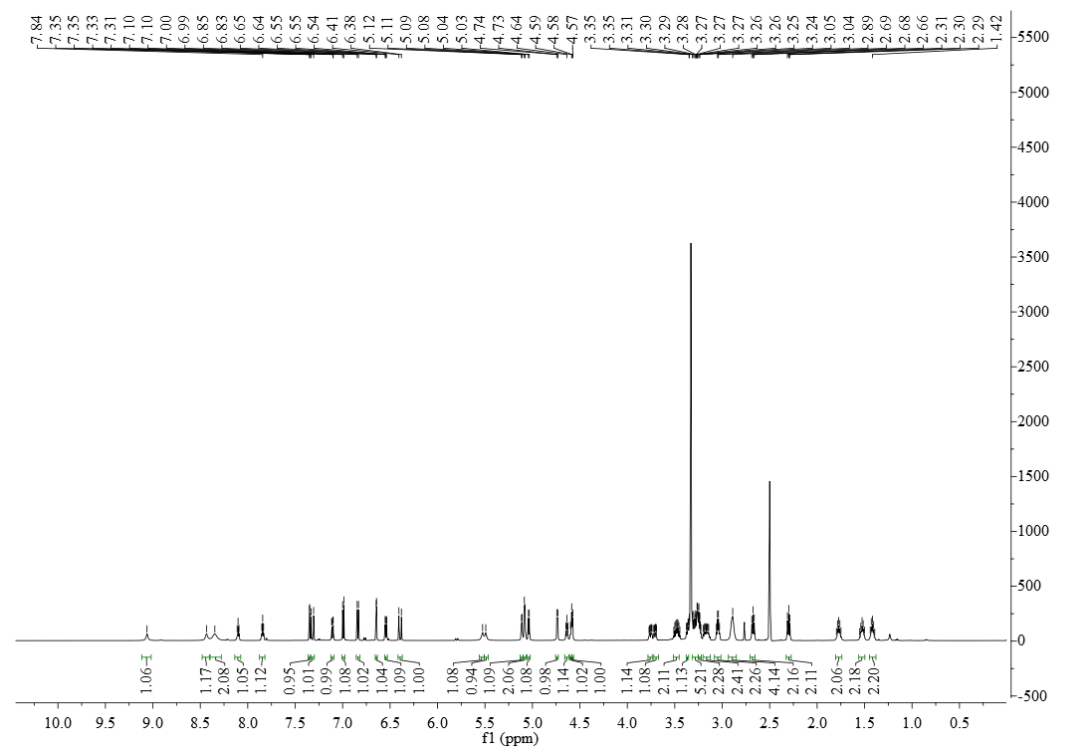

Figure S27. ${ }^{1} \mathrm{H}$ NMR spectrum of lycibarbarspermidine E (5) in DMSO- $d_{6}$ at $600 \mathrm{MHz}$

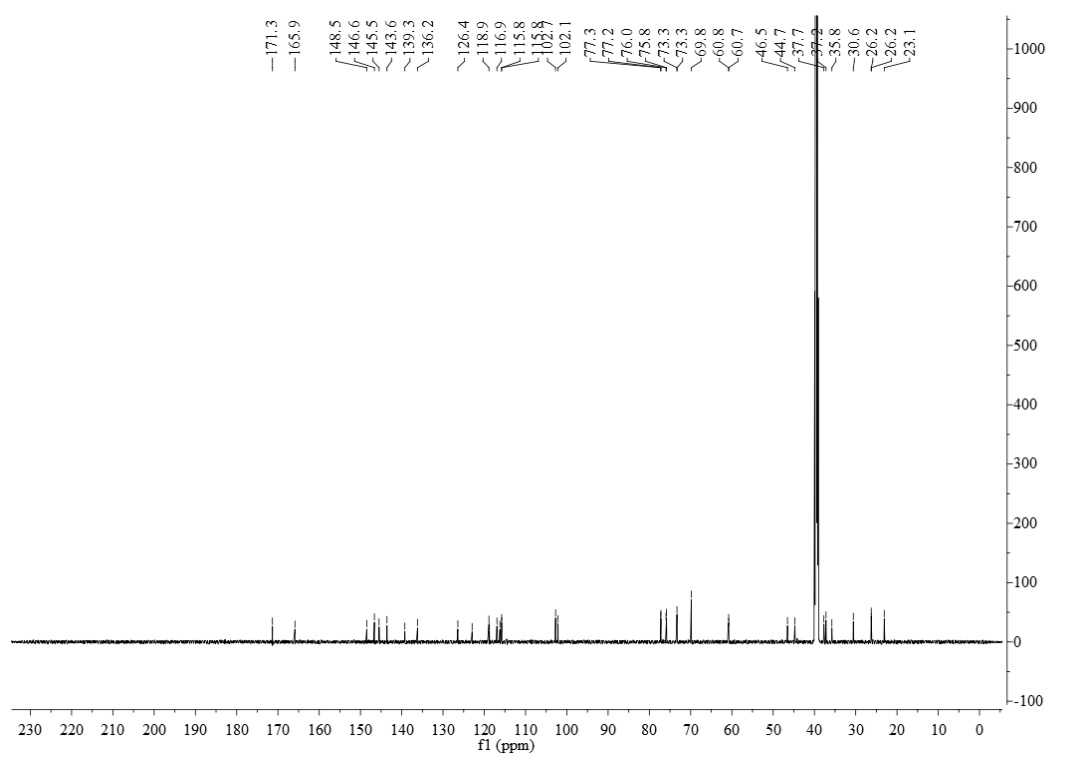

Figure S28. ${ }^{13} \mathrm{C}$ NMR spectrum of lycibarbarspermidine E (5) in DMSO- $d_{6}$ at $150 \mathrm{MHz}$ 


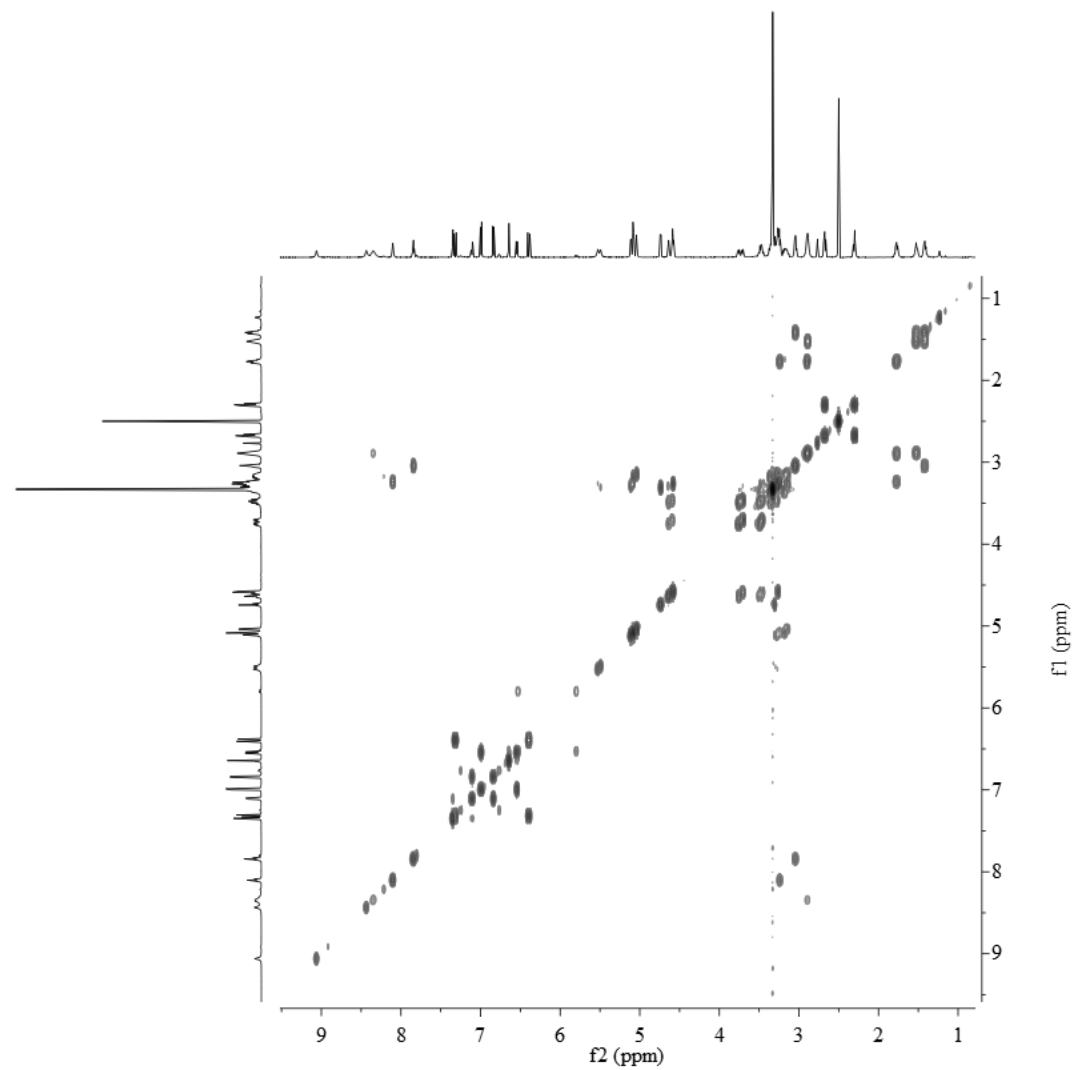

Figure S29. ${ }^{1} \mathrm{H}-{ }^{1} \mathrm{H}$ COSY spectrum of lycibarbarspermidine E (5) in DMSO- $d_{6}$ at $600 \mathrm{MHz}$

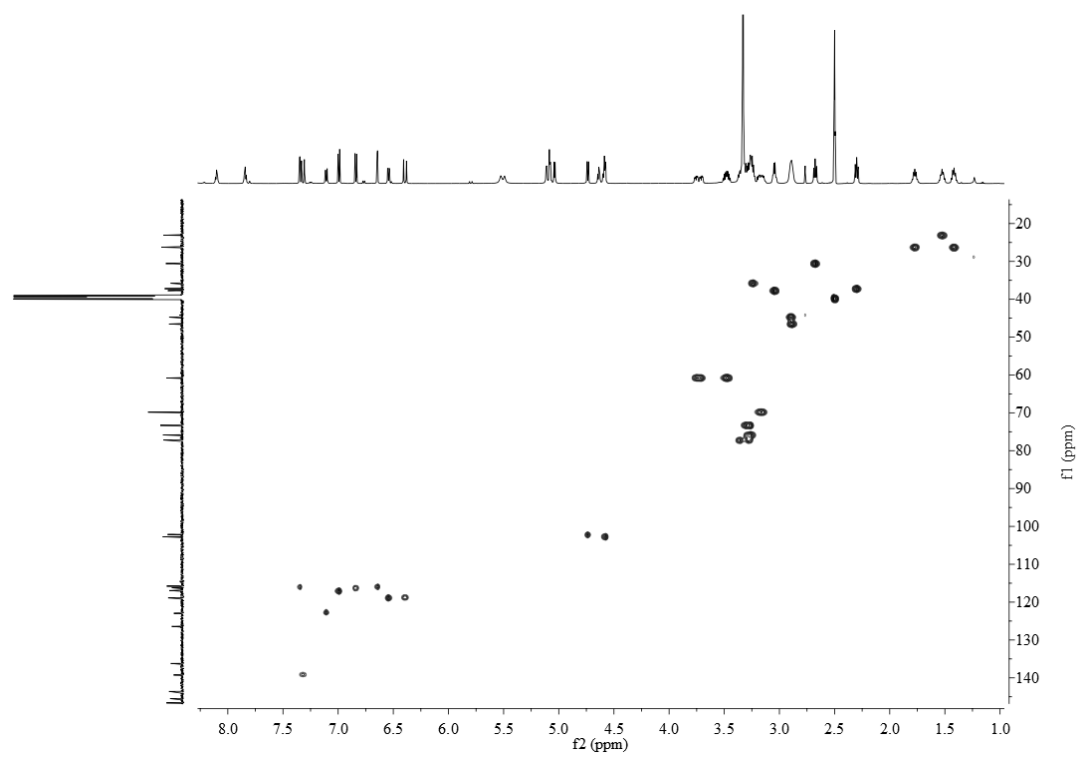

Figure S30. HSQC spectrum of lycibarbarspermidine E (5) in DMSO- $d_{6}$ at $600 \mathrm{MHz}$ 


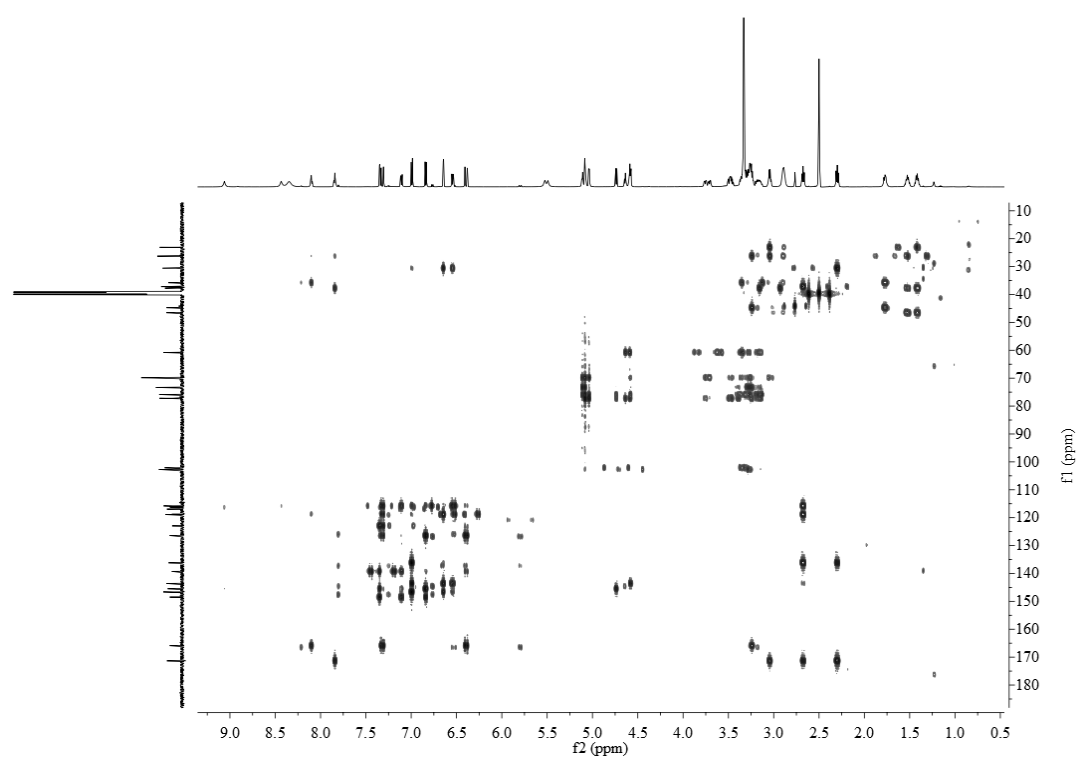

Figure S31. HMBC spectrum of lycibarbarspermidine E (5) in DMSO- $d_{6}$ at $600 \mathrm{MHz}$

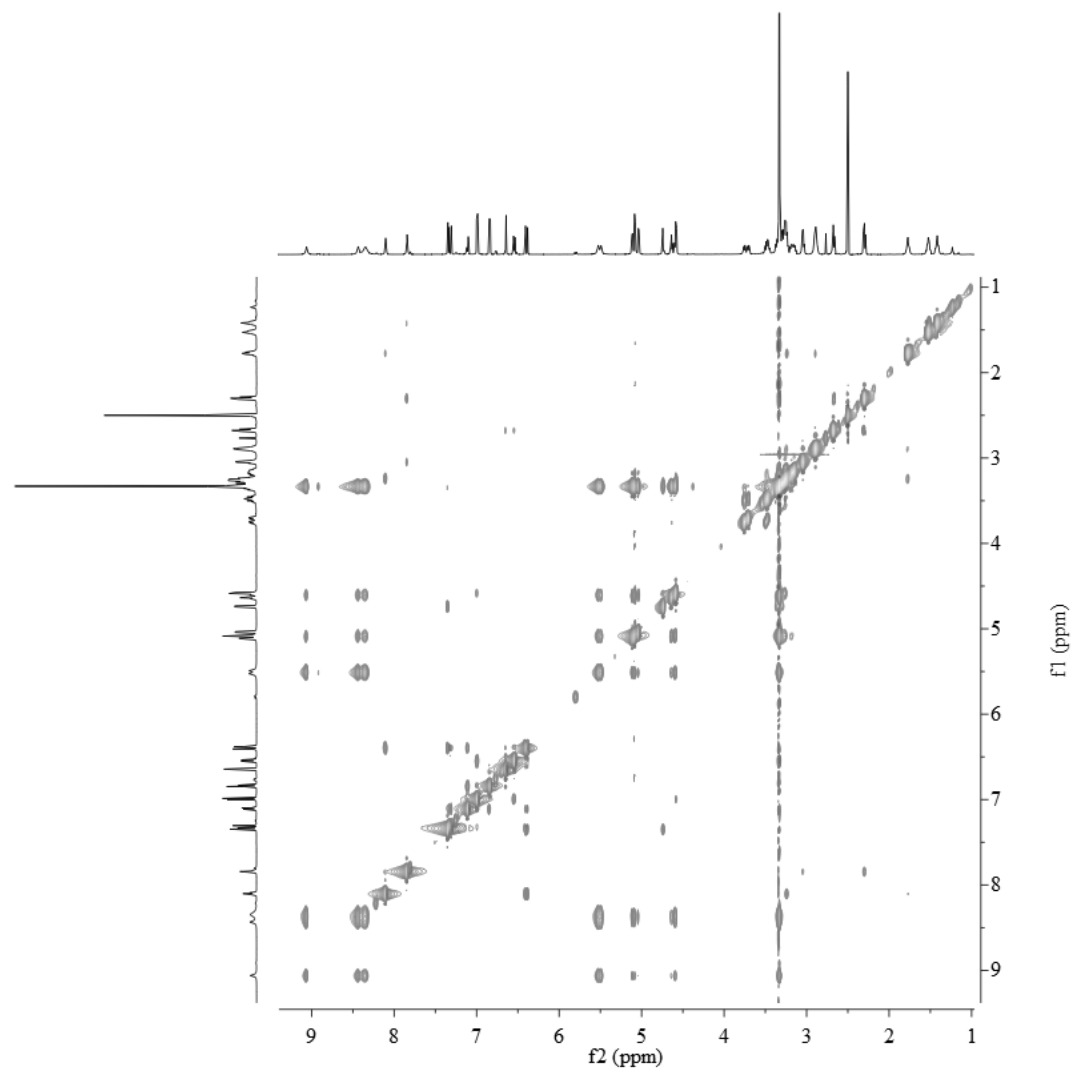

Figure S32. NOESY spectrum of lycibarbarspermidine E (5) in DMSO- $d_{6}$ at $600 \mathrm{MHz}$ 


\section{The 1D and 2D NMR spectra of 6.}

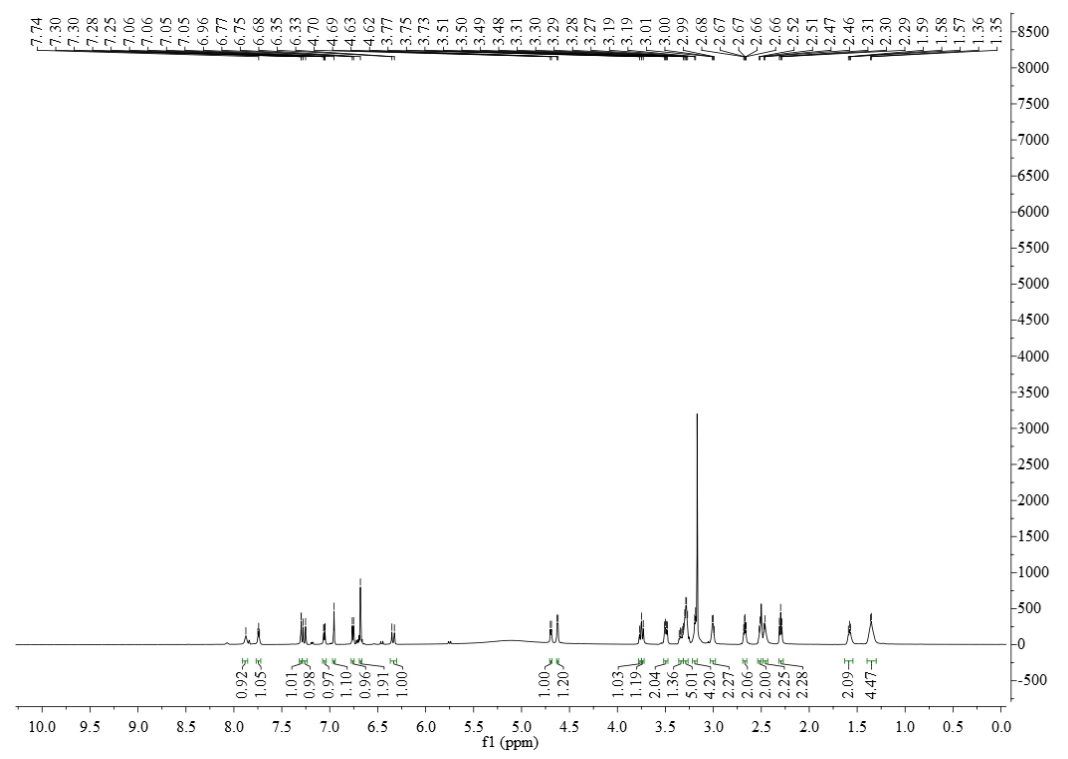

Figure S33. ${ }^{1} \mathrm{H}$ NMR spectrum of lycibarbarspermidine F (6) in DMSO- $d_{6}$ at $600 \mathrm{MHz}$

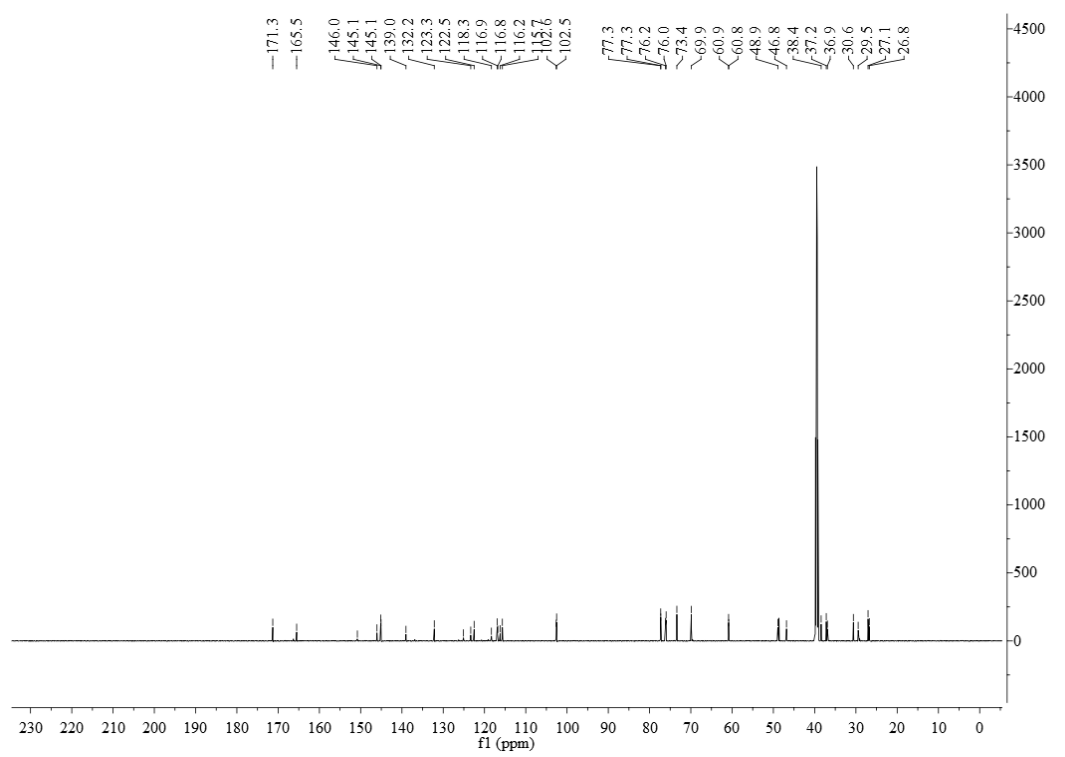

Figure S34. ${ }^{13} \mathrm{C}$ NMR spectrum of lycibarbarspermidine F (6) in DMSO- $d_{6}$ at $150 \mathrm{MHz}$ 


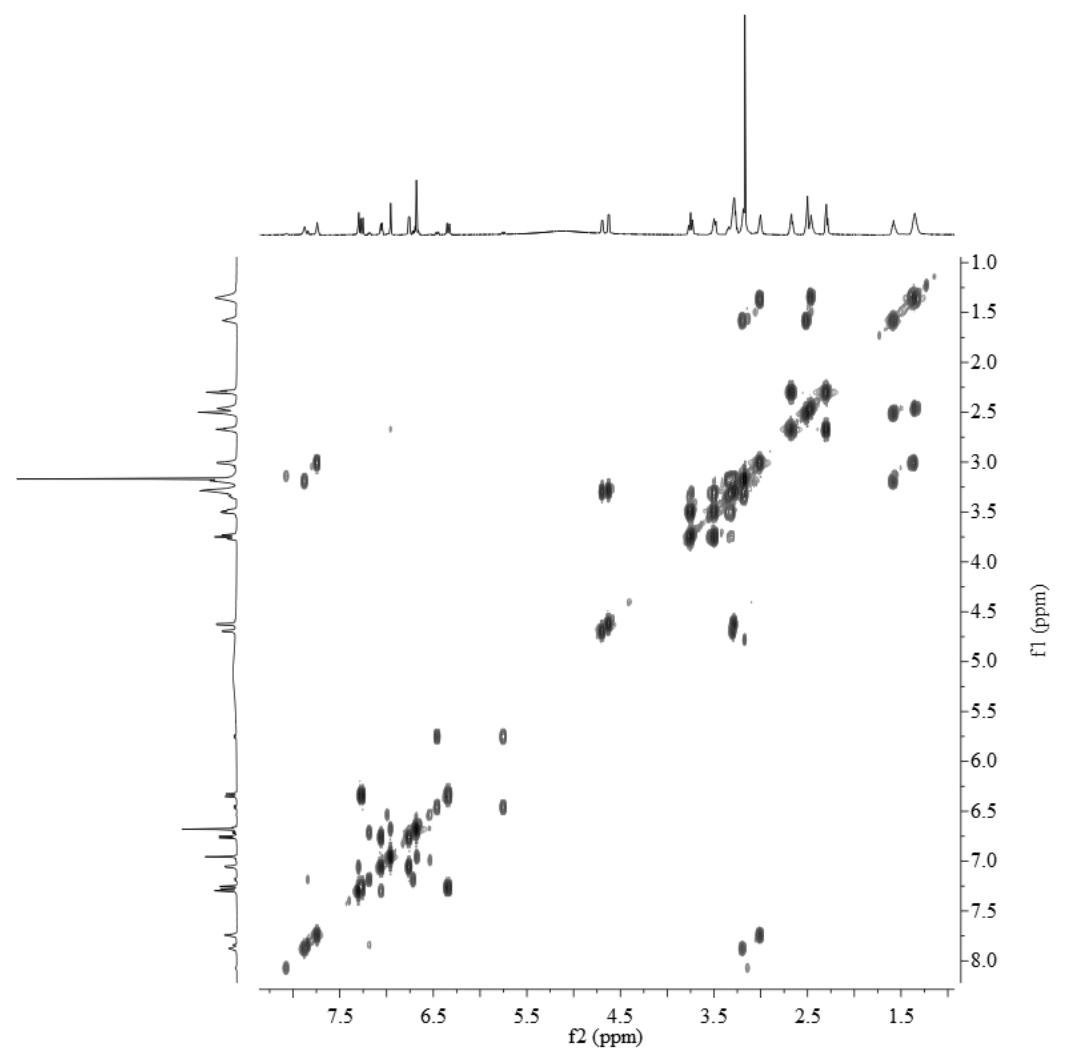

Figure S35. ${ }^{1} \mathrm{H}-{ }^{1} \mathrm{H}$ COSY spectrum of lycibarbarspermidine F (6) in DMSO- $d_{6}$ at $600 \mathrm{MHz}$

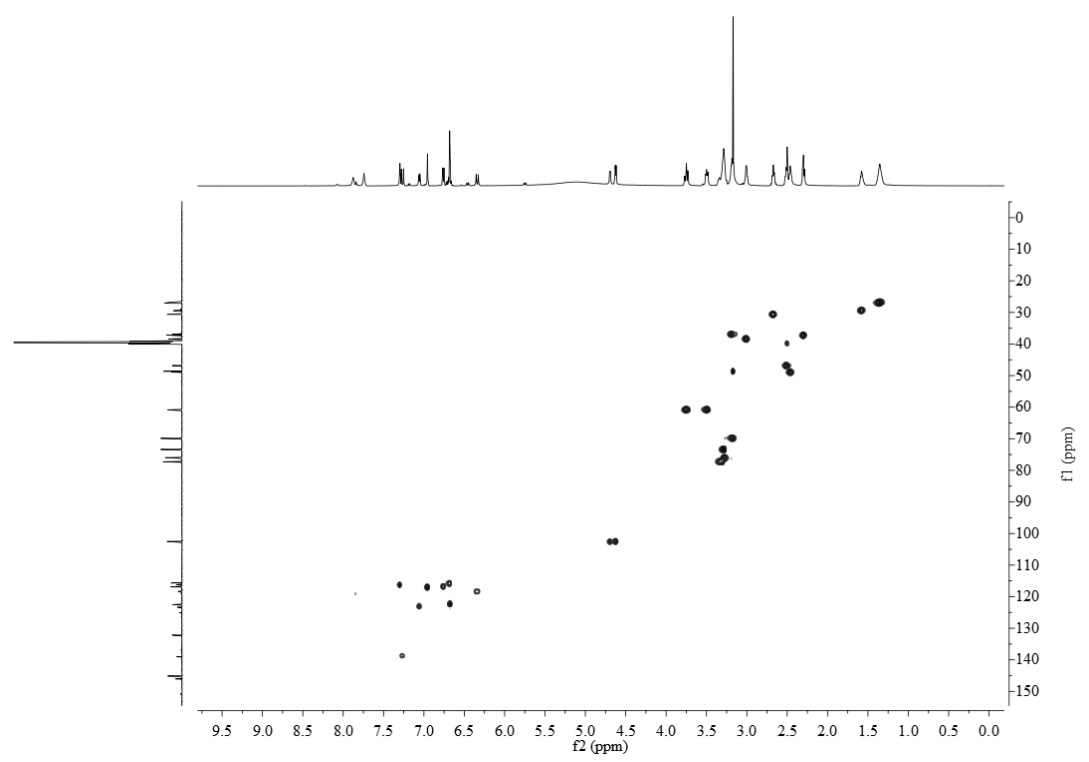

Figure S36. HSQC spectrum of lycibarbarspermidine F (6) in DMSO- $d_{6}$ at $600 \mathrm{MHz}$ 


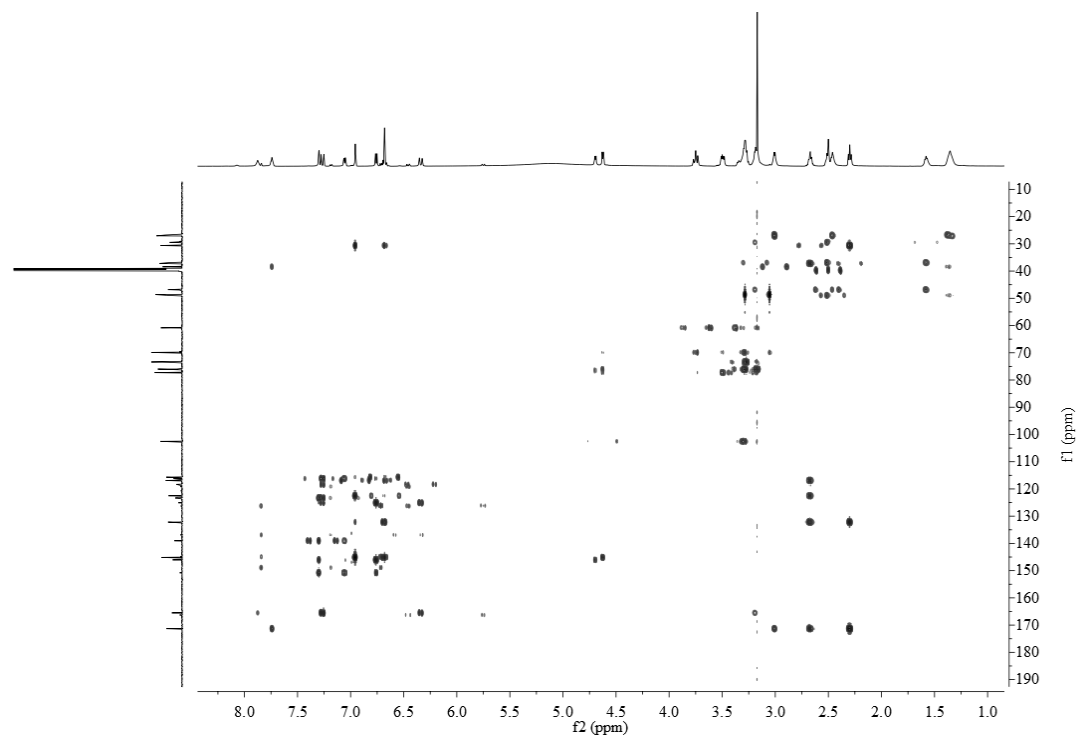

Figure S37. HMBC spectrum of lycibarbarspermidine F (6) in DMSO- $d_{6}$ at $600 \mathrm{MHz}$

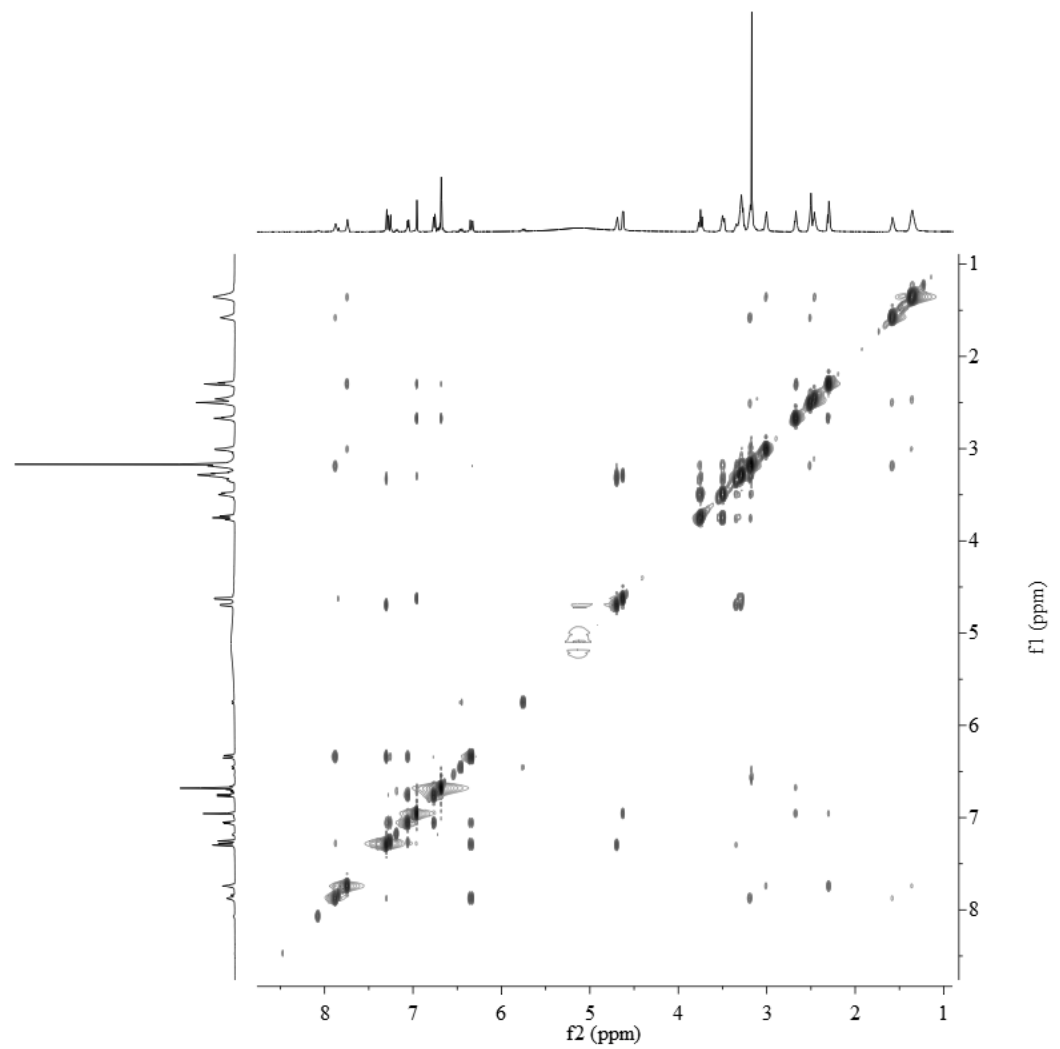

Figure S38. NOESY spectrum of lycibarbarspermidine F (6) in DMSO- $d_{6}$ at $600 \mathrm{MHz}$ 


\section{The 1D and 2D NMR spectra of 7.}

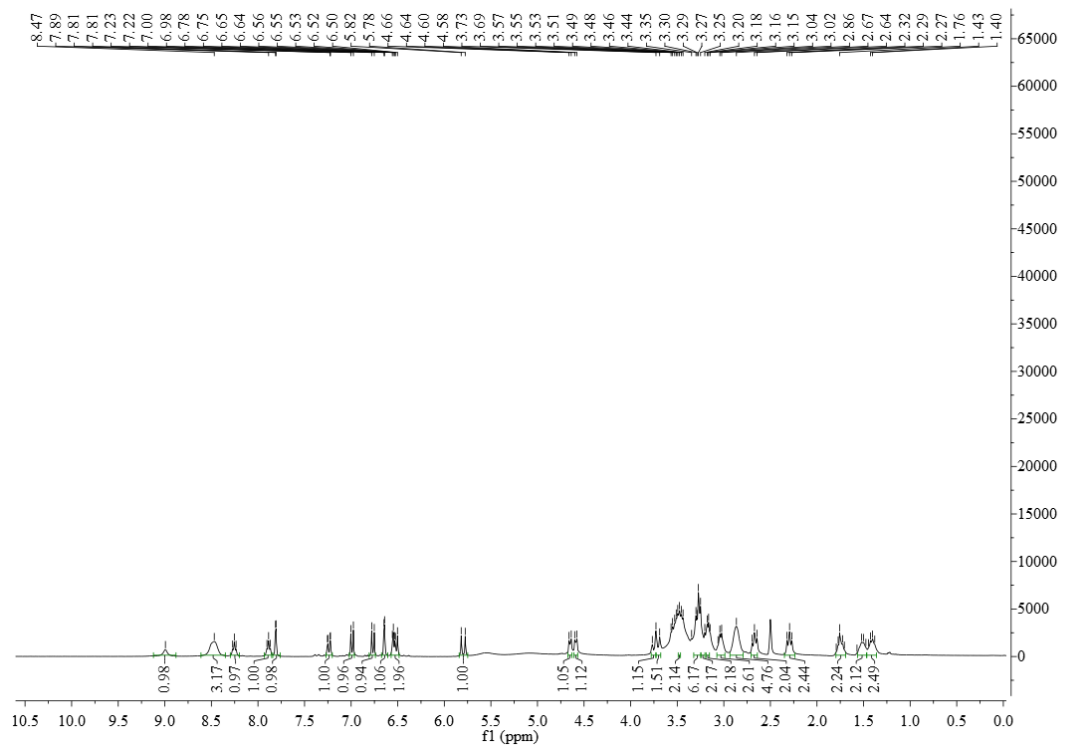

Figure S39. ${ }^{1} \mathrm{H}$ NMR spectrum of lycibarbarspermidine G (7) in DMSO- $d_{6}$ at $300 \mathrm{MHz}$

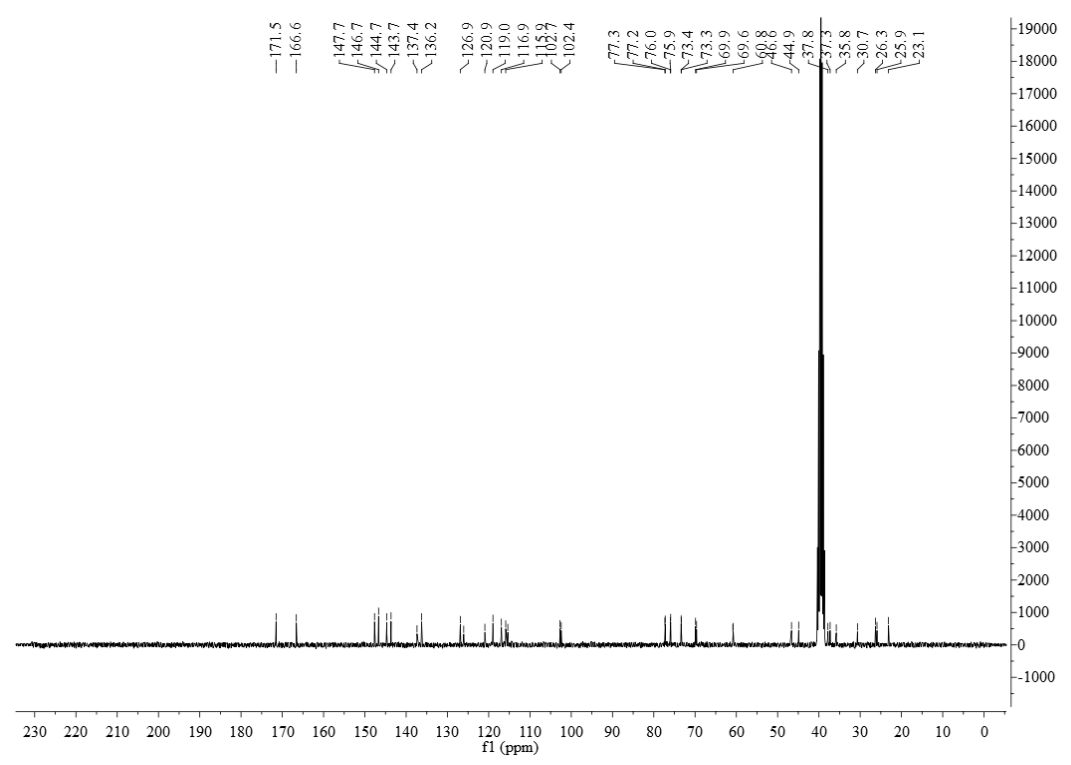

Figure S40. ${ }^{13} \mathrm{C}$ NMR spectrum of lycibarbarspermidine G (7) in DMSO- $d_{6}$ at $75 \mathrm{MHz}$ 


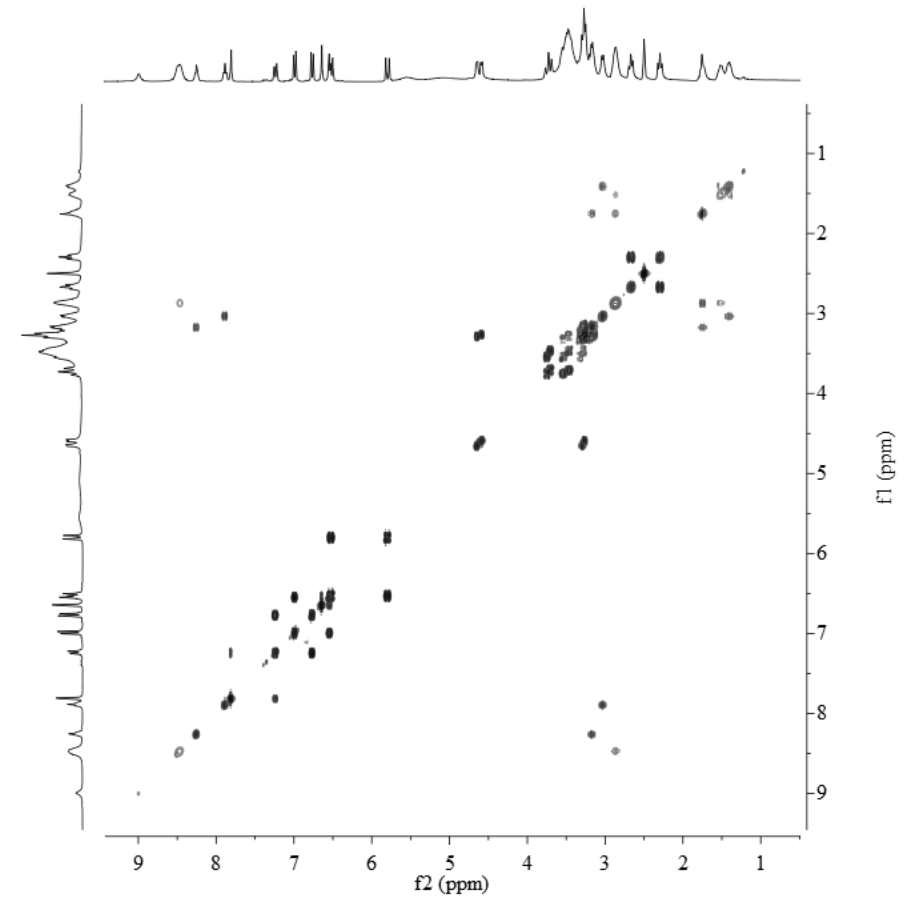

Figure S41. ${ }^{1} \mathrm{H}-{ }^{1} \mathrm{H}$ COSY spectrum of lycibarbarspermidine G (7) in DMSO- $d_{6}$ at $300 \mathrm{MHz}$

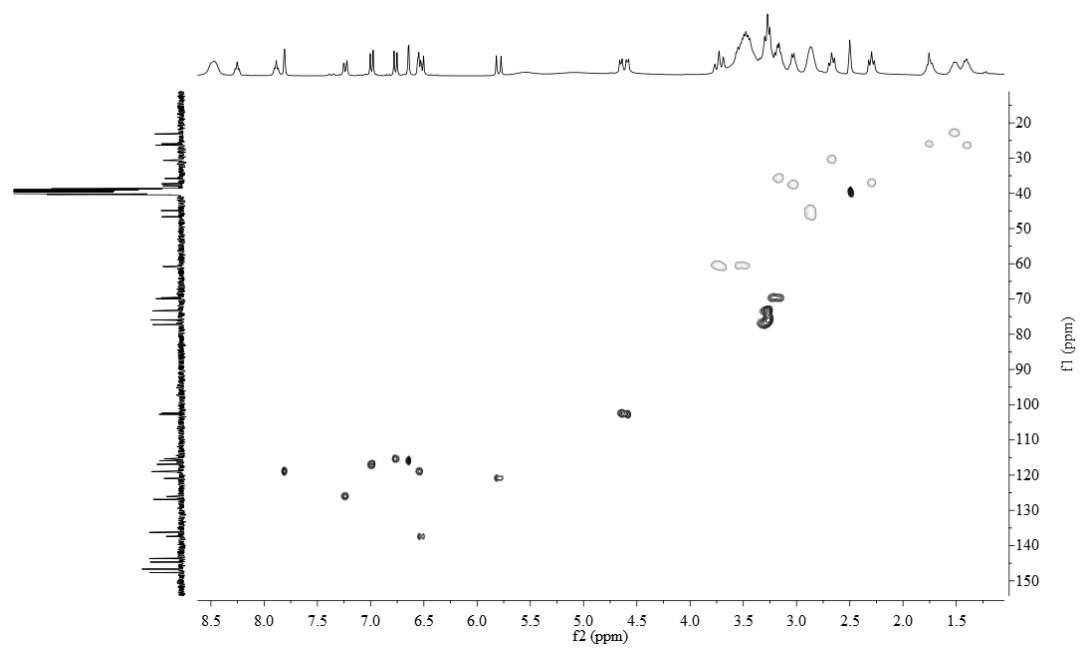

Figure S42. HSQC spectrum of lycibarbarspermidine G (7) in DMSO- $d_{6}$ at $300 \mathrm{MHz}$ 


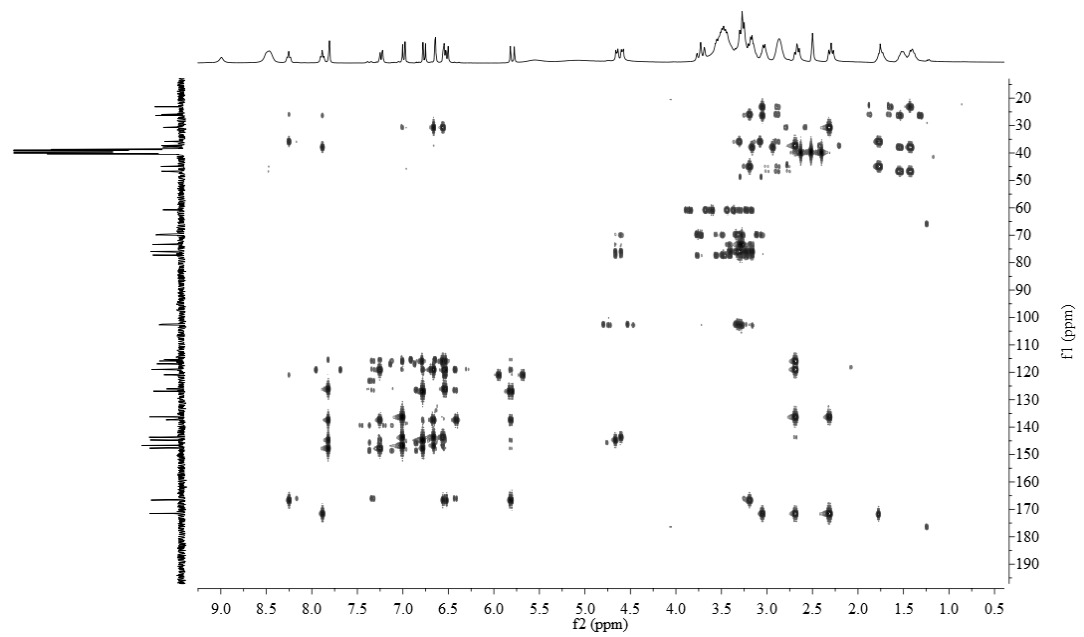

Figure S43. HMBC spectrum of lycibarbarspermidine G (7) in DMSO- $d_{6}$ at $600 \mathrm{MHz}$

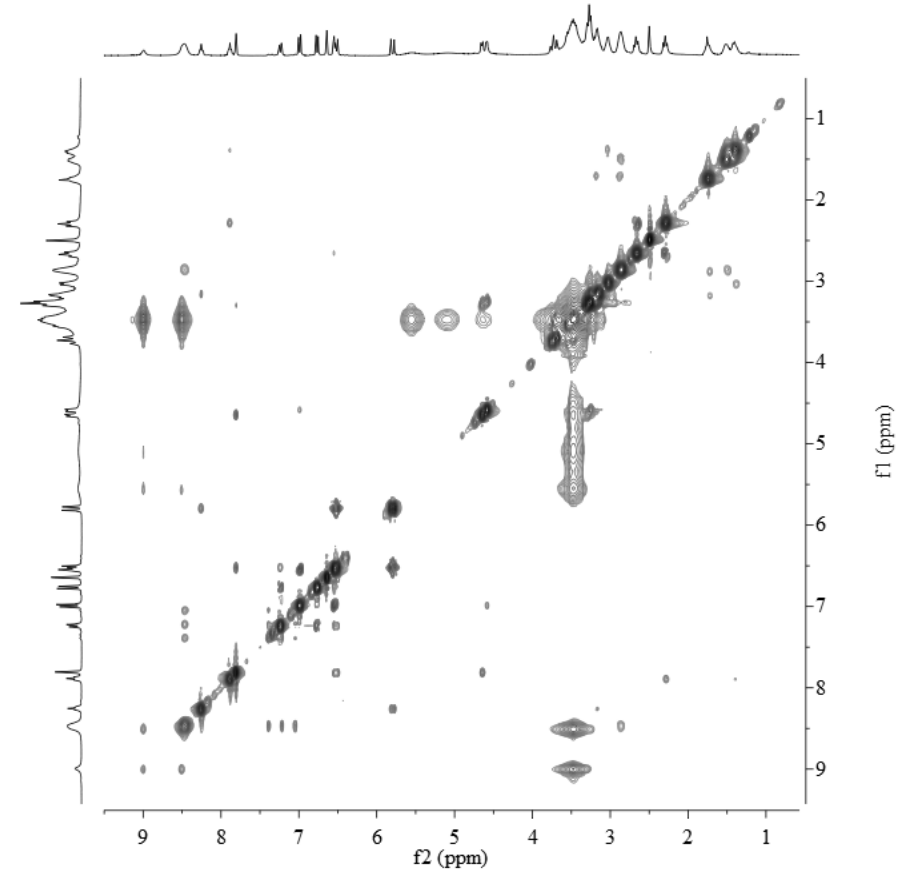

Figure S44. NOESY spectrum of lycibarbarspermidine G (7) in DMSO- $d_{6}$ at $300 \mathrm{MHz}$ 


\section{The 1D and 2D NMR spectra of 8/9.}

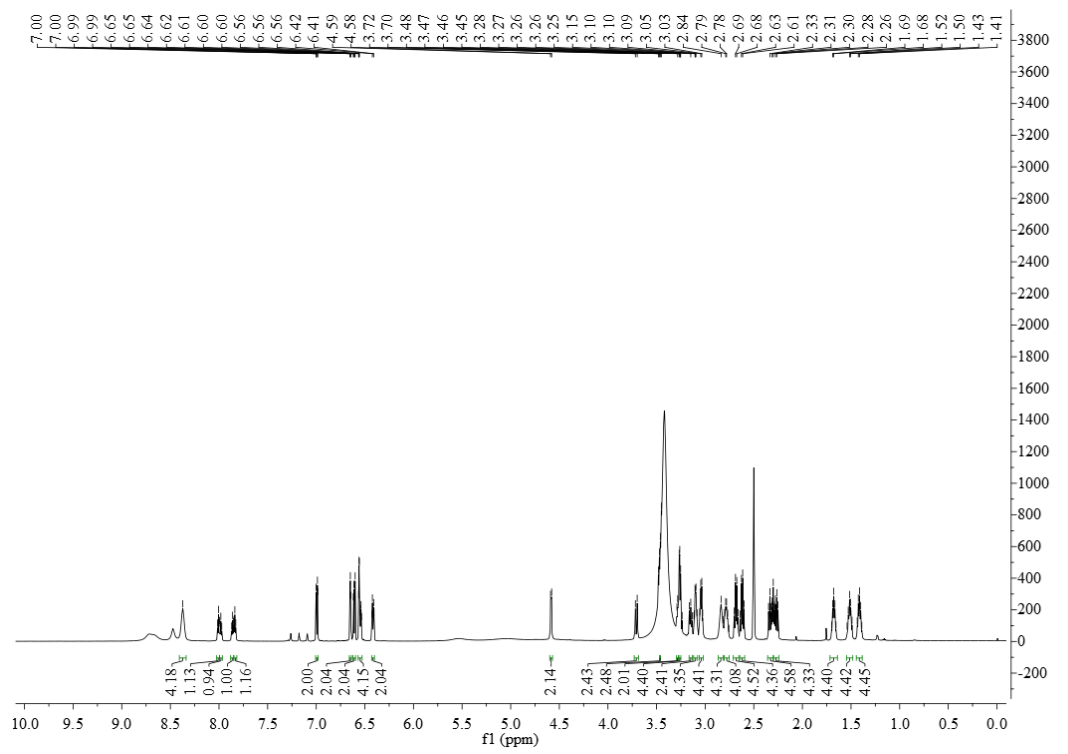

Figure S45. ${ }^{1} \mathrm{H}$ NMR spectrum of lycibarbarspermidines H/I (8/9) in DMSO- $d_{6}$ at $600 \mathrm{MHz}$

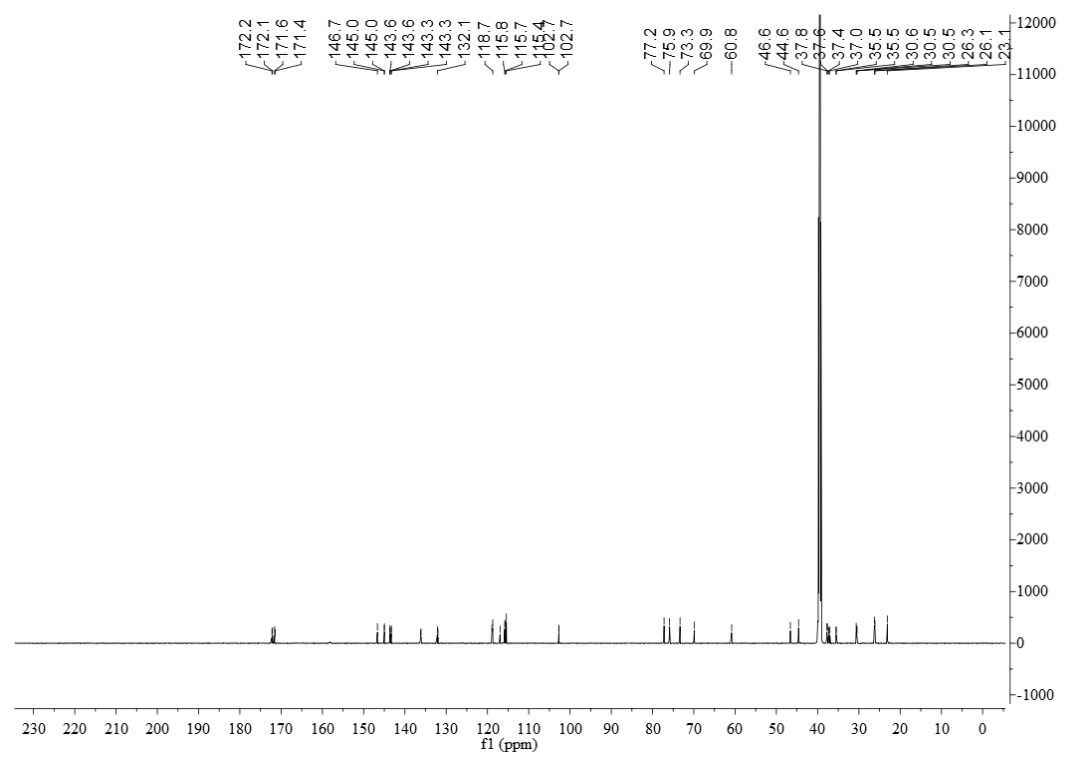

Figure S46. ${ }^{13} \mathrm{C}$ NMR spectrum of lycibarbarspermidines H/I (8/9) in DMSO- $d_{6}$ at $150 \mathrm{MHz}$ 


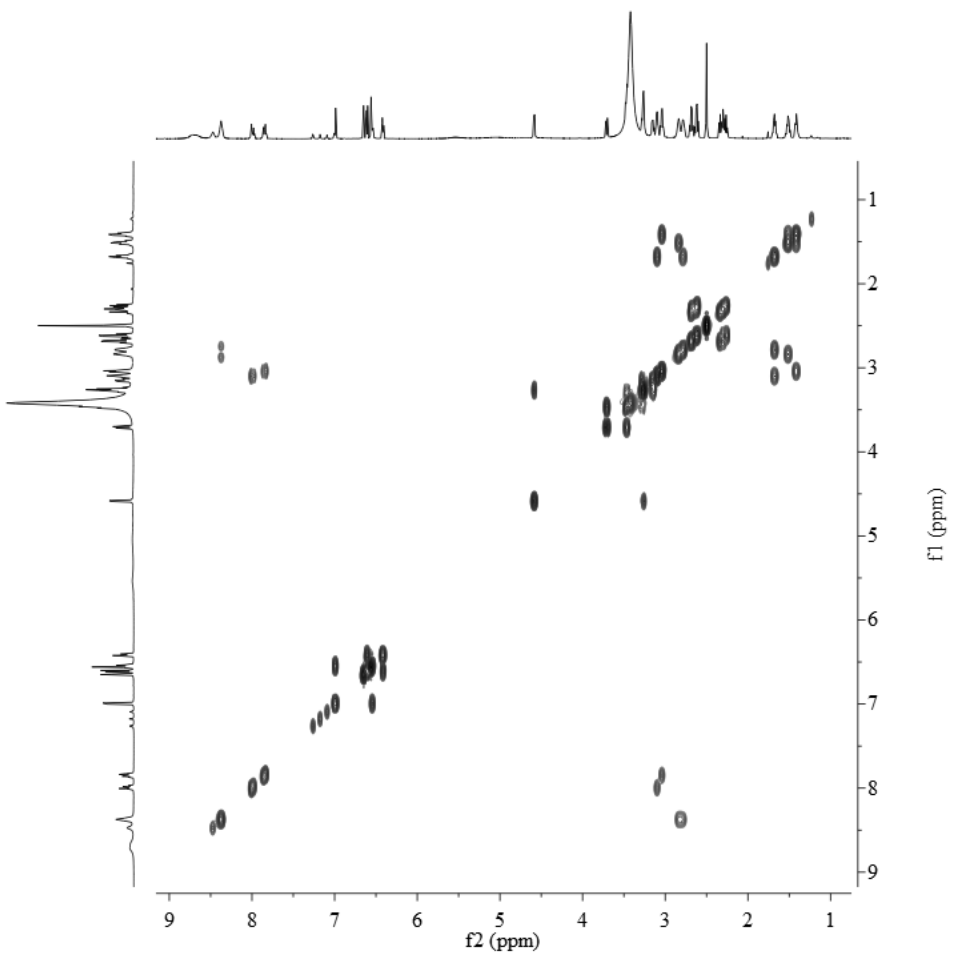

Figure S47. ${ }^{1} \mathrm{H}-{ }^{1} \mathrm{H}$ COSY spectrum of lycibarbarspermidines $\mathrm{H} / \mathrm{I}(\mathbf{8} / \mathbf{9})$ in DMSO- $d_{6}$ at $600 \mathrm{MHz}$

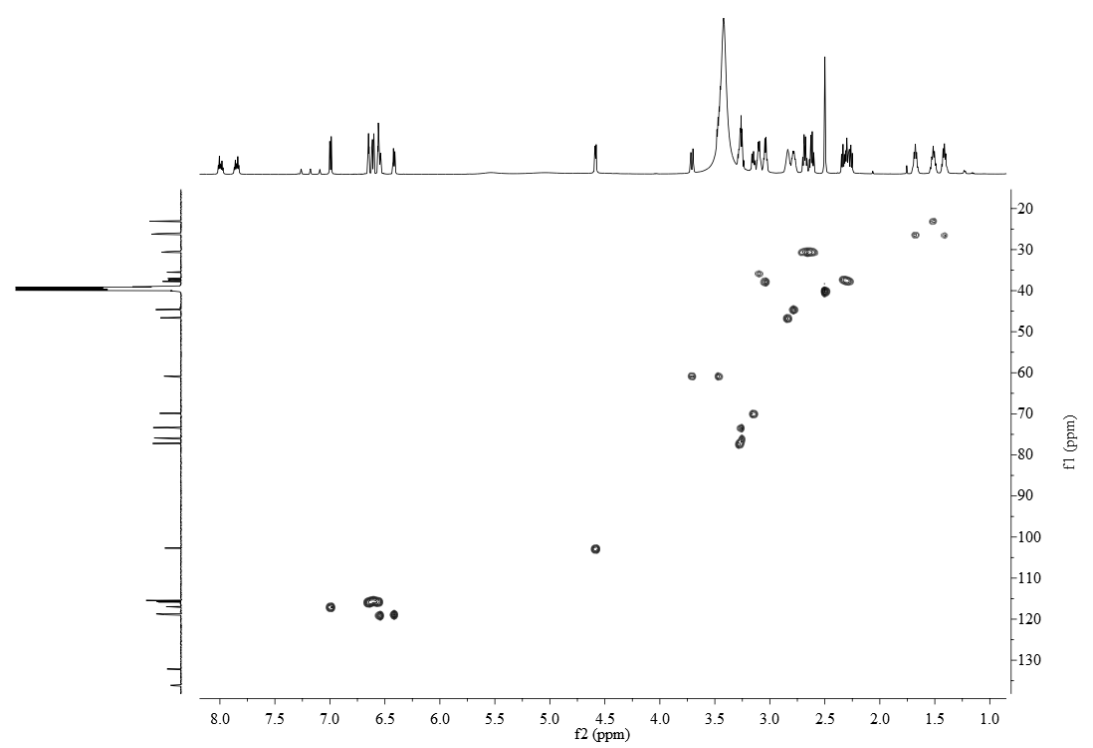

Figure S48. HSQC spectrum of lycibarbarspermidines H/I (8/9) in DMSO- $d_{6}$ at $600 \mathrm{MHz}$ 


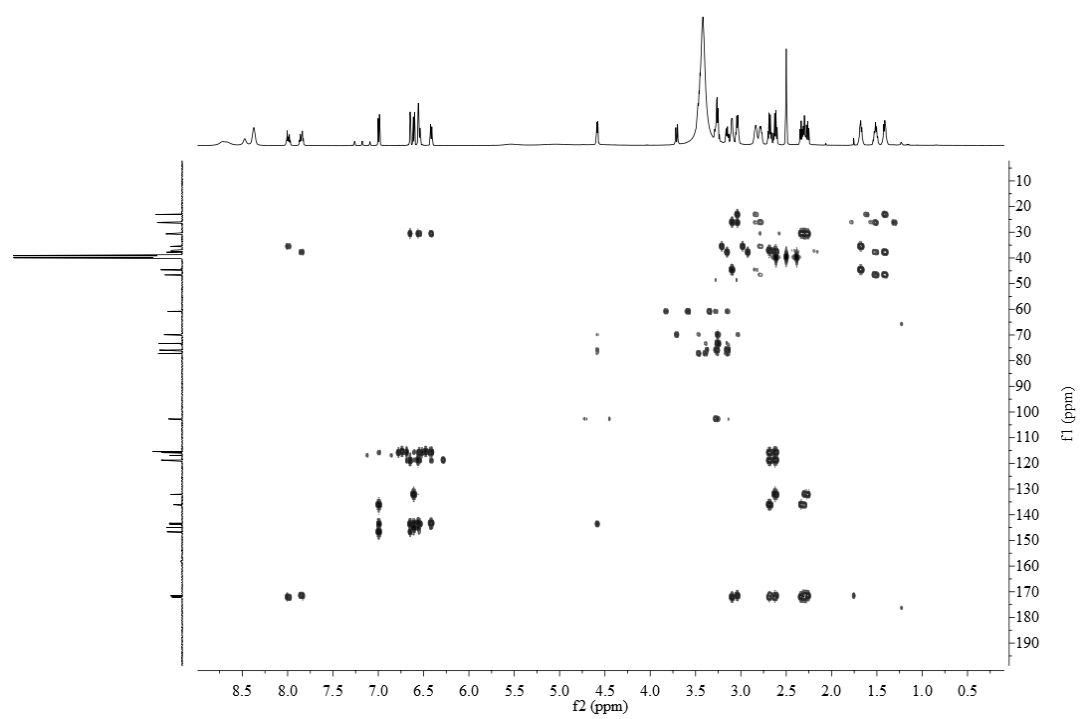

Figure S49. HMBC spectrum of lycibarbarspermidines H/I (8/9) in DMSO- $d_{6}$ at $600 \mathrm{MHz}$

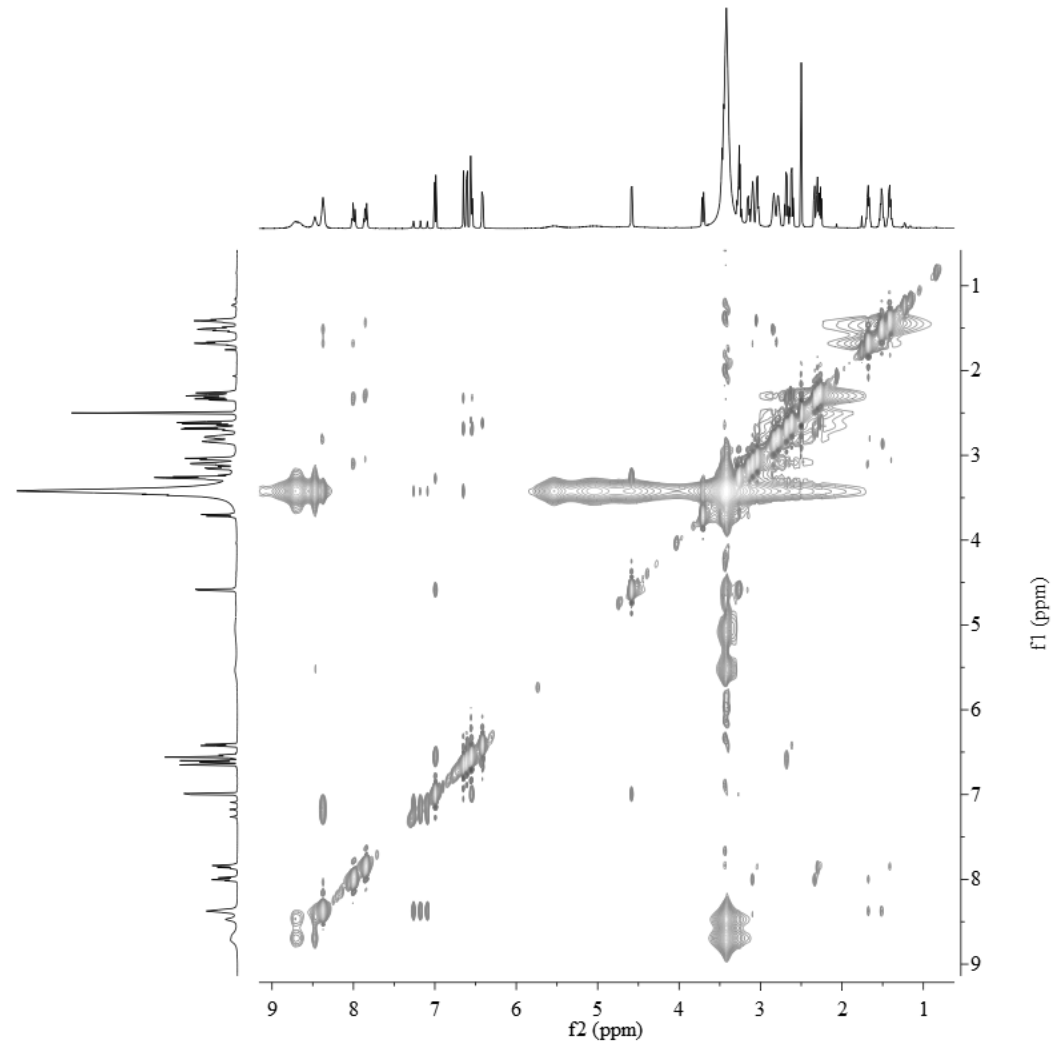

Figure S50. NOESY spectrum of lycibarbarspermidines H/I (8/9) in DMSO- $d_{6}$ at $600 \mathrm{MHz}$ 


\section{The 1D and 2D NMR spectra of 10.}

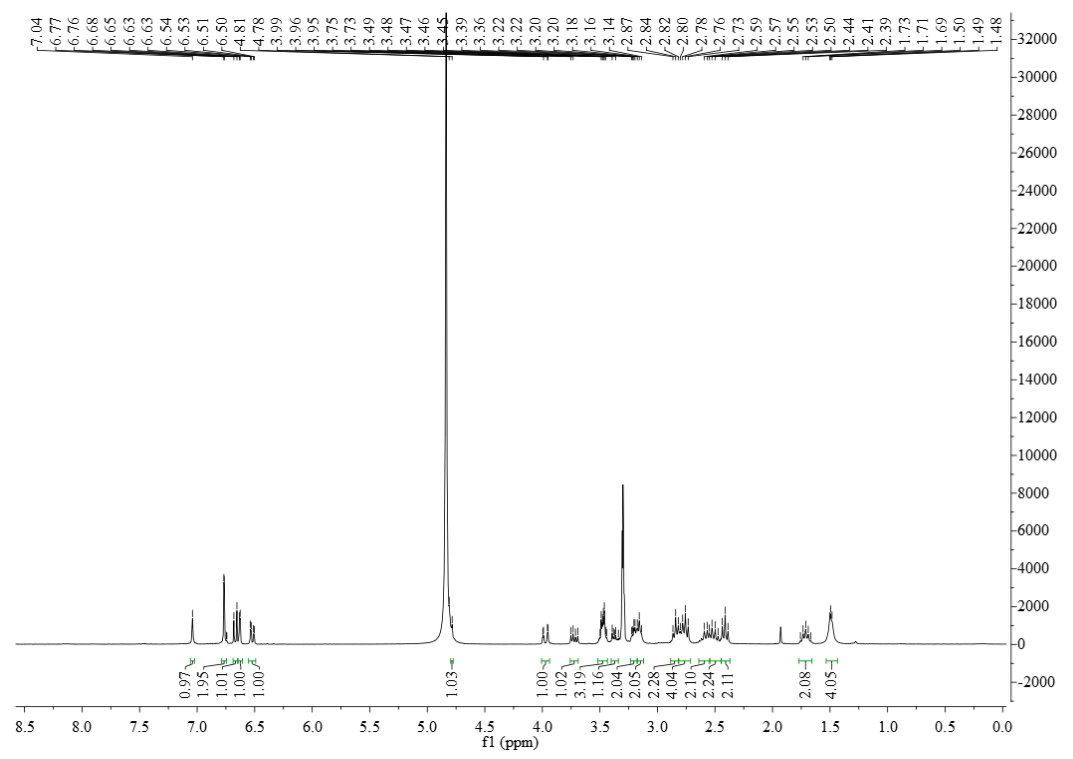

Figure S51. ${ }^{1} \mathrm{H}$ NMR spectrum of lycibarbarspermidine $\mathrm{J}(\mathbf{1 0})$ in $\mathrm{CD}_{3} \mathrm{OD}$ at $300 \mathrm{MHz}$

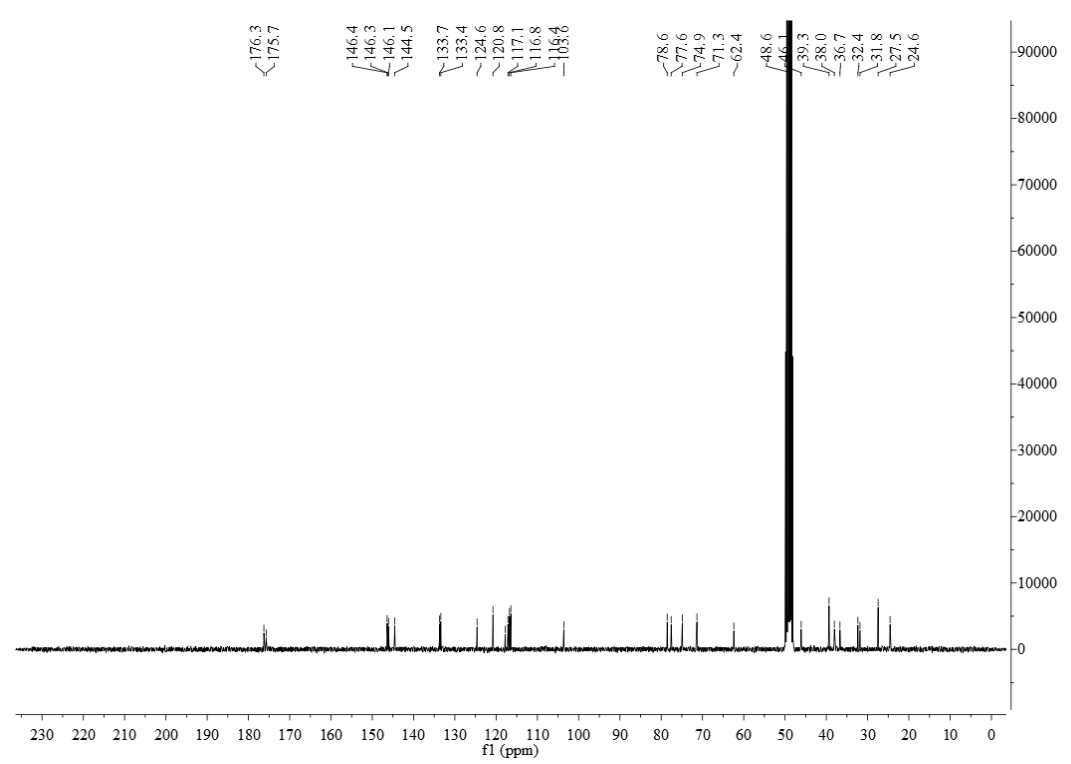

Figure S52. ${ }^{13} \mathrm{C}$ NMR spectrum of lycibarbarspermidine $\mathrm{J}(\mathbf{1 0})$ in $\mathrm{CD}_{3} \mathrm{OD}$ at $75 \mathrm{MHz}$ 


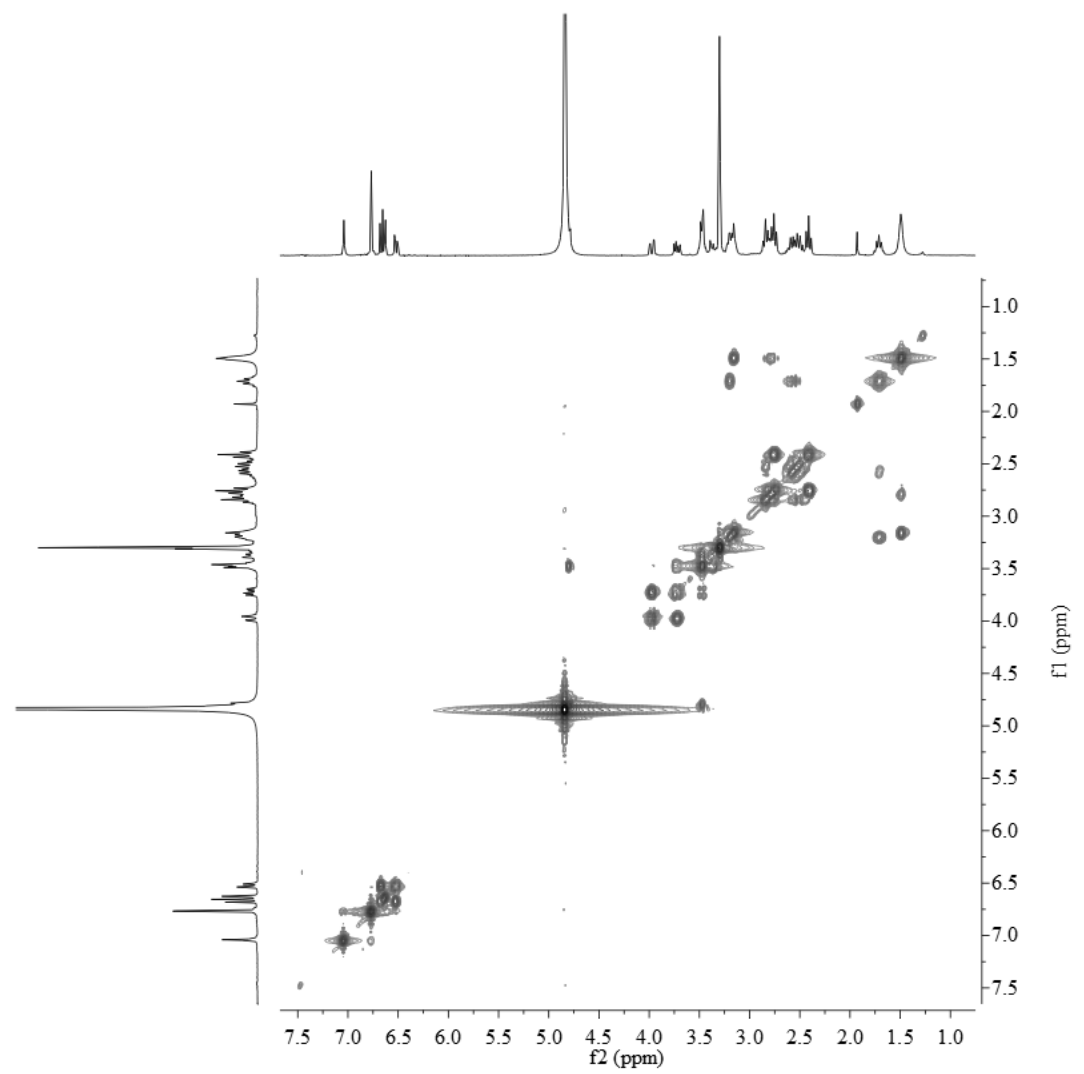

Figure S53. ${ }^{1} \mathrm{H}-{ }^{1} \mathrm{H}$ COSY spectrum of lycibarbarspermidine $\mathbf{J}(\mathbf{1 0})$ in $\mathrm{CD}_{3} \mathrm{OD}$ at $400 \mathrm{MHz}$

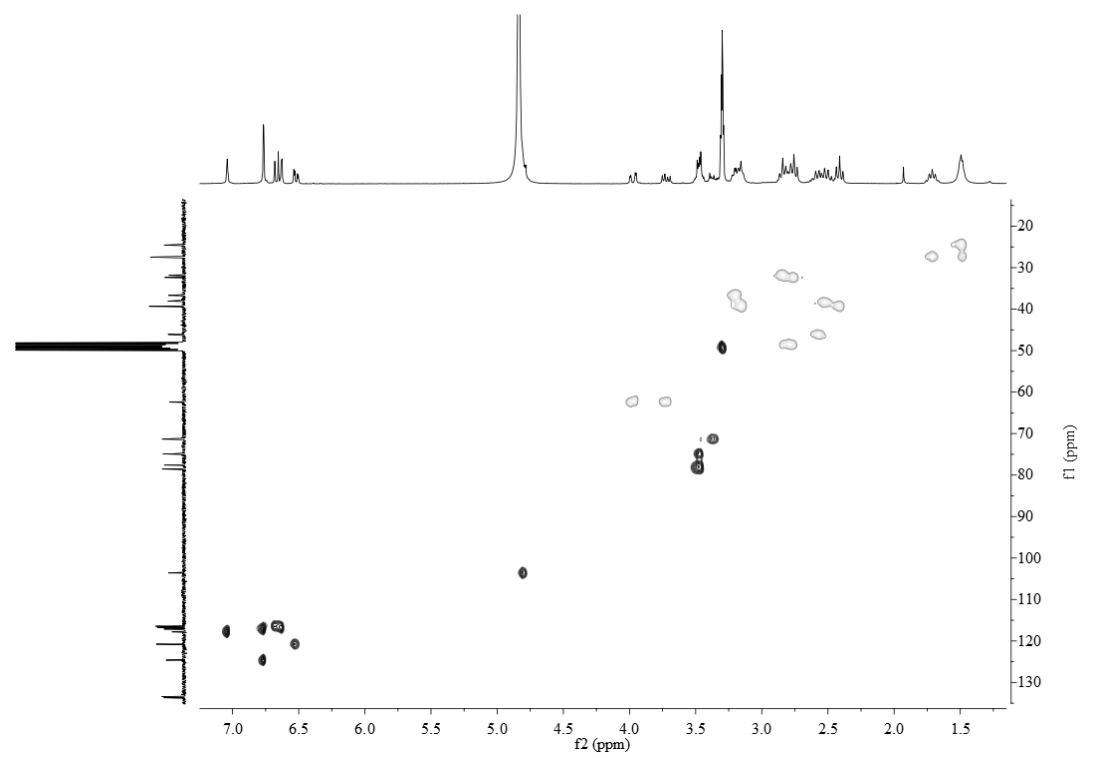

Figure S54. HSQC spectrum of lycibarbarspermidine $\mathrm{J}(\mathbf{1 0})$ in $\mathrm{CD}_{3} \mathrm{OD}$ at $300 \mathrm{MHz}$ 


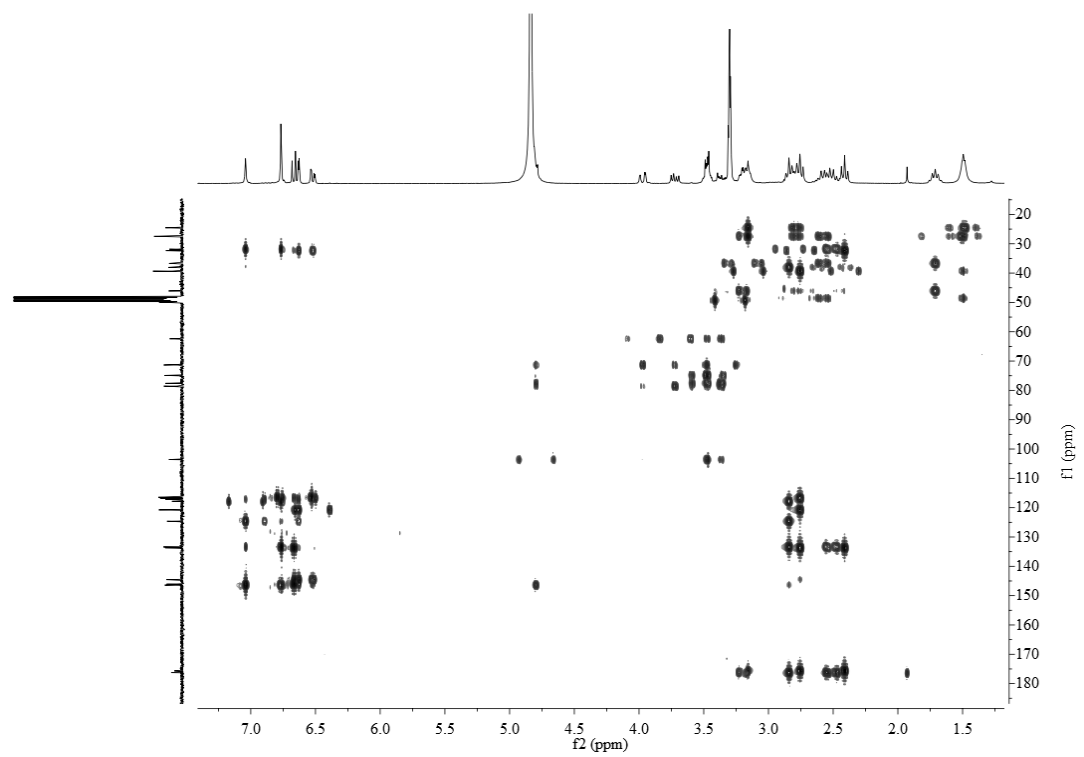

Figure S55. HMBC spectrum of lycibarbarspermidine $\mathrm{J}(\mathbf{1 0})$ in $\mathrm{CD}_{3} \mathrm{OD}$ at $600 \mathrm{MHz}$

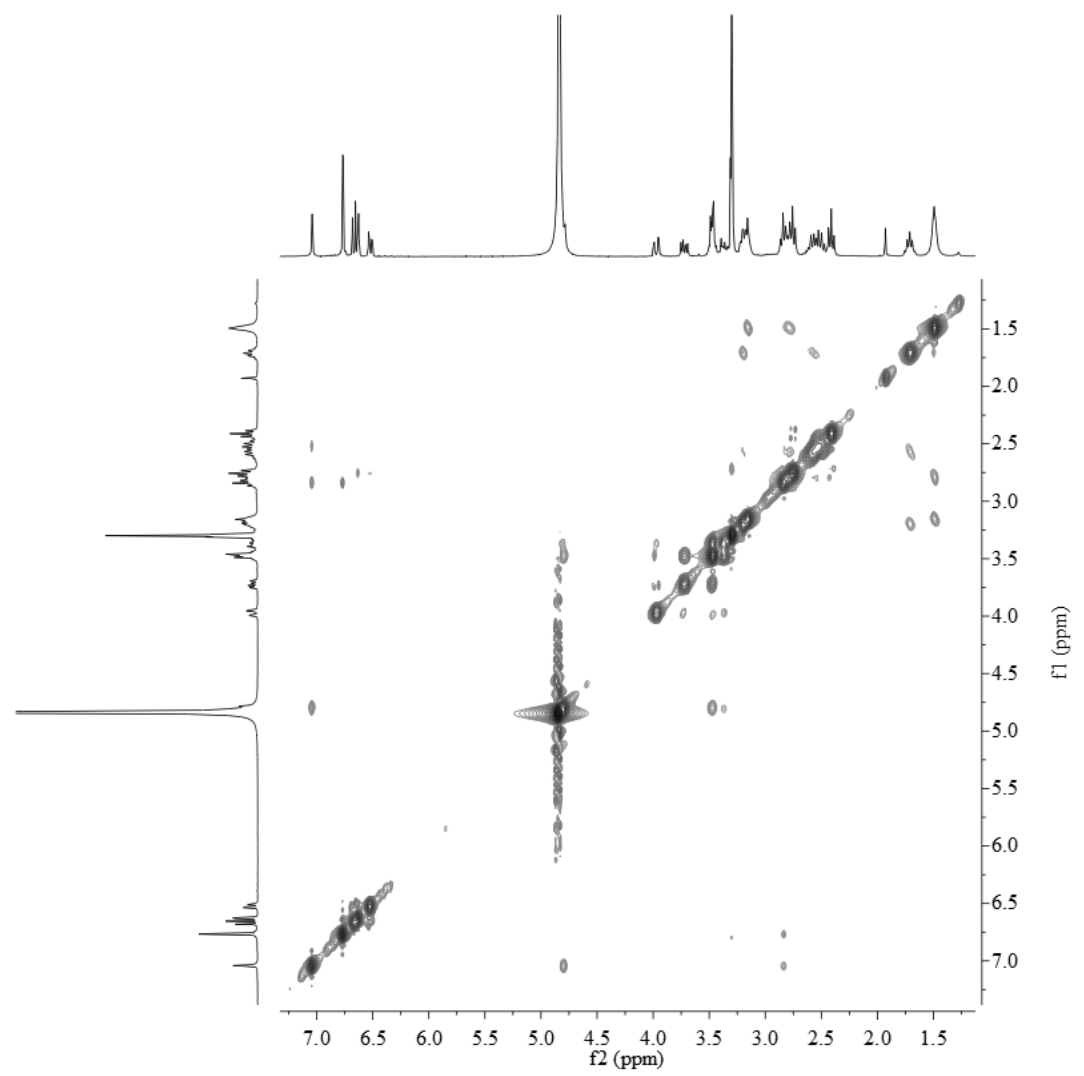

Figure S56. ROESY spectrum of lycibarbarspermidine $\mathrm{J}(\mathbf{1 0})$ in $\mathrm{CD}_{3} \mathrm{OD}$ at $400 \mathrm{MHz}$ 


\section{The 1D and 2D NMR spectra of 11.}

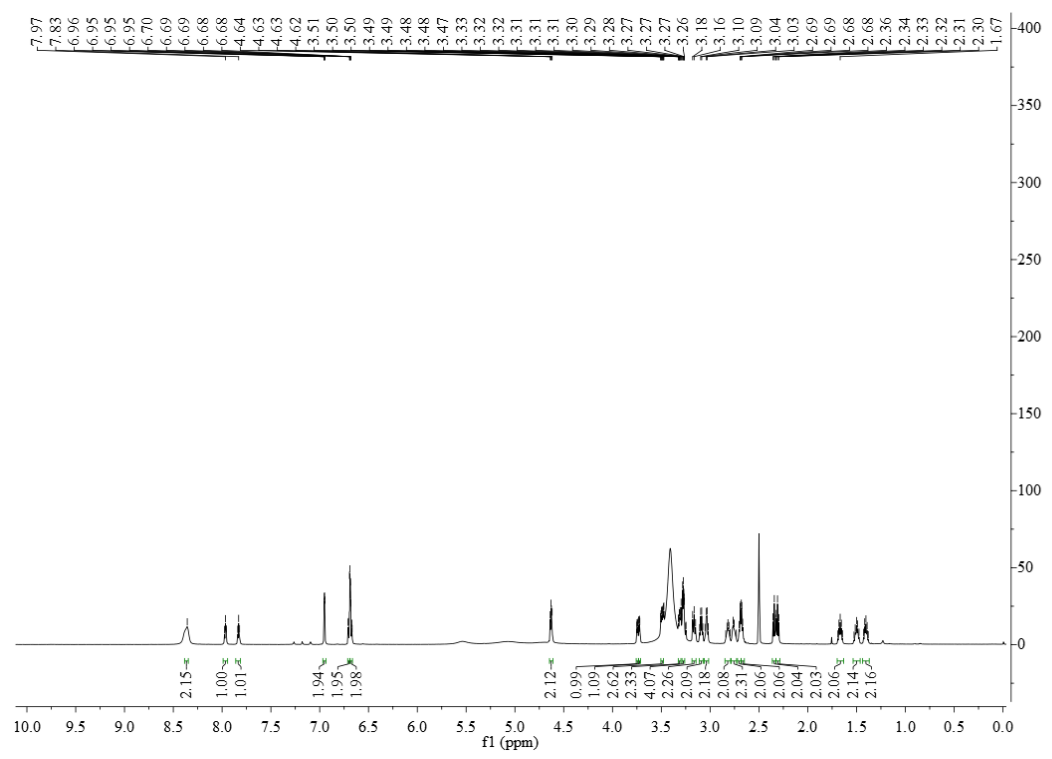

Figure S57. ${ }^{1} \mathrm{H}$ NMR spectrum of lycibarbarspermidine K (11) in DMSO- $d_{6}$ at $600 \mathrm{MHz}$

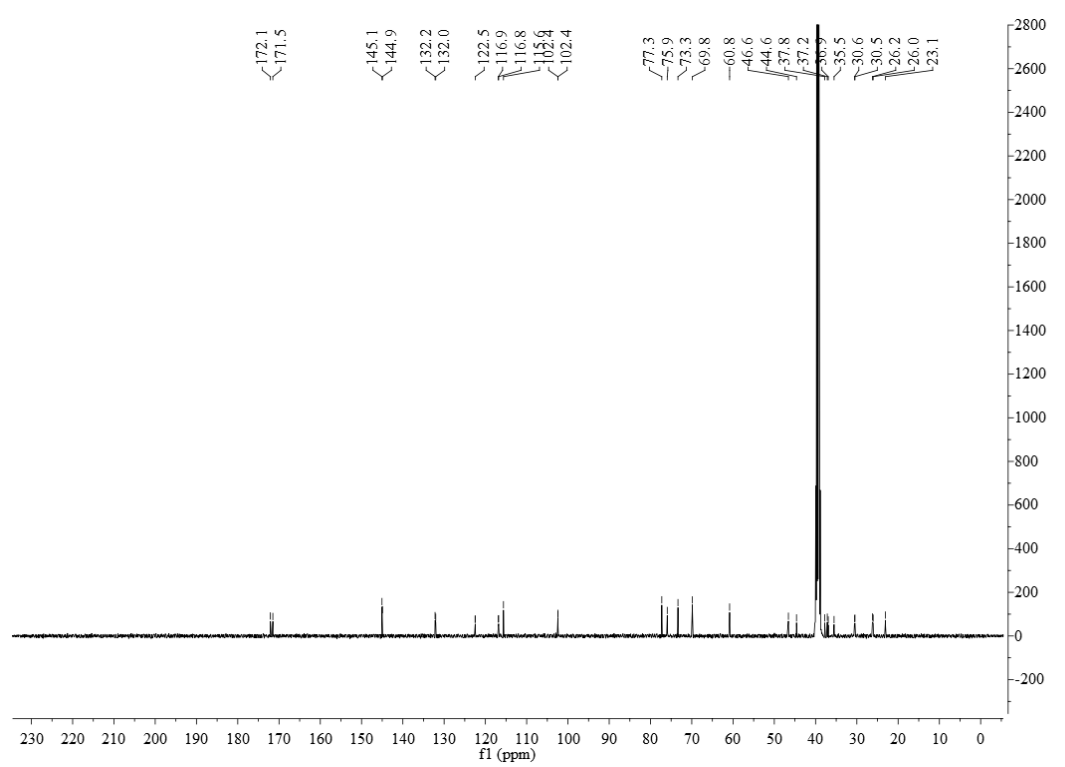

Figure S58. ${ }^{13} \mathrm{C}$ NMR spectrum of lycibarbarspermidine K (11) in DMSO- $d_{6}$ at $100 \mathrm{MHz}$ 


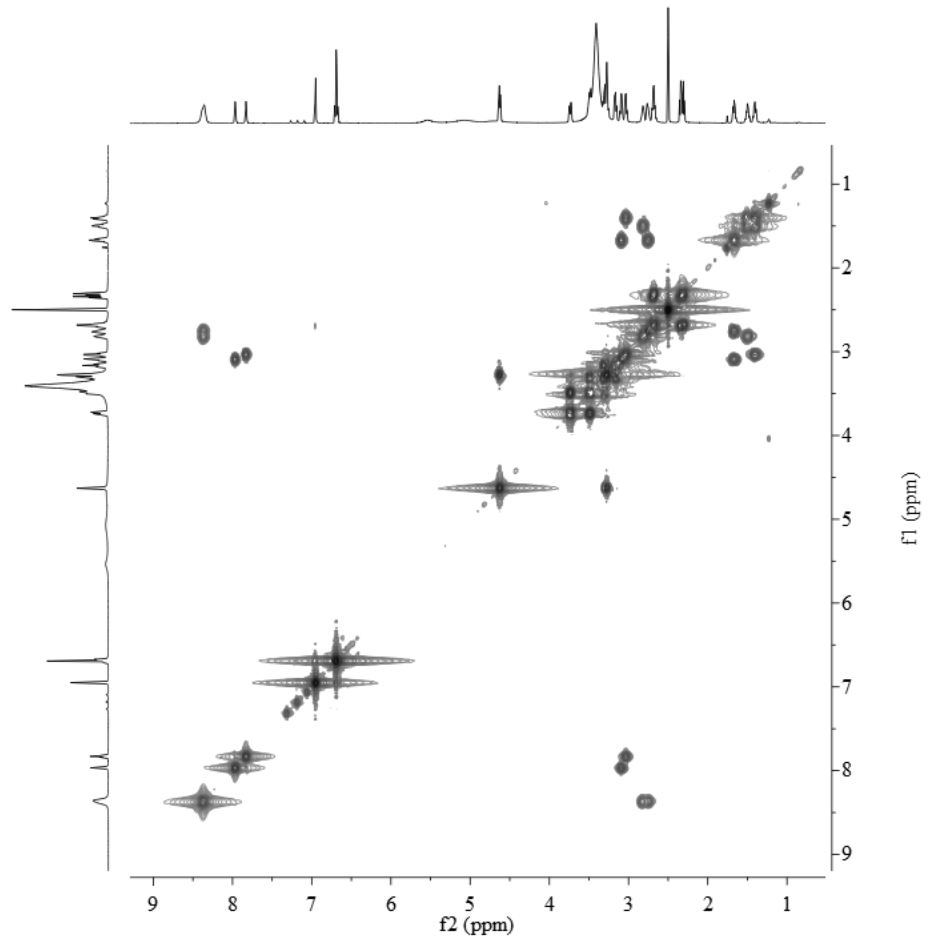

Figure S59. ${ }^{1} \mathrm{H}-{ }^{1} \mathrm{H}$ COSY spectrum of lycibarbarspermidine $\mathrm{K}(\mathbf{1 1})$ in DMSO- $d_{6}$ at $400 \mathrm{MHz}$

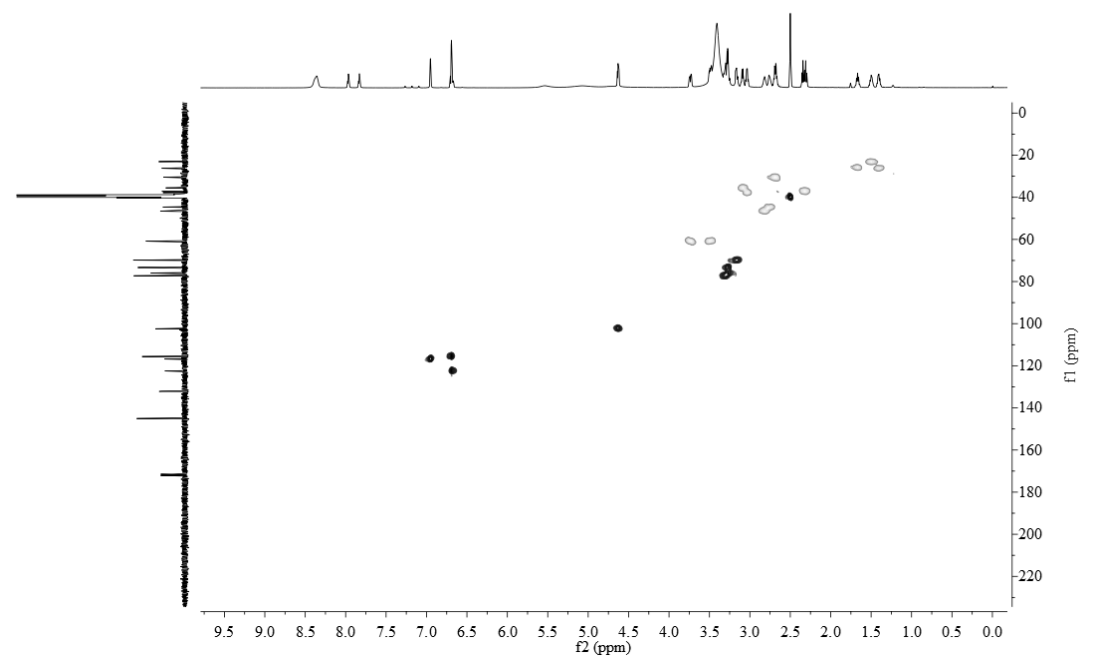

Figure S60. HSQC spectrum of lycibarbarspermidine K (11) in DMSO- $d_{6}$ at $300 \mathrm{MHz}$ 


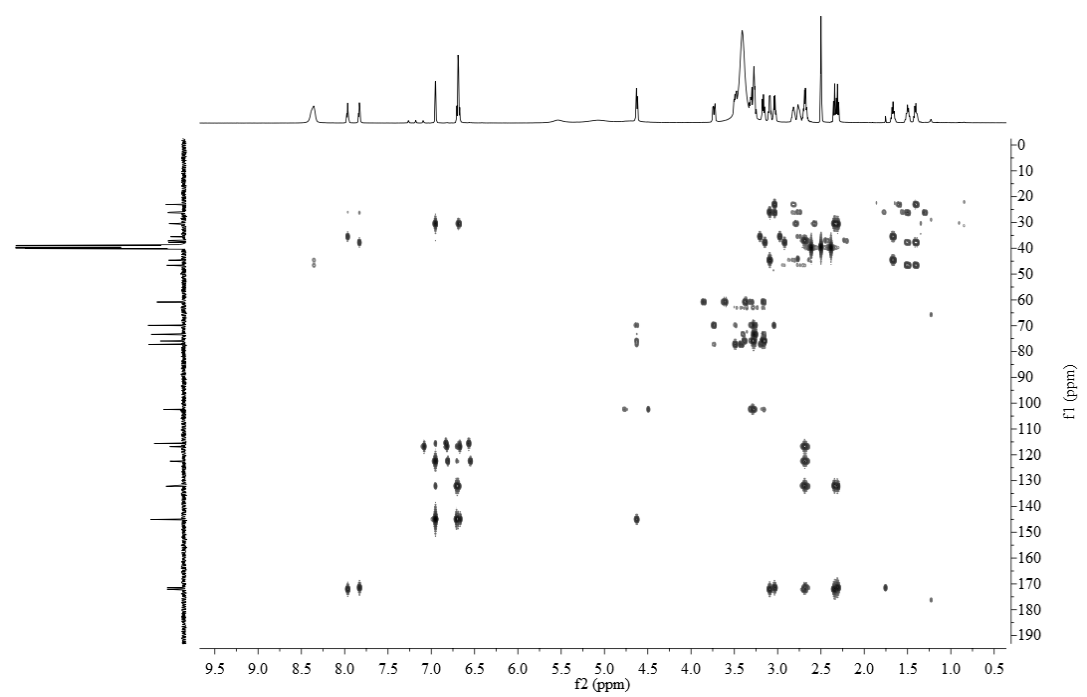

Figure S61. HMBC spectrum of lycibarbarspermidine K (11) in DMSO- $d_{6}$ at $600 \mathrm{MHz}$

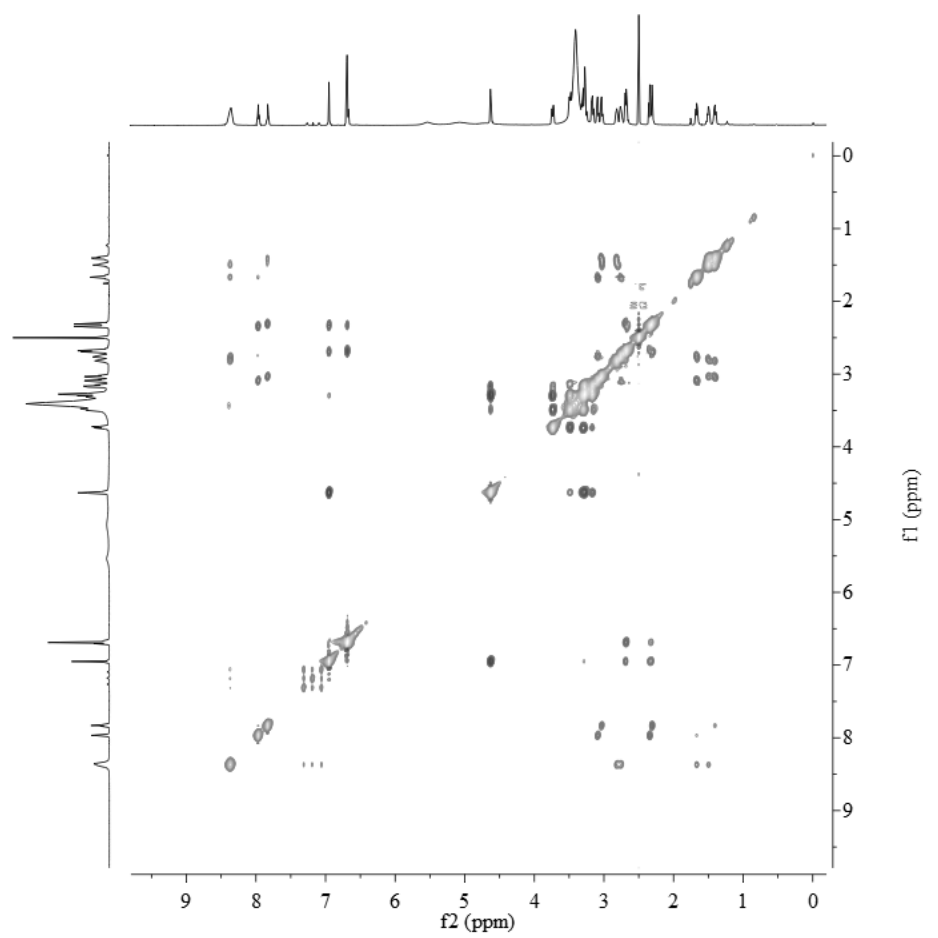

Figure S62. ROESY spectrum of lycibarbarspermidine K (11) in DMSO- $d_{6}$ at $400 \mathrm{MHz}$ 


\section{The 1D and 2D NMR spectra of 12.}

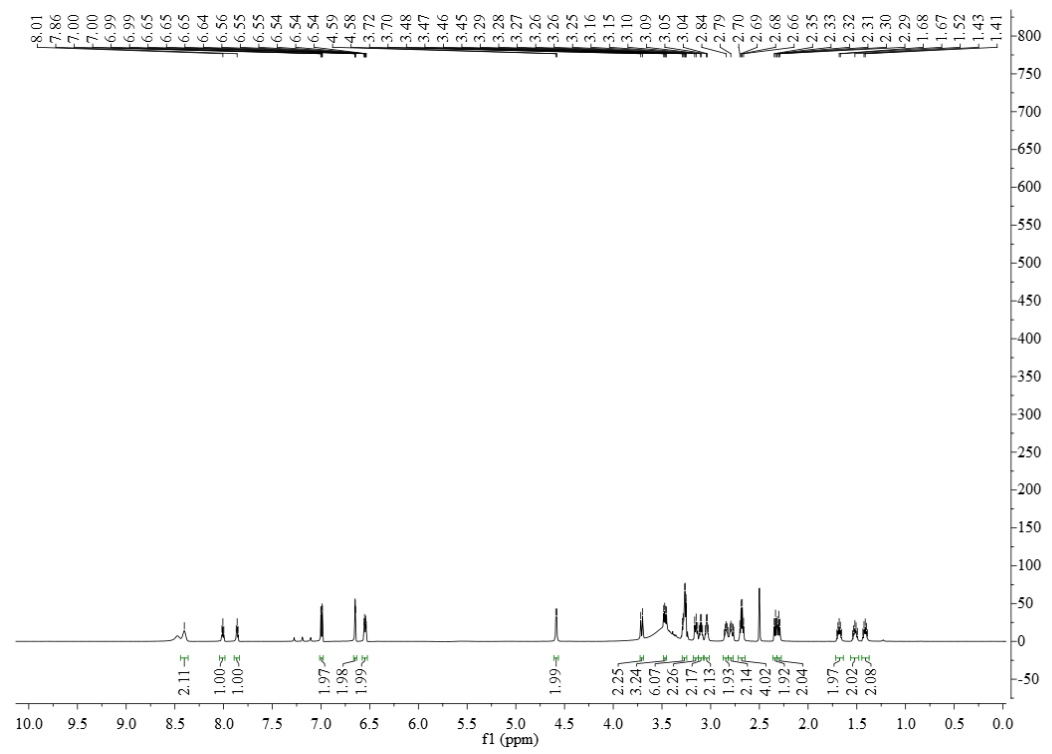

Figure S63. ${ }^{1} \mathrm{H}$ NMR spectrum of lycibarbarspermidine L (12) in DMSO- $d_{6}$ at $600 \mathrm{MHz}$

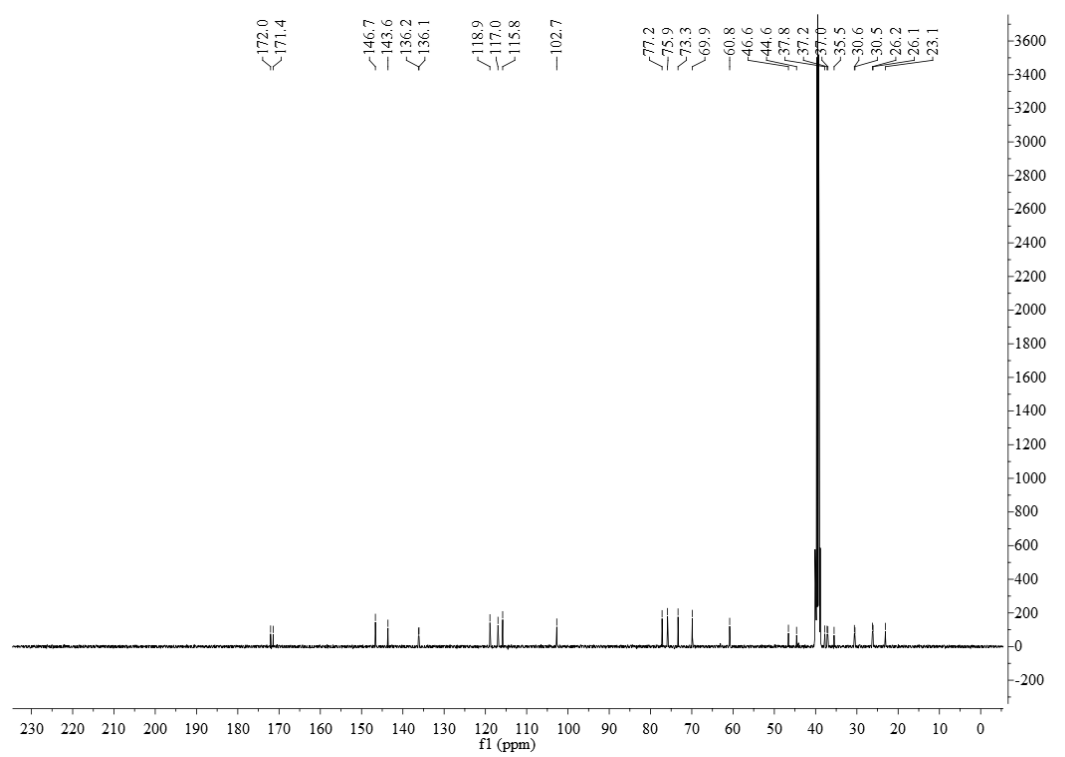

Figure S64. ${ }^{13} \mathrm{C}$ NMR spectrum of lycibarbarspermidine L (12) in DMSO- $d_{6}$ at $100 \mathrm{MHz}$ 


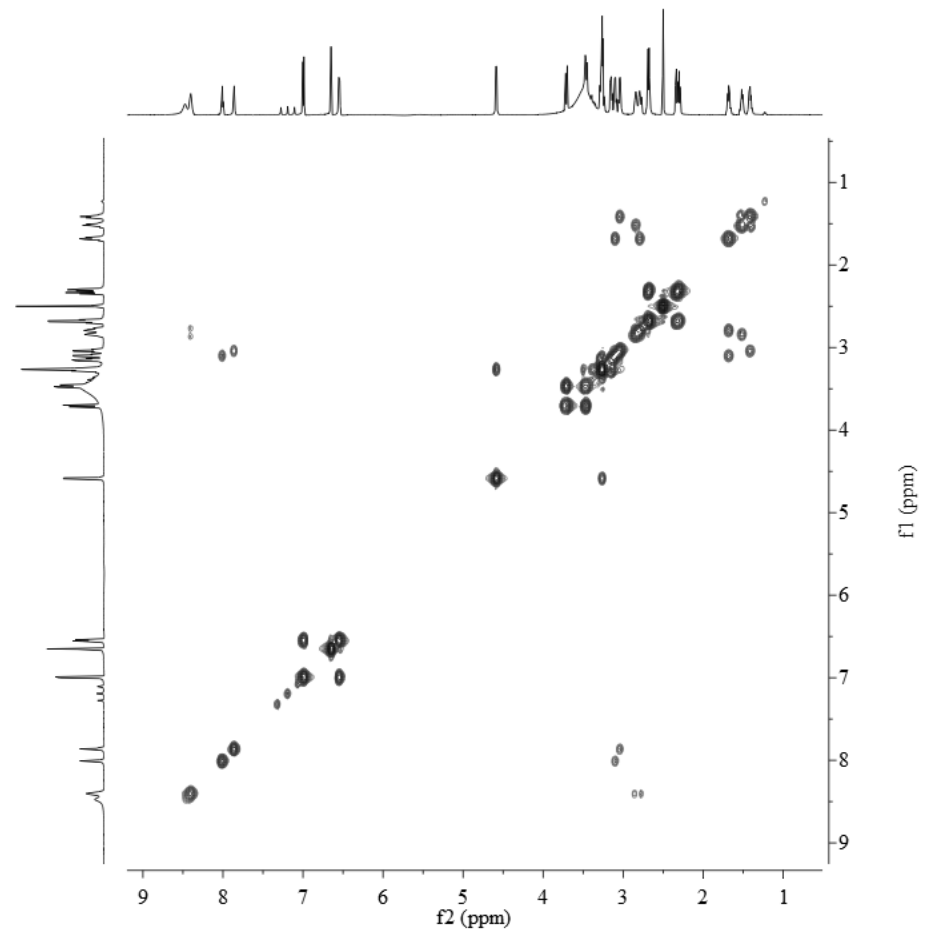

Figure S65. ${ }^{1} \mathrm{H}-{ }^{1} \mathrm{H}$ COSY spectrum of lycibarbarspermidine L (12) in DMSO- $d_{6}$ at $400 \mathrm{MHz}$

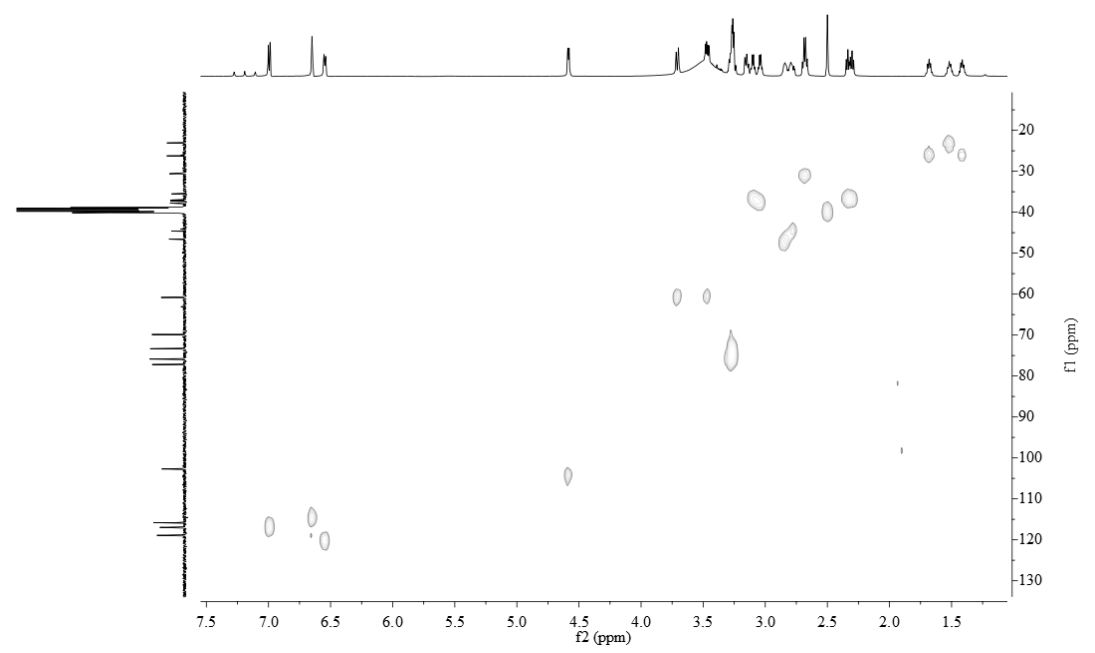

Figure S66. HSQC spectrum of lycibarbarspermidine L (12) in DMSO- $d_{6}$ at $400 \mathrm{MHz}$ 


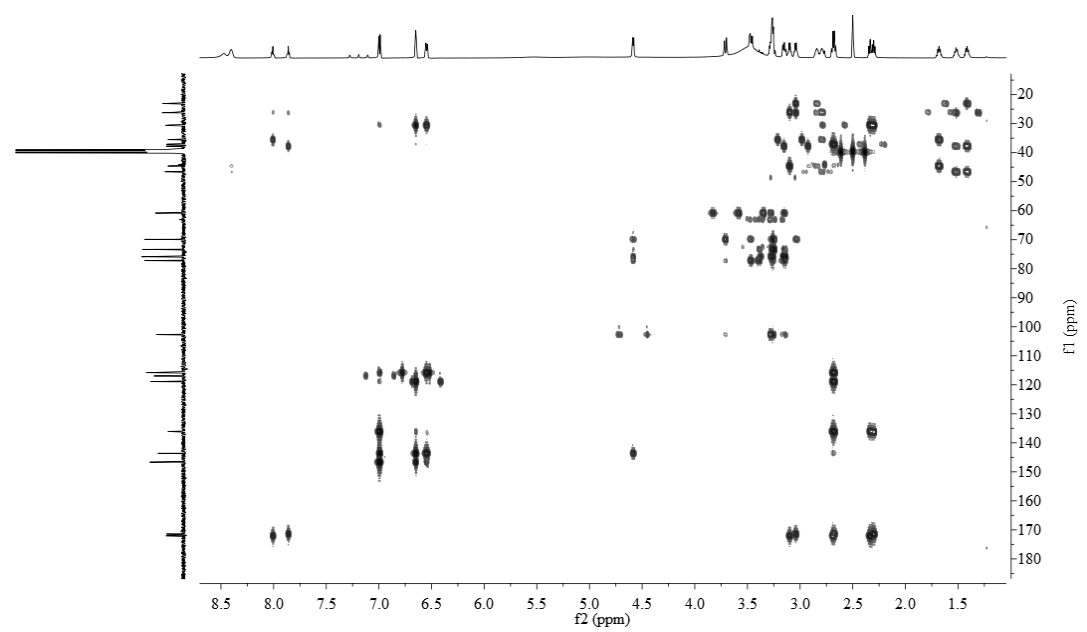

Figure S67. HMBC spectrum of lycibarbarspermidine L (12) in DMSO- $d_{6}$ at $600 \mathrm{MHz}$

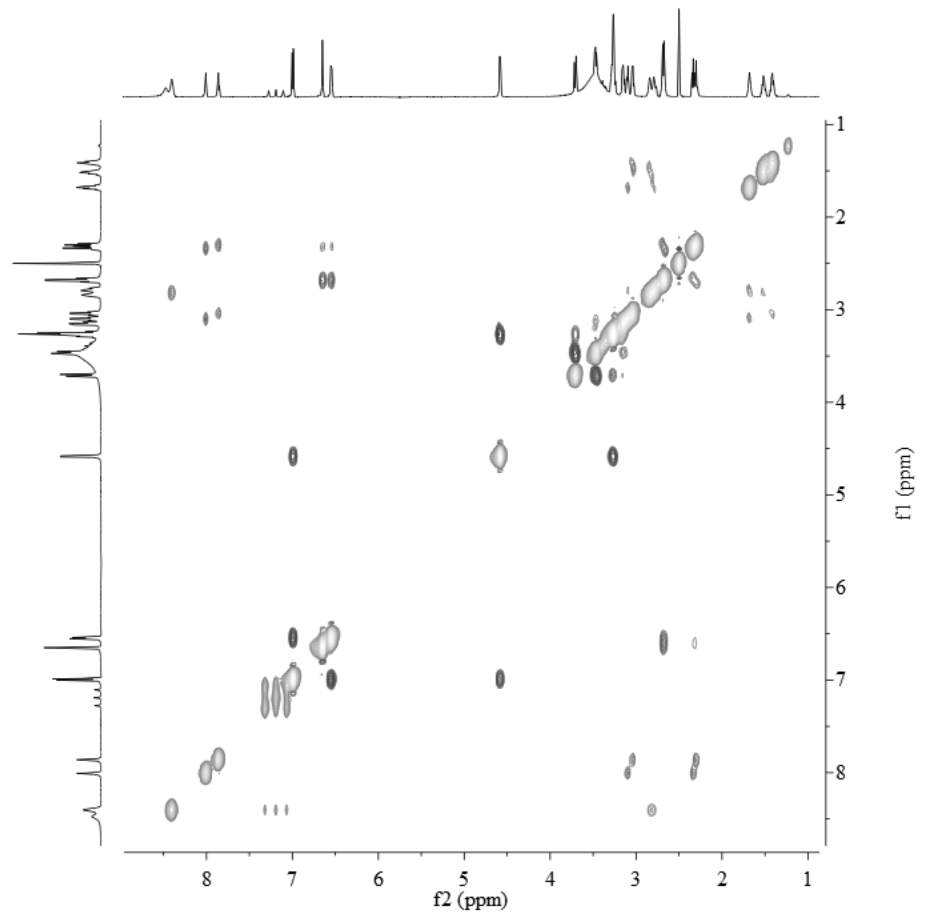

Figure S68. ROESY spectrum of lycibarbarspermidine L (12) in DMSO- $d_{6}$ at $400 \mathrm{MHz}$ 


\section{The 1D and 2D NMR spectra of 13.}

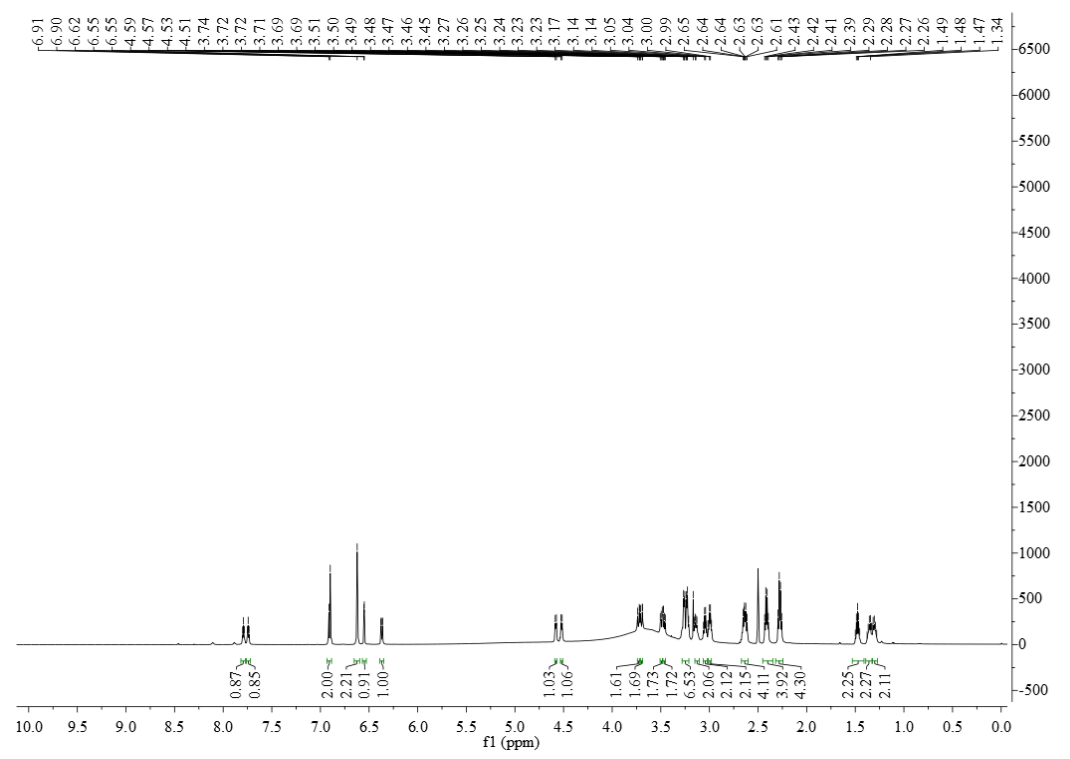

Figure S69. ${ }^{1} \mathrm{H}$ NMR spectrum of lycibarbarspermidine M (13) in DMSO- $d_{6}$ at $600 \mathrm{MHz}$

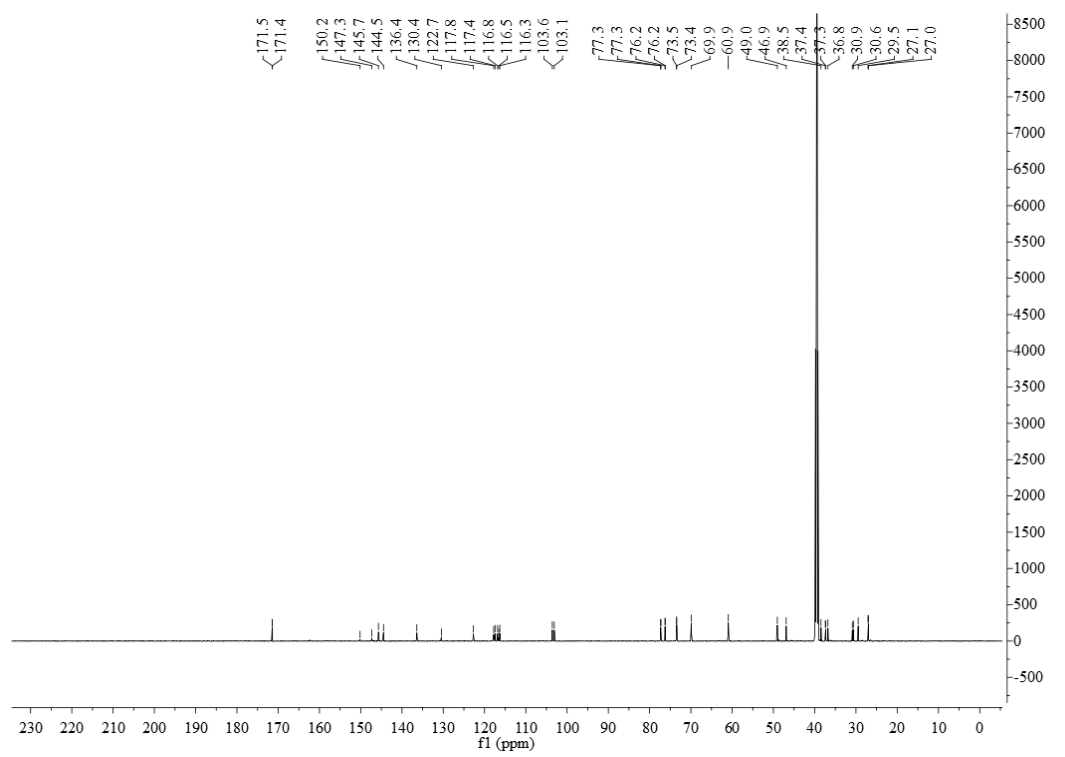

Figure S70. ${ }^{13} \mathrm{C}$ NMR spectrum of lycibarbarspermidine M (13) in DMSO- $d_{6}$ at $150 \mathrm{MHz}$ 


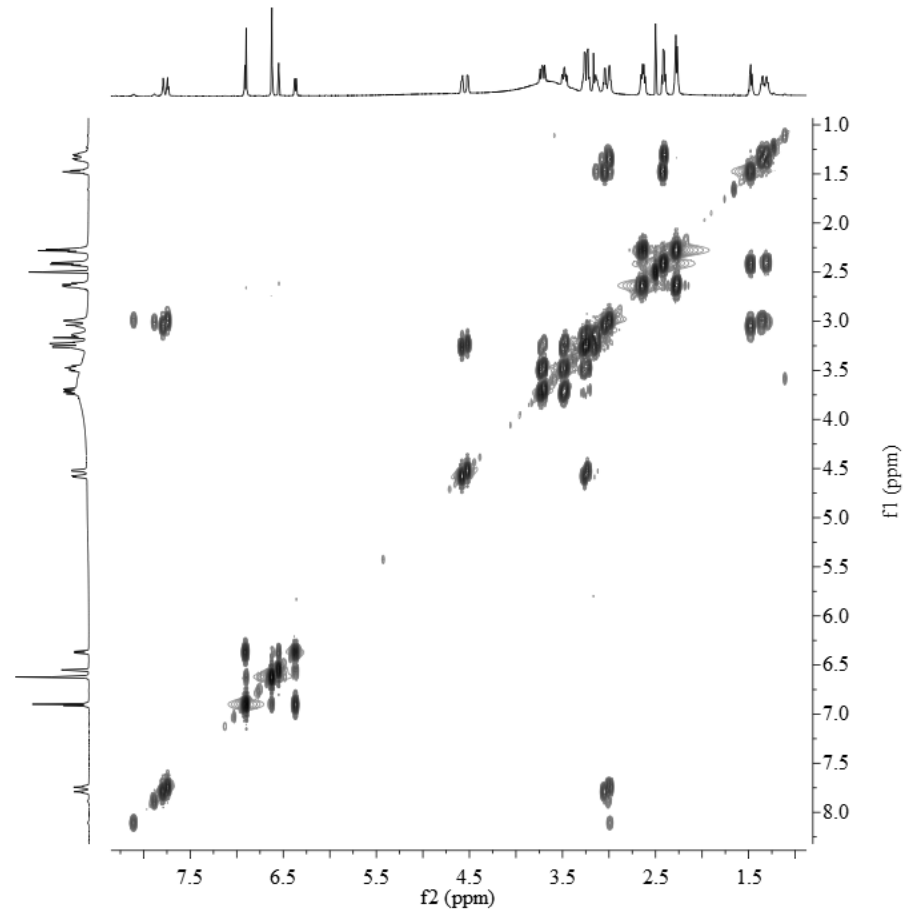

Figure S71. ${ }^{1} \mathrm{H}-{ }^{1} \mathrm{H}$ COSY spectrum of lycibarbarspermidine M (13) in DMSO- $d_{6}$ at $600 \mathrm{MHz}$

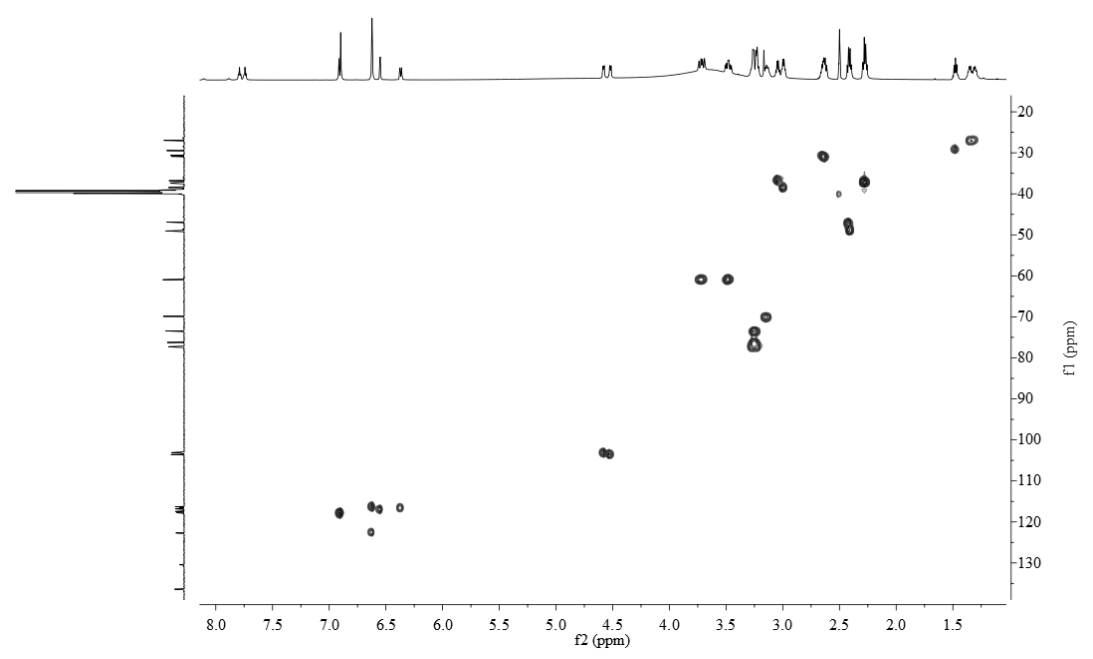

Figure S72. HSQC spectrum of lycibarbarspermidine M (13) in DMSO- $d_{6}$ at $600 \mathrm{MHz}$ 


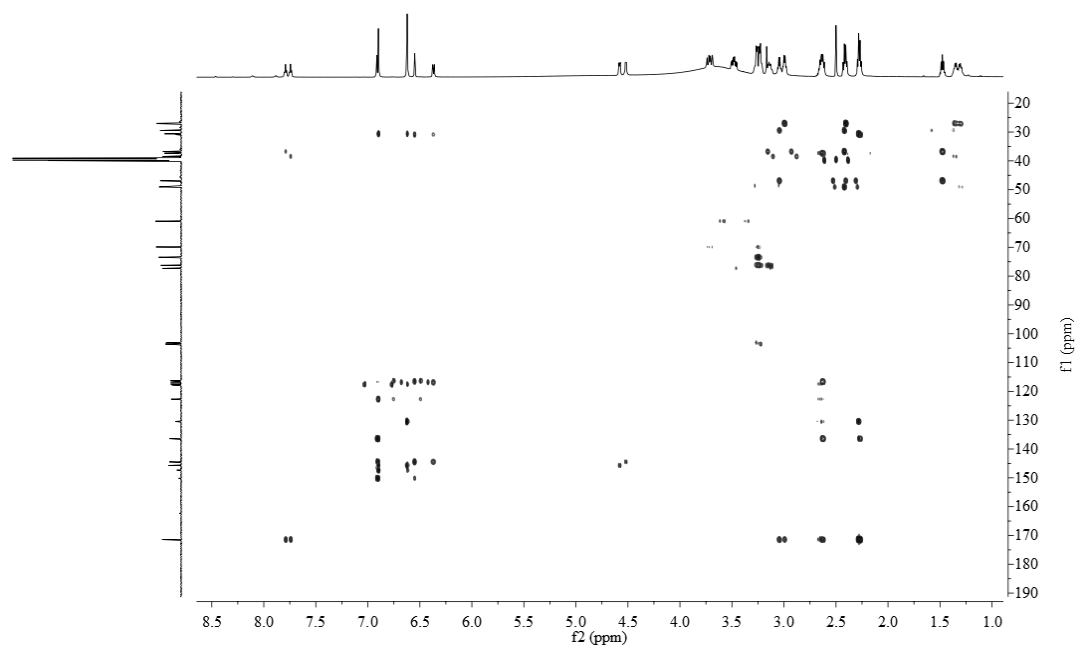

Figure S73. HMBC spectrum of lycibarbarspermidine M (13) in DMSO- $d_{6}$ at $600 \mathrm{MHz}$

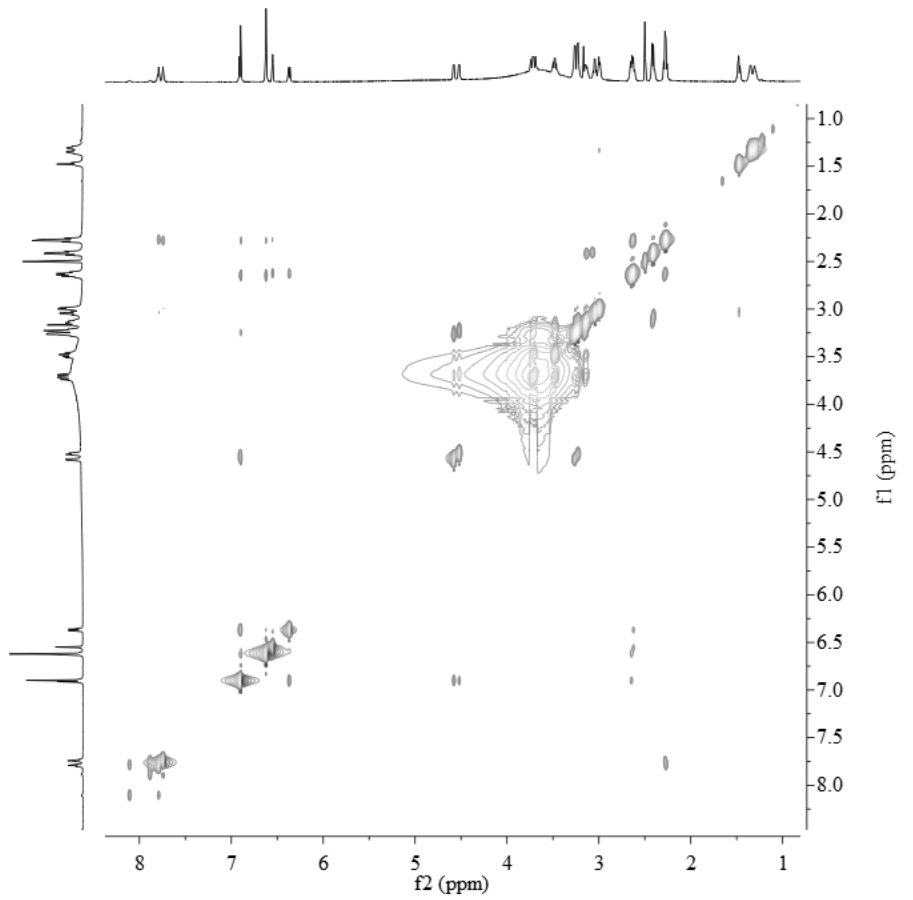

Figure S74. NOESY spectrum of lycibarbarspermidine M (13) in DMSO- $d_{6}$ at $600 \mathrm{MHz}$ 


\section{The 1D and 2D NMR spectra of 14.}

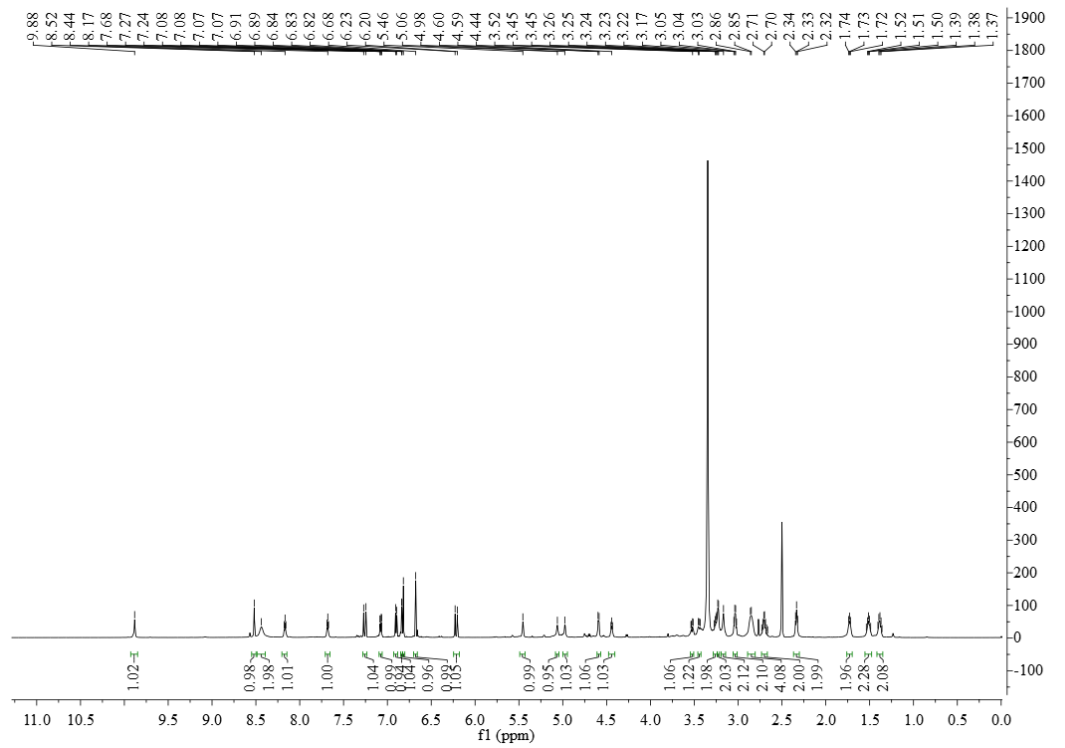

Figure S75. ${ }^{1} \mathrm{H}$ NMR spectrum of lycibarbarspermidine N (14) in DMSO- $d_{6}$ at $600 \mathrm{MHz}$

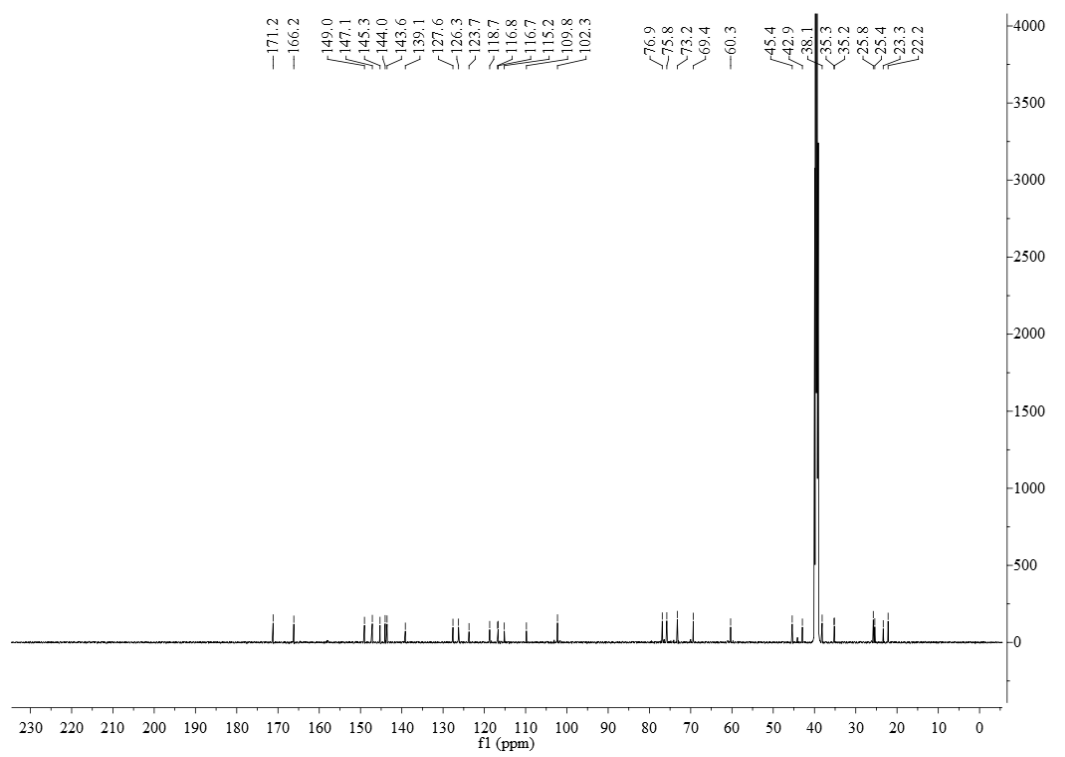

Figure S76. ${ }^{13} \mathrm{C}$ NMR spectrum of lycibarbarspermidine $\mathrm{N}(\mathbf{1 4})$ in DMSO- $d_{6}$ at $150 \mathrm{MHz}$ 


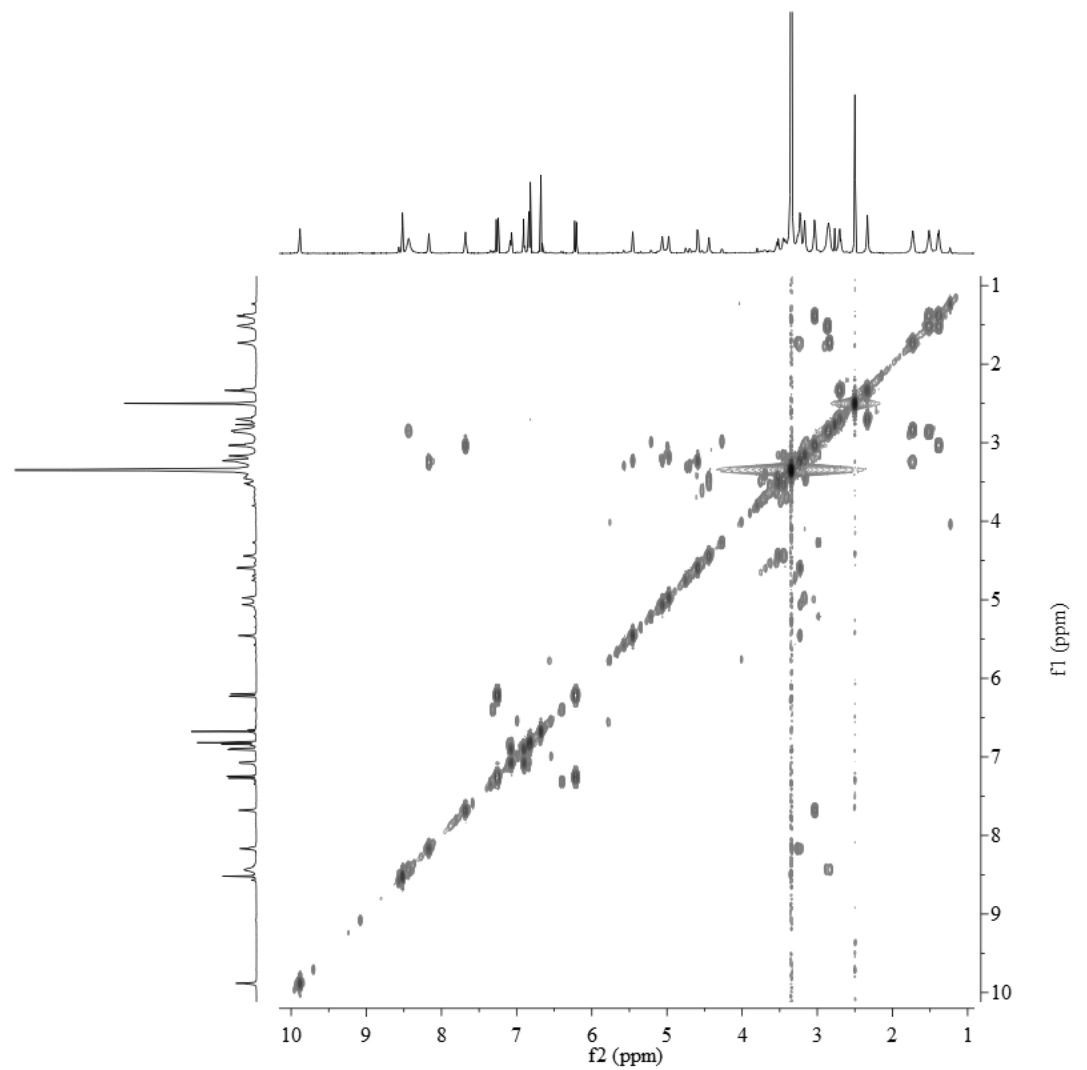

Figure S77. ${ }^{1} \mathrm{H}-{ }^{1} \mathrm{H}$ COSY spectrum of lycibarbarspermidine $\mathrm{N}(\mathbf{1 4})$ in DMSO- $d_{6}$ at $600 \mathrm{MHz}$

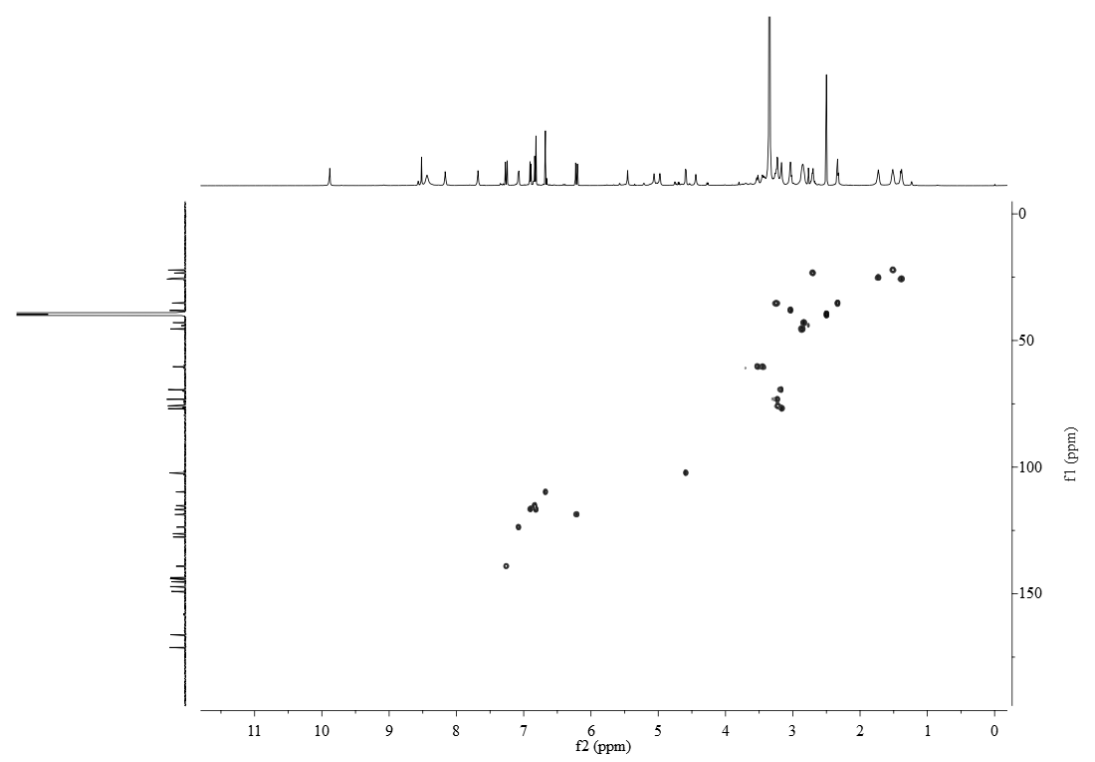

Figure S78. HSQC spectrum of lycibarbarspermidine N (14) in DMSO- $d_{6}$ at $600 \mathrm{MHz}$ 


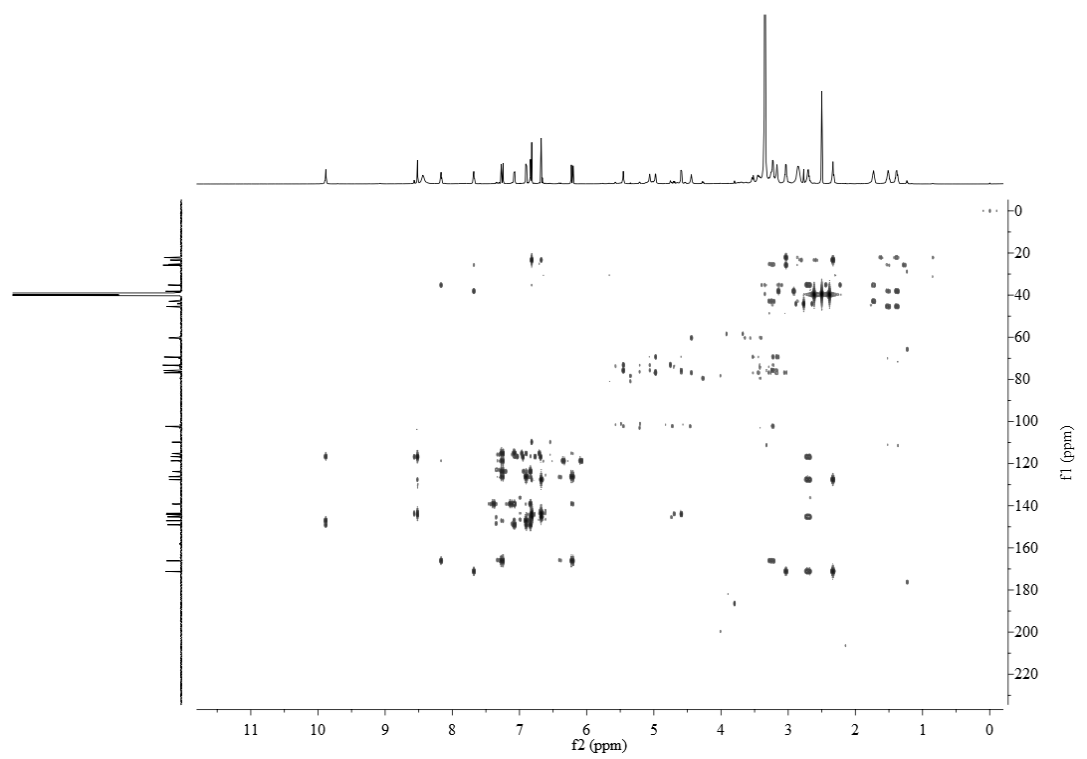

Figure S79. HMBC spectrum of lycibarbarspermidine N (14) in DMSO- $d_{6}$ at $600 \mathrm{MHz}$

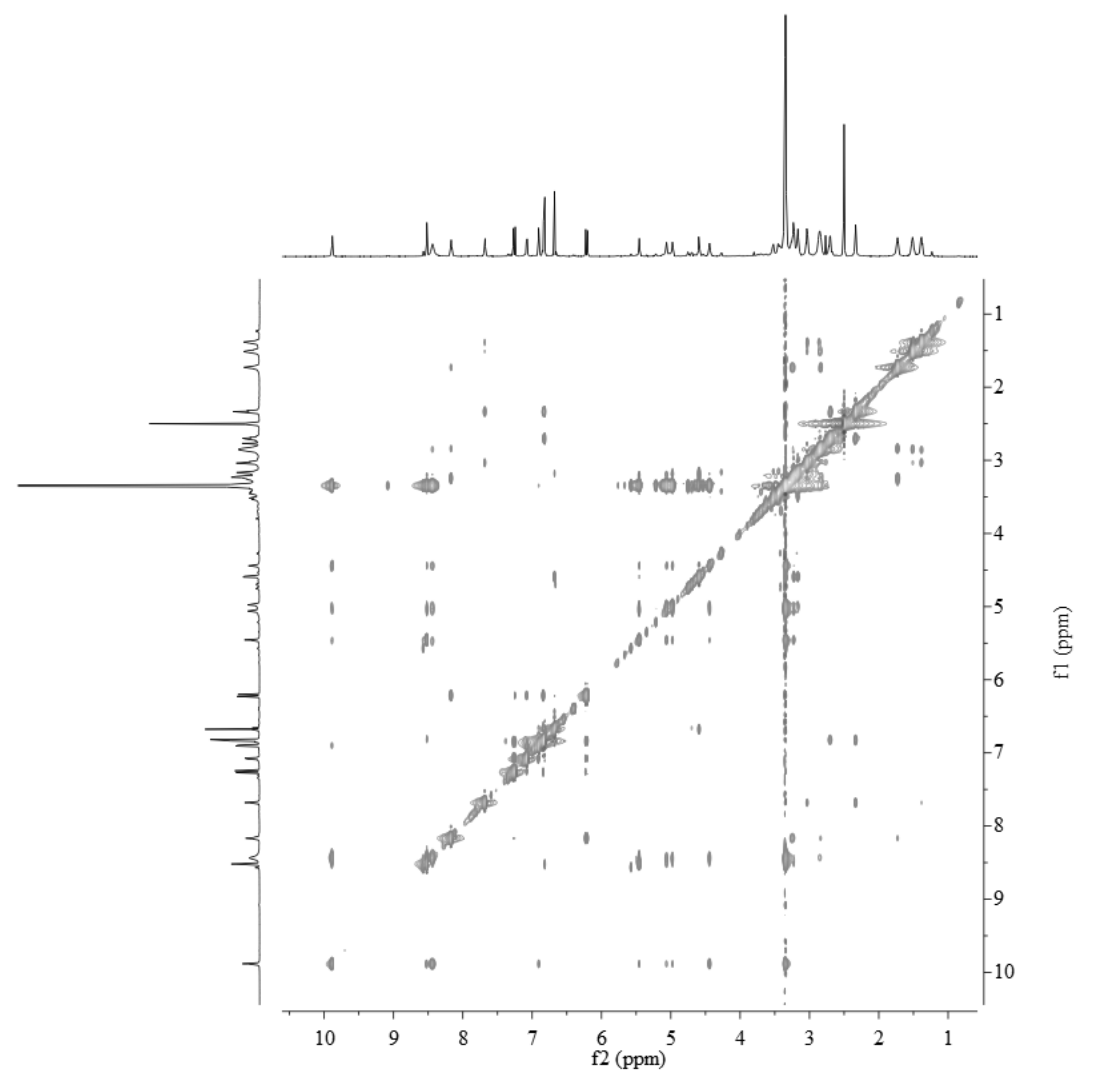

Figure S80. NOESY spectrum of lycibarbarspermidine N (14) in DMSO- $d_{6}$ at $600 \mathrm{MHz}$ 


\section{The 1D and 2D NMR spectra of 15.}

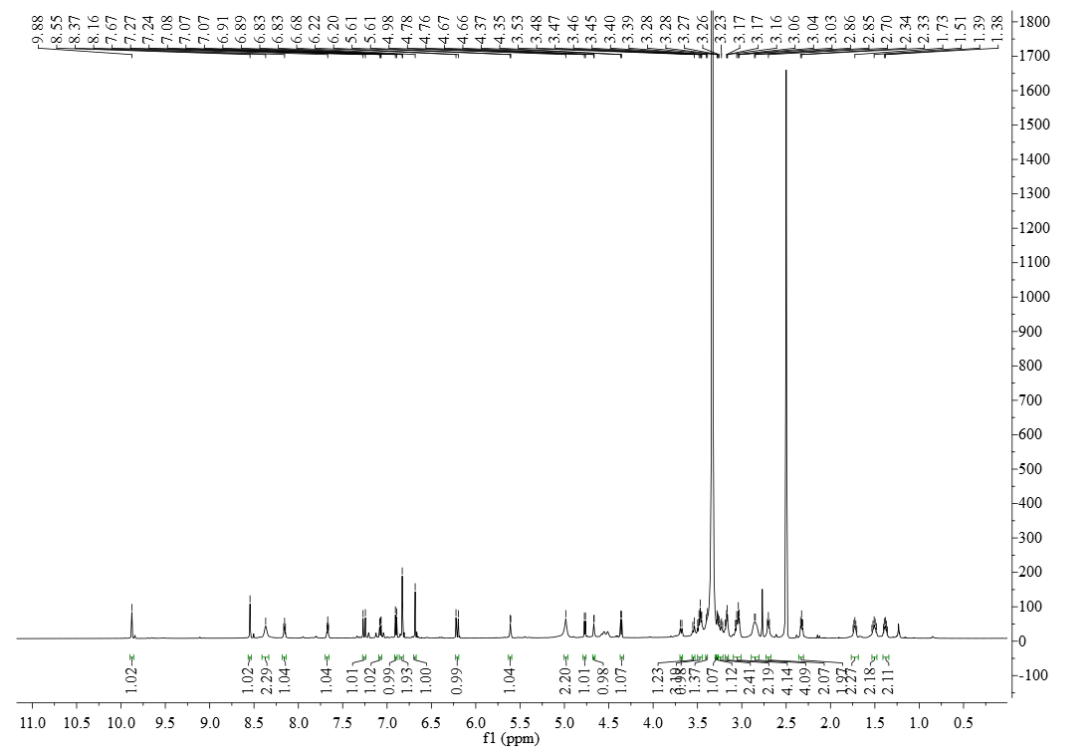

Figure S81. ${ }^{1} \mathrm{H}$ NMR spectrum of lycibarbarspermidine O (15) in DMSO- $d_{6}$ at $600 \mathrm{MHz}$

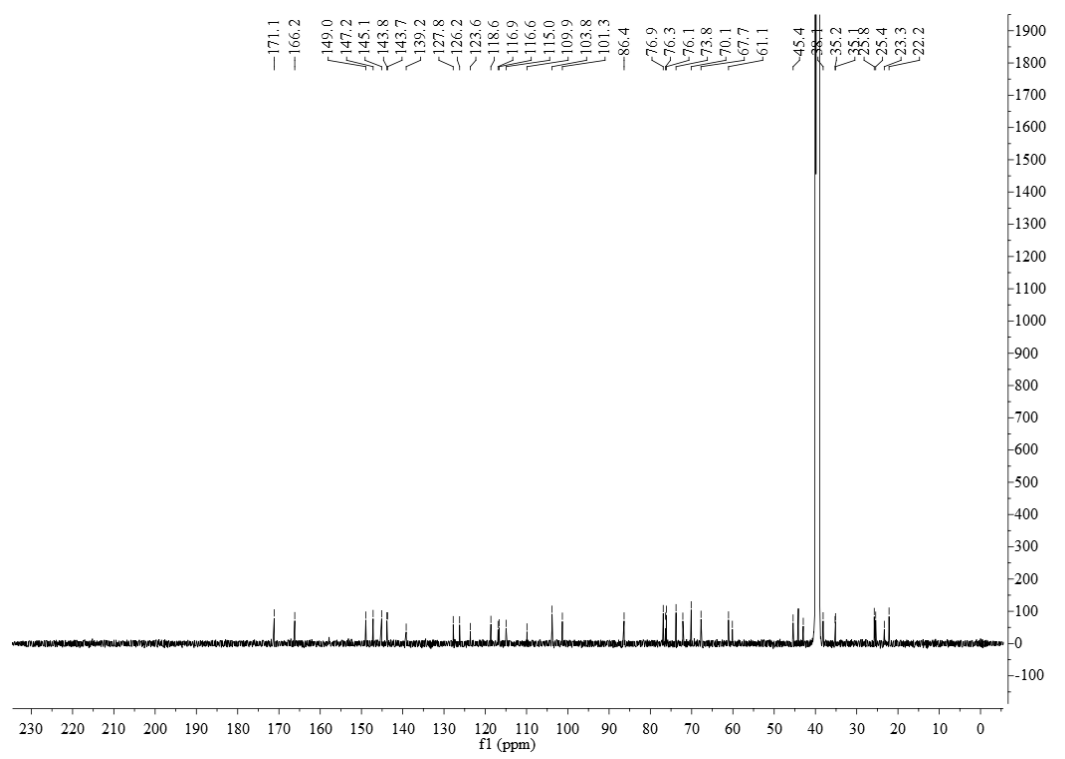

Figure S82. ${ }^{13} \mathrm{C}$ NMR spectrum of lycibarbarspermidine O (15) in DMSO- $d_{6}$ at $150 \mathrm{MHz}$ 


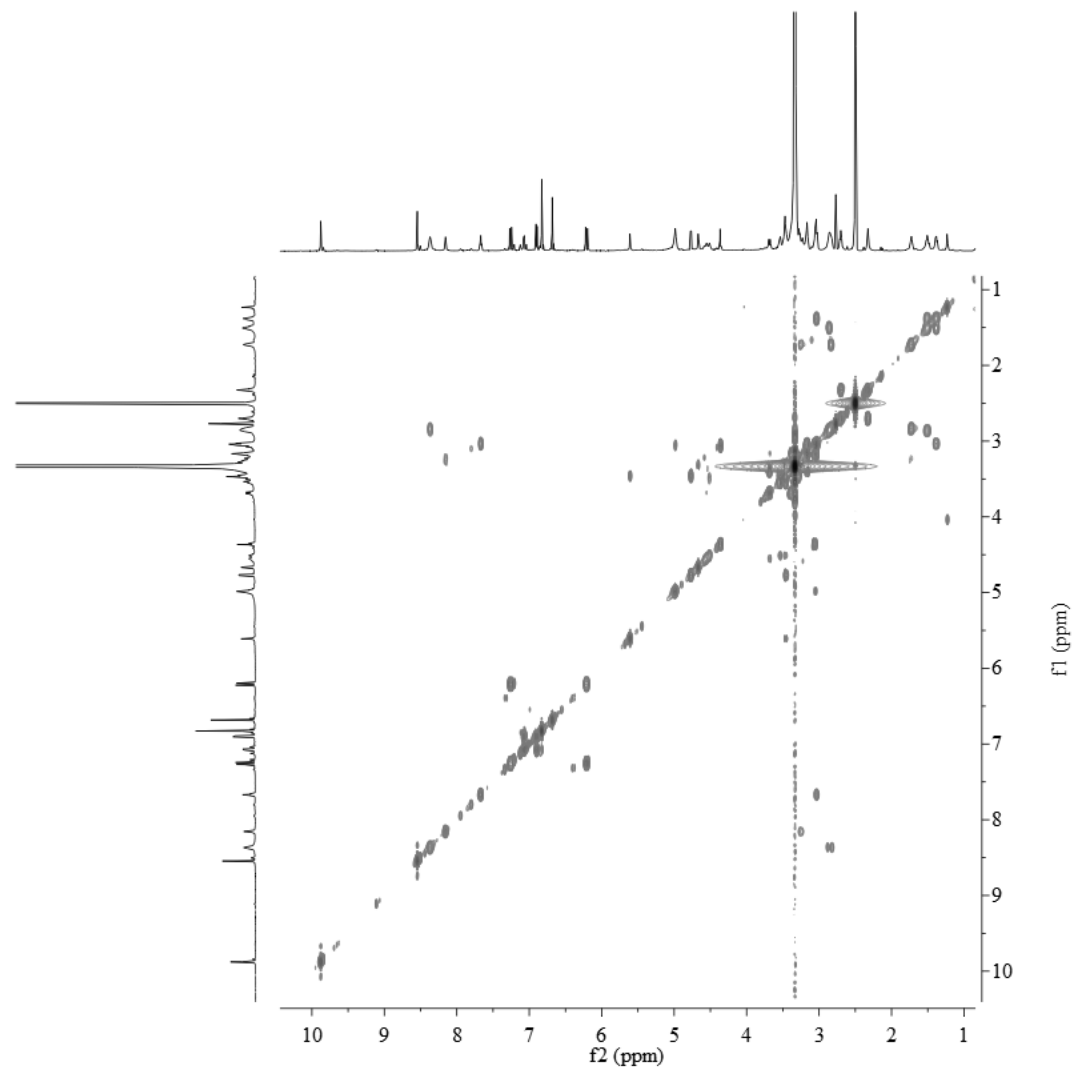

Figure S83. ${ }^{1} \mathrm{H}-{ }^{1} \mathrm{H}$ COSY spectrum of lycibarbarspermidine O (15) in DMSO- $d_{6}$ at $600 \mathrm{MHz}$

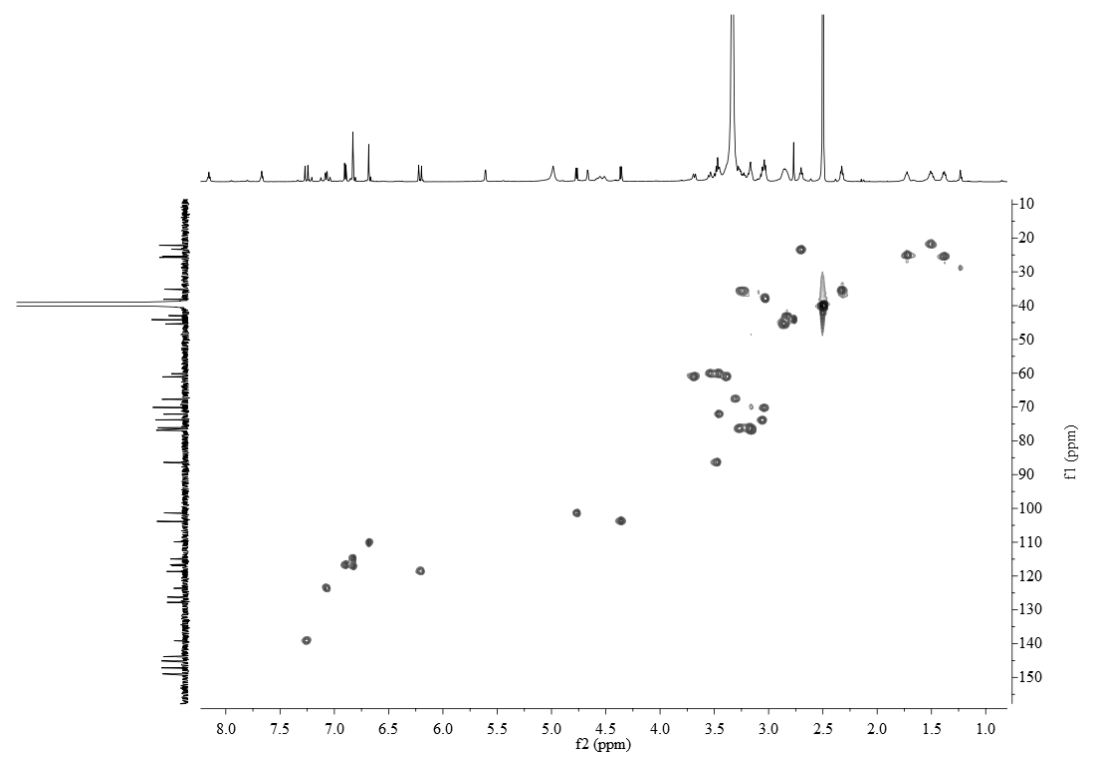

Figure S84. HSQC spectrum of lycibarbarspermidine O (15) in DMSO- $d_{6}$ at $600 \mathrm{MHz}$ 


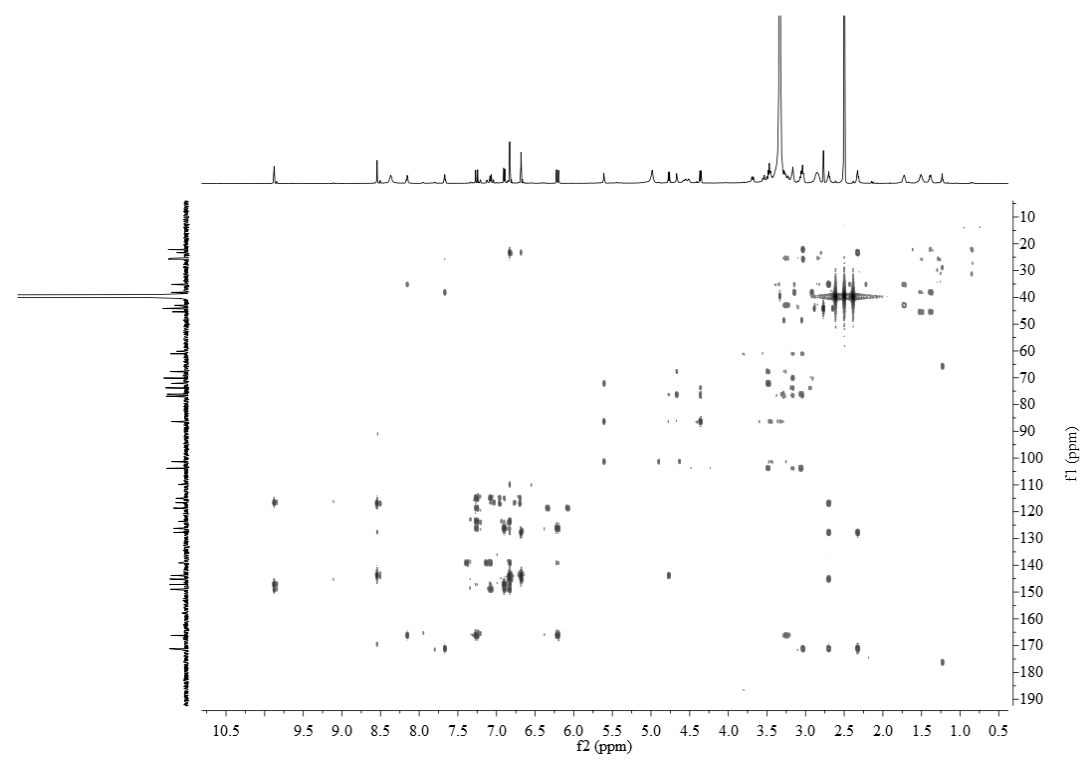

Figure S85. HMBC spectrum of lycibarbarspermidine O (15) in DMSO- $d_{6}$ at $600 \mathrm{MHz}$

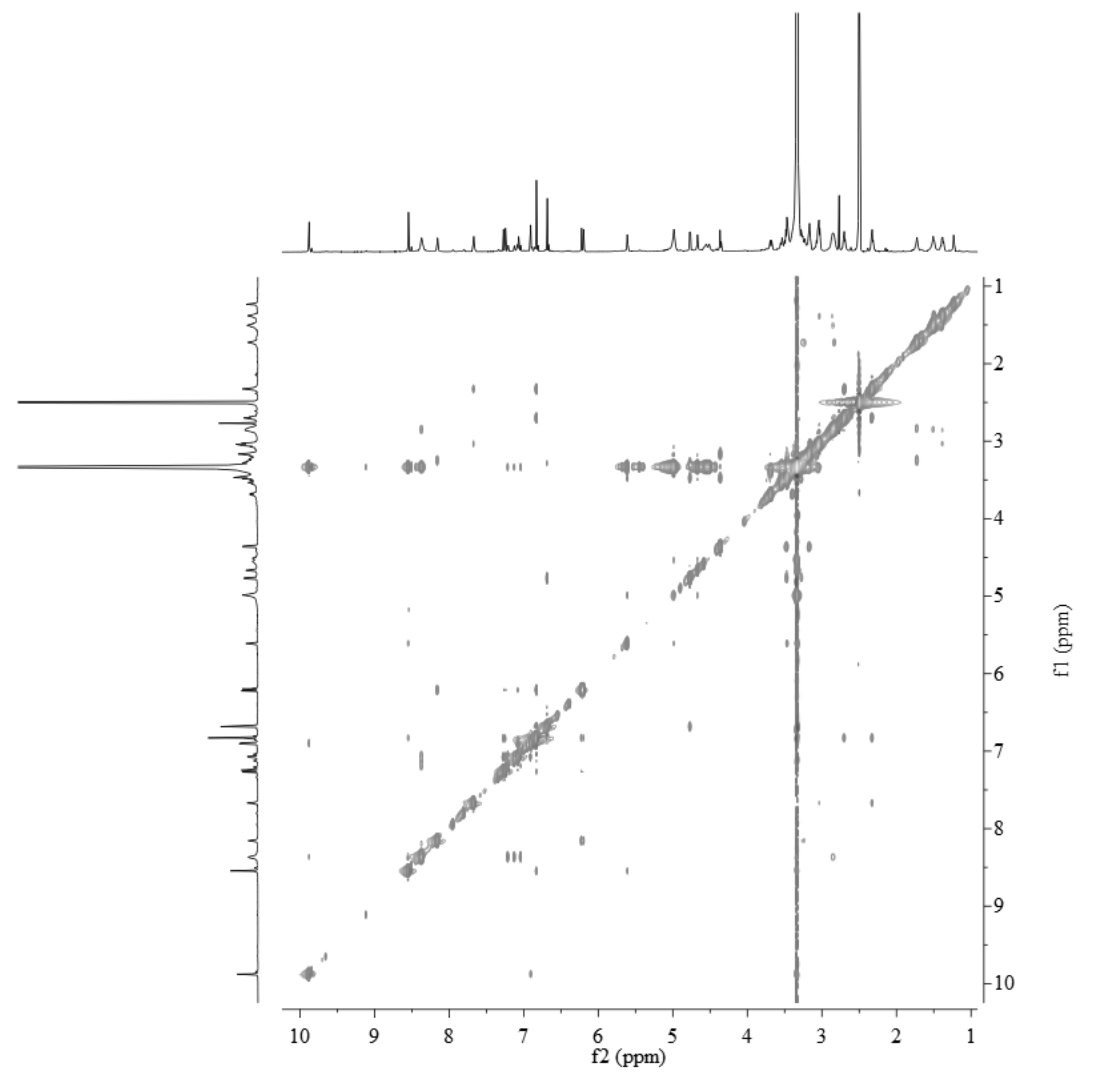

Figure S86. NOESY spectrum of lycibarbarspermidine O (15) in DMSO- $d_{6}$ at $600 \mathrm{MHz}$ 